\title{
INNER-SHELL PHOTOEMISSION FROM ATOMS AND MOLECULES USING SYNCHROTRON RADIATION
}

\author{
Dennis William Lindle \\ (Ph.D. Thesis)
}

\author{
Materials and Molecular Research Division \\ Lawrence Berkeley Laboratory \\ and \\ Department of Chemistry \\ University of California \\ Berkeley, California 94720
}

\section{DISCLAIMER}

\begin{abstract}
This report was prepared as an account of work sponsored by an agency of the United States Government Neither the United States Government nor any agency thereof, nor any of their employees, makes any warranty, express or implied, or assumes any legal liability or responsibility for the accuracy, completeness, or usefulness of any information, apparatus, product, or process disclosed, or represents that its use would not infringe privately owned rights. Reference herein to any speciffe commercial product, process, or service by trade name, trademark, manufacturer, or otherwise does not necessarily constitute or imply its endorsement, recommendation, or favoring by the United States Government or any agency thereof. The views and opinious of authors expresed berein do not necessarily state or reflect those of the United States Govemment or any agency thereof.
\end{abstract}

This work was supported by the Director, Office of Energy Research, Office of Basic Energy Sciences, Chemical Sciences Division of the U.S. Department of Energy under Contract NO. OE-ACO3-76SF00098. It was performed at the Stanford Synchrotron Radiation Laboratory, which is supported by the Department of Energy, Office of Basic Energy Sciences and the National Science Foundation, Division of Materials Research. 


\title{
INNER-SHELL PHOTOEMISSION FROM ATOMS AND MOLECULES USING SYHCHROTRON RADIATION
}

\author{
Dennis William Lindle \\ Materials and Molecular Research Division \\ Lawrence Berkeley Laboratory \\ and \\ Department of Chemistry \\ University of California \\ Berkeley, California 94720
}

Abstract

Photoelectron spectroscopy, in conjunction with synchrotron radiation, has been used to study inner-shell photoemission from atoms and molecules. The time structure of the synchrotron radiation permits the measurement of time-of-flight (TOF) spectra of Auger and photoelectrons, thereby increasing the electron collection efficiency. The double-angle TOF method yielded angle-resolved photoelectron intensities, which were used to determine photoionization cross sections and photoelectron angular distributions in several cases. Comparison to theoretical calculations has been made where possible to help explain observed phenomena in terms of the electronic structure and photoionization dynamics of the systems studied.

Measurements have been made for the $3 d$ subshell in krypton, the iodine $4 \mathrm{~d}$ and $4 \mathrm{p}$ subshells in methyl iodide, the nitrogen $K$ shell in $\mathrm{N}_{2}$ and $\mathrm{NO}$, and the main and satellite lines in helium. For the $\mathrm{Kr}$ 3d subshe11, angular-distribution asymmetry parameters are presented for photon energies from 100 to $600 \mathrm{eV}$ which show large changes 
attributable to important one-electron effects. The asymetry parameter fell below the Hartree-Fock-theory prediction at high photon energy, and showed resonant interchannel-coupling effects near the $3 p$ threshold. The total intensity of shake-up satellites relative to the $\mathrm{Kr} 3 \mathrm{~d}$ main line decreased with photon energy in the range 180 to 270 eV, and the average asymmetry parameter for these satellite states showed a marked increase over the same energy range.

For methyl iodide, the first photoemission measurements on the I 4d and I 4p subshells are presented. Cross sections and asymmetry parameters were measured from threshold to $300 \mathrm{eV}$ photon energy for the I 4d level (to $440 \mathrm{eV}$ for the asymmetry parameter), and from 175 to $300 \mathrm{eV}$ for the I $4 \mathrm{p}$ level. The I $4 \mathrm{~d}$ results exhibited atomic-like behavior throughout this energy range, mimicking similar behavior for the $X e \mathrm{~d}$ subshell and indicating that the $I 4 d$ subshell is localized on the iodine atom in $\mathrm{CH}_{3} \mathrm{I}$. Near threshold, the spin-orbit final states, $4 d_{5 / 2}$ and $4 d_{3 / 2}$, were resolved and exhibited non-statistical intensity ratios mainly due to a kinetic-energy effect. The I $4 p$ asymetry-parameter results were essentially identical to the asymmetry-parameter results for the I $4 \mathrm{~d}$ subshell at the same photon energy, suggesting strong interchannel coupling, despite the fact that the I $4 p$ cross section is of the same order as the I $4 d$ cross section in the energy range measured.

Also reported are the first gas-phase photoemission measurements near the nitrogen $K$ edges of $N_{2}$ and $N_{0}$. Shape-resonance behavior was observed in the cross sections for both $N$ is photoemission and $N$ 
v

KVV Auger emission. The asymmetry parameters for $N$ is photoemission show larger contrast ratios than the cross-section data, and agree with multiple-scattering calculations except for a small energy shift. In He, the partial cross section, the satellite branching ratio, and the asymmetry parameter for simultaneous photoionization and excitation to the $\mathrm{n}=2$ states of the $\mathrm{He}^{+}$ion have been measured in the energy range directly above threshold. The asymnetry-parameter results have been used to determine the ratio of the cross sections for producing the $2 p$ final state versus the $2 s$ final state; the $\mathrm{He}^{+}(n=2)$ satellite was shown to be predominantly $2 p$ near threshold, in agreement with the experimental and most of the theoretical results reported to date. In the region below the $\mathrm{He}^{+}(n=3)$ threshold at 73 $\mathrm{eV}$, the effects of a series of autoionizing Rydberg levels on the cross sections and asymmetry parameters for the $n=2$ satellites were observed, this being the first detailed measurement of the angular distribution of a satellite over an autoionization resonance. Parameters defining all of the measured resonance profiles were obtained, and in particular, some individual dipole matrix elements were derived from the experimental results.

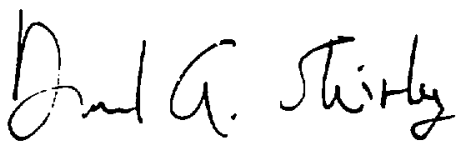


Table of Contents

Page

ABSTRACT. ...............................

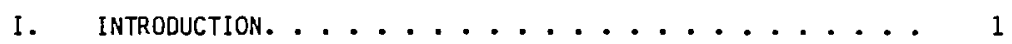

References.................. g

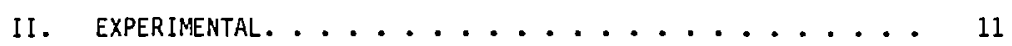

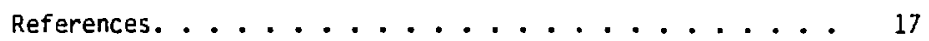

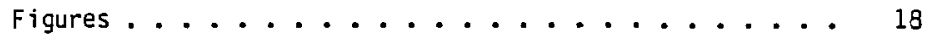

III. PHOTOEMISSION FROM THE $3 d$ SUBSHELL OF ATOMIC $\mathrm{kr} \ldots . . . .22$

References................. 27

figures . . . . . . . . . . . . . . . . . 29

IV. INNER-SHELL PHOTOEMISSION FROM THE IODINE ATOM IN $\mathrm{CH}_{3} \mathrm{I} \ldots 33$

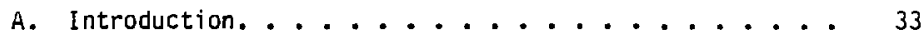

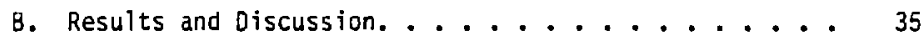

1. I 4d Subshell ............... 35

2. I ' $4 p$ ' Subshell ............. 4 42

C. Conclusions ................... 44

References. ................. 45

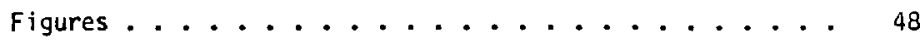

v. NITROGEN K-SHELL PHOTOEMISSION AND AUGER EMISSION

FROM $N_{2}$ AND NO. ...................... 55

References...................... 63

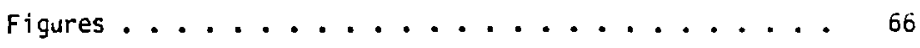


VI. PHOTOIONIZATION OF HELIUM ABOVE THE He ${ }^{+}(n=2)$ THRESHOLD:

AUTOIONIZATION AND FINAL-STATE SYMMETRY . . . . . . . . 71

A. Introduction. .................. 71

B. Nonresonance Photoionization. . . . . . . . . 74

C. Resonance Photoionization . . . . . . . . . 81

1. Theoretical Background. . . . . . . . . . 82

2. Data Analysis ............... 89

3. Results - Cross Sections and Branching Ratios ... 93

4. Results - Asymmetry Parameters. . . . . . . 101

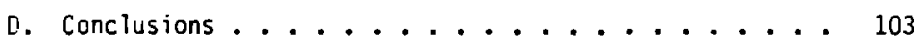

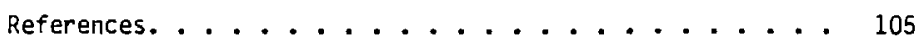

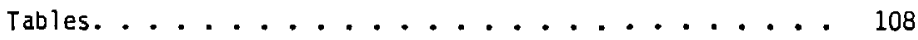

Figures ..................... 115

ACKNOWLEGDEMENTS. . . . . . . . . . . . . . . 126 


\section{INTRODUCTION}

Photoelectron spectroscopy, in which electrons ionized by photon impact are studied, is an excellent tool for determining the electronic properties (structure and dynamics) of atoms and molecules. The directness of this technique for studying electronic structure is illustrated by the very simple idea, first proposed by Koopmans, 1 that the ionization potential, $I_{j}$, of an electron in a bound orbital, $j$, is equal in magnitude to the one-electron orbital energy, $E_{j}$,

$$
I_{j}=-E_{j} \text {. }
$$

This good first approximation provides that the measurement of $I_{j}$ 's can yield a direct representation of the orbital energy diagram of an atom or molecule. Koopmans' theorem is especially helpful for studies that attempt to determine electronic structure only. ${ }^{2,3}$ The understanding of the dynamics of the photoionization process, however, necessitates going beyond Koopmans' theorem and electronic-structure measurements, and generally requires more sophisticated experimental and theoretical techniques. The majority of the work along these lines has been concerned with electrons in valence orbitals. ${ }^{4}$ This dissertation presents results of experimental studies of photoionization dynamics in an area in which relatively little work has been done; inner-shell photoemission from atoms ( $\mathrm{Kr}$ and $\mathrm{He}$ ) and molecules $\left(\mathrm{CH}_{3} \mathrm{I}, \mathrm{N}_{2}\right.$, and $\left.\mathrm{NO}\right)$.

A photoelectron spectrum is a plot of the number of electrons 
emitted by photoionization as a function of their kinetic energy, $\varepsilon_{j}$. For a given photon energy, $h_{v}$, the photoelectron spectrum is characterized by a discrete set of photoemission peaks as a consequence of the energy-conservation expression

$$
\varepsilon_{j}=h_{v}-I_{j} \cdot
$$

Recourse to Eq. (1) and an appropriate theoretical energy-level calculation then may yield an association of each peak in the spectrum with a particular final state of the resulting ion. This constitutes a determination of electronic structure. More information may be gained by taking photoelectron spectra at many different photon energies. For example, peak intensities as a function of photon energy are proportional to the photoionization cross section. Neglecting ali but electric-dipole interactions, ${ }^{5}$ the cross section, $\sigma_{j}(\varepsilon)$, for ionizing an electron from orbital $j$ (or equivalently, for producing the ionic state $j$, where the ion has lost. an electron from orbital j) is given by ${ }^{6}$

$$
\sigma_{j}(c)=\frac{4 \pi^{2} a a_{0}^{2}}{3}\left(\varepsilon_{j}+I_{j}\right) \sum_{k} \mid\left\langle\left.\omega_{j}(N-1)_{\phi_{k}}(c)\left|\sum_{\mu=1}^{N} \vec{r}_{\mu}\right| \psi_{i}(N)\right|^{2},\right.
$$

where $a$ is the fine-structure constant, $a_{0}$ is the Bohr radius, $(\mathrm{N}-1)$ is the $(N-1)$-electron wavefunction of the $i o n$, the $\phi_{k}$ are single-electron wavefunctions of the possible continuum states $k$ of the photoelectron, $\vec{r}_{\mu}$ is the dipole operator, where $\mu$ represents 
the coordinates of each electron in the ground state, $i$, and $\psi_{i}^{(N)}$ is the $\mathrm{N}$-electron ground-state wavefunction of the neutral atom or molecule. Equation (3) shows that the cross section is an incoherent sum of dipole matrix elements between the initial ground state and the final ion-continuum electron states. Dependence of the cross section on the photon energy is included both explicitly, in the term $r_{j}+I_{j}(=h v)$, and implicitly in the energy-dependent functions $p_{k}(\varepsilon)$ within the matrix elements. It is this feature, the photonenergy dependence of the cross section (and other parameters), that reveals the dynamics of the photoionization process.

Aside from the photoionization cross section, each photoelectron peak can be characterized by its spatial or angular distribution. For randomly oriented gas-phase atoms or molecules ionized by linearly polarized radiation, Yang's theorem yields 7

$$
\frac{d \sigma_{j}(\varepsilon)}{d \Omega}=\frac{\sigma_{j}(\varepsilon)}{4 \pi}\left[1+\beta_{j}(\varepsilon) P_{2}(\cos \theta)\right],
$$

where $d o_{j}(\varepsilon) / d \Omega$ is the differential cross section in the solid angle $\Omega, \theta$ is the angle between the photoelectron propagation vector and the polarization vector of the ionizing radiation, $P_{2}(\cos \theta)$ is the second Legendre polynomial $\left[1 / 2\left(3 \cos ^{2} \theta-1\right)\right]$, and $\beta_{j}(\varepsilon)$ is the angular-distribution asymmetry parameter. Within the dipole approximation, the asymmetry parameter completeiy describes the angular distribution of photoelectrons. The requirement that the differential cross section remain non-negative restricts $B_{j}(\varepsilon)$ to the range -1 to 
2. Because $B_{j}(\varepsilon)$ is not an angle-integrated quantity, it may depend on the coherent sum of dipole matrix elements, in contrast to $\sigma_{j}(\varepsilon)$. Thus, the measurerrent of asymetry parameters yields complementary information to cross-section data, and generally the asymmetryparameter results show more dramatic energy-dependent changes as a consequence of the phase interference introduced by the coherent summing of matrix elements.

The goal of this work has been the study of photon-energy dependent behavior of cross sections and asymmetry parameters for inner shells of atoms and molecules in order learn more about single-electron, resonant, and multi-electron effects in photoionization. The remainder of this chapter will discuss briefly the different types of effects that influence the results presented in Chapters III-VI. For convenience, a distinction is made between single-electron and multi-electron processes. The former are of interest to an analysis of photoionization dynamics; the latter to the understanding of electron-correlation phenomena.

The single-electron approximation assumes that the initial ground state of an atom or molecule can be described adequately by a product of one-electron orbital wavefunctions, and that photoionization corresponds to removal of an electron from one of these orbitals. From Eq. (3), this implies that the cross section is dependent on a set of one-electron matrix elements, which can be approximated as modified overlap matrix elements between the initial and continuum wavefunctions. This latter assumption provides a particularly simple 
picture within which to understand the energy dependence of cross sections and asymmetry parameters. For example, photoionization of $\mathrm{Kr}$ $3 \mathrm{~d}$ electrons to ef photoelectrons, ${ }^{8}$ where $\varepsilon$ denotes that this is a continuum $f$ channel, experiences a strong change with energy from threshold to $-30 \mathrm{eV}$ above threshold (see Chapter III). This behavior can be related to the changing overlap of the initial state with the continuum $\ell=3$ wavefunction; the overlap is poor at low kinetic energies because of the centrifugal repulsion of the $\ell=3$ wavefunction by the atomic nucleus, but increases at higher energies. This interaction of the photoelectron with the atomic potential causes the $\mathrm{Kr}$ 3d asymmetry paraneter to vary rapidly in this energy range. Similar, but more dramatic, effects can be observed when the potential experienced by the photoelectron contains a barrier as a result of the interplay of the Coulomb attraction and the centrifugal repulsion between the nucleus and the photoelectron. The barrier enhances the overlap of the initial and continuum states at certain energies, leading to very strong oscillations in both the cross section and asymmetry parameter. These changes are referred to as shape-resonance phenomena, because of their dependence on the shape of the potential. Measurements on the atomic-like I $4 \mathrm{~d}$ subshell in $\mathrm{CH}_{3} \mathrm{I}^{9}$ (Chapter IV) indicate that the cross section is low near threshold, wrere the photoelectron can be thought of as being trapped by the potential barrier, and increases ten-fold at higher energies. The I 4d asymmetry parameter also shows large changes in this energy range. 
Shape resonances also influence molecu?ar photoionization, with the important difference that the molecular potential is intrinsically anisotropic. Therefore, molecular shape-resonance behavior, particularly changes in the asymmetry parameter, may provide a fingerprint of the molecular potential. Another important difference between atomic and molecular shape resonances is that the vibrational modes available to molecules tend to weaken the effects of shape resonances on cross sections and asymmetry parameters. Chapter $v$ presents the first photoemission measurements of any gas-phase species near the $\mathrm{N}$ is threshold. The results for $\mathrm{N}_{2}$ and $\mathrm{NO}$ are interpreted in terms of shape-resonance phenomena.

Another category of single-electron effects can be understood by reference to Eq. (3). If a term in the summation changes sign by virtue of a change in wavefunction overlap with energy, that term will be zero at some energy, as initially elucidated by Cooper. 10 The result is a local minimum in the cross section at the energy of the zero crossing. If the term affected is the dominant one, a pronounced Cooper minimum in the cross section is observed and large changes in the as ymetry parameter will occur. This interpretation is used to describe the cross-section and asymmetry-parameter results for the I 4d subshell of $\mathrm{CH}_{3} \mathrm{I}$ (Chapter IV) for photon energies above $-150 \mathrm{eV}$. Photoionization processes that involve more than one electron generally occur with less irtensity than those involving only a single electron, making experimental measurements more difficult. Nevertheless, where feasible, these experiments yield a wealth of 
information on electron-electron correlation and intershell interactions. An example of the latter can be found in Chapter IV, where photoionization of the $\mathrm{I} 4 \mathrm{p}$ subshell in $\mathrm{CH}_{3} \mathrm{I}$ is discussed. The behavior of the I $4 p$ asymmetry parameter is remarkable because it closely mimics the I 4d asymmetry parameter, suggesting that an intimate coupling between the $4 d$ and $4 p$ subshells is present. Partial understanding of this phenomenon is possible with the realization that the I $4 p$ peak is not produced by a single-electron process, but can be identified to some extent with a multi-electron process yielding a $4 d^{8} 4 f$ final state. This measurement documents one of the most pronounced interchannel interactions observed to date.

Electron correlation leads to a breakdown of the koopmans picture of photoelectron spectra; peaks appear that correspond to the ionization of one electron accompanied by the excitation of a second electron to a higher-lying orbital. These 'satellite' peaks are usually less intense ( $\leq 20 \%)$ than the main single-electron peaks, and their intensities are derived solely from multi-electron interactions. Although much work has been done to determine the energies and configurations of satellite peaks, very little is known (experimentally or theoretically) about the energy dependences of their cross sections and angular distributions. In the nigh-energy or sudden limit, the shake-up mode ${ }^{11}$ has been used with some success in predicting satellite intensities. At lower energies, for which the photoelectron may interact significantly with the remaining electrons, processes other than shake-up may be important, and the exact identification of 
the contributions to satellite formation is beyond the present understanding. The results for satellites in He (Chapter VI) and $\mathrm{Kr}$ (Chapter III) constitute a considerable fraction of the existing energy-dependent satellite measurements. In both cases, changes in the cross sections and asymmetry parameters with photon energy have been observed. For He, these changes are explained in terms of an additionai low-energy mechanism for satellite production (other than shake-up) that is most important near threshold.

The photoionization studies of the He satellites also include measurements of the resonance behavior of cross sections and asymmetry parameters. The resonances are Rydberg-like states [eg. $\mathrm{He}^{\star}(3 \mathrm{~s} 3 \mathrm{p})$ ] in which both electrons are excited. The $\mathrm{He}^{*}$ atom then decays 0 the $\mathrm{He}^{+}$ion by the autoionization process; one electron escapes, the other electron relaxes to a $2 \mathrm{~s}$ or $2 \mathrm{p}$ orbital, leaving the $\mathrm{He}^{+}(\mathrm{n}=2)$ satelite states. In general, autoionization is an interference phenomenon because of the two pathways, direct ionization and ionization through the intermediate resonant state, by which the system can pass from the initial to the final state. The interference causes asymmetric profiles in both the cross section and asymetry parameter as a function of energy (see Chapter VI) that are quantitatively indicative of the matrix elements governing the autoionization process, and in favorable cases such as He, some of these matrix elements can be extracted from the experimental results.

Ti,e next chapter presents some experimental details that are common to the results discussed in Chapters III-VI. 


\section{REFERENCES}

1. T. Koopmans, Physica 1, 104 (1933).

2. D.W. Turner, A.D. Baker, C. Baker, and C.R. Brundle, Molecular Photoelectron Spectroscopy (Wi ley, New York, 1970).

3. K. Siegbahn, C. Nordling, G. Johansson, J. Hedman, P.F. Hedén, K. Hamrin, U. Gelius, T. Bergmark, L.O. Werme, R. Manne, and Y. Baer, ESCA Applied to Free Molecules (North-Holland, Amsterdam, 1969).

4. J. Berkowitz, Photoabsorption, Photoionization, and Photoelectron Spectroscopy (Academic Press, New York, 1979).

5. This is the dipole approximation (also referred to as neglect of retardation). It is a good assumption for photon energies used in this work $(\leq 600 \mathrm{eV})$. See Ref. 6 .

6. S.T. Manson, in Advances in Electronics and Electron Phys ics 41 , 73 (1976).

7. C.N. Yang, Phys. Rev. 74, 764 (1948).

8. The selection rules for atomic photoionization require that an $n 2$ bound electron be ionized to an $\varepsilon(\ell \pm 1)$ continuum state. The $\ell+1$ channel is usualiy dominant.

9. The effect of the methyl group on $I 4 d$ ionization is small, and the results in Chapter IV are explained adequately in terms of an atomic-like shape resonance.

10. J.W. Cooper, Phys. Rev. 128, 681 (1962).

11. Shake-up refers to the photoionization model in which the photoelectron has high kinetic energy and leaves the ionic core 
quickly. The remaining electrons are 'shaken' by the sudden change in potential, in some cases being excited to form satellite states. 


\section{I. EXPERIMENTAL}

Gas-phase photoelectron spectroscopy requires the coupling of a photon source, an apparatus to deliver the gaseous sample for interaction with the radiation, and an electron energy analyzer. For the experiments described in this dissertation, monochromatized synchrotron radiation was used as the photon source, gases were delivered by an effusive gas jet, and the electrons were detected by time-of-flight (TOF) analysis. All of the components and methods used for these experiments have been described previously, ${ }^{1-7}$ therefore this chapter will review only the major aspects of the technique. All of the measurements were made at the Stanford Synchrotroil Radiation Laboratory (SSRL) during discrete 'runs' approximately two weeks long a few times a year. This type of schedule was conducive to long hours, little sleep, and hectic experiments. Nonetheless, during my tenure, 11 runs were performed with the TOF apparatus (see Table 1, Chapter Il of Ref. 5), which included over 30 successful experiments. Figure 1 is a photograph of the individuals who were responsible for most of these successes.

The characteristics of synchrotron radiation have been described by Winick. ${ }^{8}$ Briefly, the radiation produced by SPEAR is a moderately intense continuum source from visible to hard $x$-ray wavelengths. Additional advantages are provided by the inherent polarization of sunchrotron radiation and the excellent time structure $10.3 \mathrm{nsec}$ pulses every 780 nsec in single-bunch running) available at SPEAR. 
Some disadvantages of working with synchrotron radiation pertinent to gas-phase photoelectron spectroscopy are discussed by Southworth. 3 I share with him 'the wonder of it all' when everything worked at the same time. Somehow it seemed to make working at SSRL worth it. After leaving the storage ring, the synchrotron radiation is deflected to a monochromator for energy dispersion and selection. All of the experiments in this dissertation were performed with the grazing-incidence Rowland-circle 'grasshopper' monochromator on Beam Line III-1 ${ }^{9}$ at SSRL. It was operated with a $1200 \mathrm{line} / \mathrm{mm}$ holographicaliy-ruled grating, yielding a useful energy range of 50 to maybe as high as $600 \mathrm{eV}$ (at the higher energies, the photon resolution is very poor). The monochromator is equipped with adjustable entrance and exit slits that allow the experimenter to select the desired photon resolution, while of course, giving up significant amounts of photon flux.

The monochromatized photon beam then enters the TOF chamber and intersects an effusive gas jat of the sample to be studied. The electrons emitted in the interaction region are detected by identical TOF analyzers at two angles, $0^{\circ}$ and $54.7^{\circ}$, with respect to the polarization vector of the radiation. The ovarall scheme is depicted in Fig. 2. The gas jet normally was positioned vertically with the nozzle below the photon beam. Typical pressures in the interaction region were estimated to be $1-5 \times 10^{-3}$ torr, approximately an order of magnitude larger than the ambient chamber pressure. The pressure behind the jet's nozzle was monitored by a capacitance manometer (to 
better than $\pm 5 \%$ ) that also automatically controlled the flow rate of the gas into the chamber. To protect the ultrahigh-vacuum monochromator and storage ring from the $-10^{-4}$ torr in the experimental chamber, it was necessary to interpose an ultrathin Al window $(1500 \AA$ thick) to separate the two vacuum regions. To protect this window, an elaborate inter lock system (pressure sensors, fast-closing valves, etc.) was developed. We pride ourselves in never having broken one during regular operation, although at other (less dangerous) times, the delicate windows seemed particularly susceptible to clumsiness. The separation of our vacuum system from that of SSRL permitted moderately worry-free running, but with the disadvantage of some photon absorption. The transmission of the Al window is shown in Fig. 3. The structure at $72 \mathrm{eV}$ is due to the $L_{2,3}$ edge of $A 1$ metal. This scan was taken by monitoring the photon flux at the back of the TOF chamber with a sodium salicylate scintillator and an optical photomultiplier tube ( $R C A$ 8850). The response of the sodium salicylate is constant up to $120 \mathrm{ev},{ }^{10}$ above which no measurements are available. Initial indications are that the response increases at higher photon energies, which would require corrections to be made to some of our cross-section measurements.

Electrons emitted in the interaction region were energy analyzed by virtue of the different flight times, $t$, for electrons with different kinetic energies, $\varepsilon$, according to

$$
t=\frac{C L_{t}}{e^{1 / 2}}
$$


where $L_{t}$ is the length of the flight path of the TOF analyzers $(\sim 23$ $\mathrm{cm})$, and $\mathrm{C}$ is a constant. This method works at SSRL because the synchrotron radiation is pulsed as a result of the bunched structure of the electrons in the storage ring. The pulses are narrow $\{\sim 0.3$ nsec) and spaced far enough apart in time (195-780 nsec depending on the running mode of SPEAR) to allow electrons of most energies to be collected between consecutive pulses with moderate-to-good time resolution. The limitation on the TOF analyzer resolution at low electron kinetic energies is the uncertainty in the flight path (and hence the flight time) because of the finite width $(3 \mathrm{~mm})$ of the photon beam at the interaction region. For the grasshopper monochromator, the resolution was typically $2.5 \%$ of the kirietic energy of the electrons for the $0^{\circ}$ analyzer, and 3.5-4.0\% for the $54.7^{\circ}$ detector. At high kinetic energies ( $>100-200 \mathrm{eV})$, other contributions to the resolution become important, and the poor overall analyzer resolution is prohibitive to doing well-resolved experiments.

The TOF method is well-suited to gas-phase photoelectron spectroscopy because it collects nearly all energies of photoelectrons simultaneously, providing an excellent signal-to-noise ratio for many experiments. Simultaneous measurement of photoelectron peak intensities at two angles yields asymmetry parameters (see Eq. 4, Chapter I) that are independent of variations in the photon flux and gas pressure. Peak intensities at $54.7^{\circ}$ (the 'magic angle'), for which $P_{2}(\cos \theta)$ vanishes, are directly proportional to photoionization cross sections after normalization to photon flux and gas 
pressure. Comparison of intensities of two peaks in the same $54.7^{\circ}$ spectrum (branching ratios) alleviates the problem of normalization. In order to place the asymmetry-parameter measurements on the correct scale, it is necessary to calibrate the apparatus by measuring known values of 3 . For this purpose, we used the asymmetry parameters for Ne $2 p$ and $2 s$ ionization, 11 deriving from them a curve descriting the relative efficiency of the two analyzers operating in the double-angle TOF mode. This procedure has been described in detail previously. ${ }^{3-7}$ It is important to note that this greatly reduces the effects of systematic errors on the measurements of unknown asymmetry parameters. For example, the effect of some nonlinear polarization on the $B$ measurements is virtually negligible as long as the degree of linear polarization is greater than $-75 \%$. The actual polarization of the grasshopper monochromator has been estimated to be $98 \% 3,7$ Total systematic errors for a for all of the measurements in this dissertation are estimated to be \pm 0.10 or less. A similar correction must be applied to the cross-section and branching-ratio measurements because of the energy-dependent transmission of the $54.7^{\circ}$ analyzer. The transmission is determined simultaneously with the efficiency function by using the known values of the cross section for Ne L-shell ionization. 11 systematic errors for the cross-section and branchingratio results are estimated to be $10-15 \%$ of the reported value for each data point.

The remaining chapters deal with individuai experiments that were performed during the Fall of 1981 and the Spring of 1982; $\mathrm{kr}$ (Chapter 
III), $\mathrm{CH}_{3}$ I (Chaptér IV), $\mathrm{N}_{2}$ and NO (Chapter V), and He (Chapter VI). Additional experimental details pertinent to a given experiment may be found in these chapters. 


\section{REFERENCES}

1. M.G. White, Ph.D. Thesis, University of California, Berkeley (1979); Lawrence Berkeley Laboratory report No. LBL-9527.

2. M.G. White, R.A. Rosenberg, G. Gabor, E.D. Poliakoff, G. Thornt on, S.H. Southworth, and D.A. Shirley, Rev. Sci. Instrum. 50, 1268 (1979).

3. S.H. Southworth, Ph.D. Thesis, University of California, Berkeley (1982); Lawrence Berkeley Laboratory report No. LB!-13512.

4. S. Southworth, C.M. Truesdale, P.H. Kobrin, D.H. Lindle, W.D. Brewer, and D.A. Shirley, J. Chem. Phys. 76, 143 (1982).

5. P.H. Kobrin, Ph.D. Thesis, University of California, Berkaley (1983); Lawrence Berkeley Laboratory report No. LBL-15591.

6. C.M. Truesdale, Ph.D. Thesis, University of California, Berkeley (1983); Lawrence Berkeley Laboratory report No. LBL-15902.

7. S.H. Southworth, U. Becker, C.M. Truesdale, P.H. Kobrin, D.W. Lindle, S. Owaki, and D.A. Shirley, Phys. Rev. A 28, 261 (1983).

8. H. Winick, in Synchrotron Radiation Research, ed. by $\mathrm{H}$. Winick ano S. Doniach (Plenum, New York, 1980).

9. F.C. Brown, R.Z. Bachrach, and N. Lien, Nucl. Instrum. Meth. 152, 73 (1978).

10. J.A.R. Samson and G.N. Haddad, J. Opt. Soc. Am. 64, 1346 (1s74). 11. F. Wuilleumier and M.O. Krause, J. Electron Spectrosc. 15, 15 (1979). 


\section{FIGURE CAPTIONS}

Fig. 1. Picture taken during the Spring of 1981 at SSRL. Frori left: Carlton "Trues" Truesdale, Dennis Lindle, Uwe Becker, Steve Southworth, and Paul Kobrin.

Fig. 2. Schematic of the double-angle time-of-flight (DATOF) method.

Fig. 3. The relative photon flux from the grasshopper monochrom tor after passing through the $A 1$ window (solid curve). The dashed curve is the relative flux through a vitreous-carbon window that has been used only since the present experiments were sompleted. Both scans were taken as described in the text. The dip in flux at $\sim 280 \mathrm{eV}$ for the $\mathrm{Al}$ window is due to the K-edge absorption of carbon adsorbed on the surfaces of the optical elements in the beam line. 


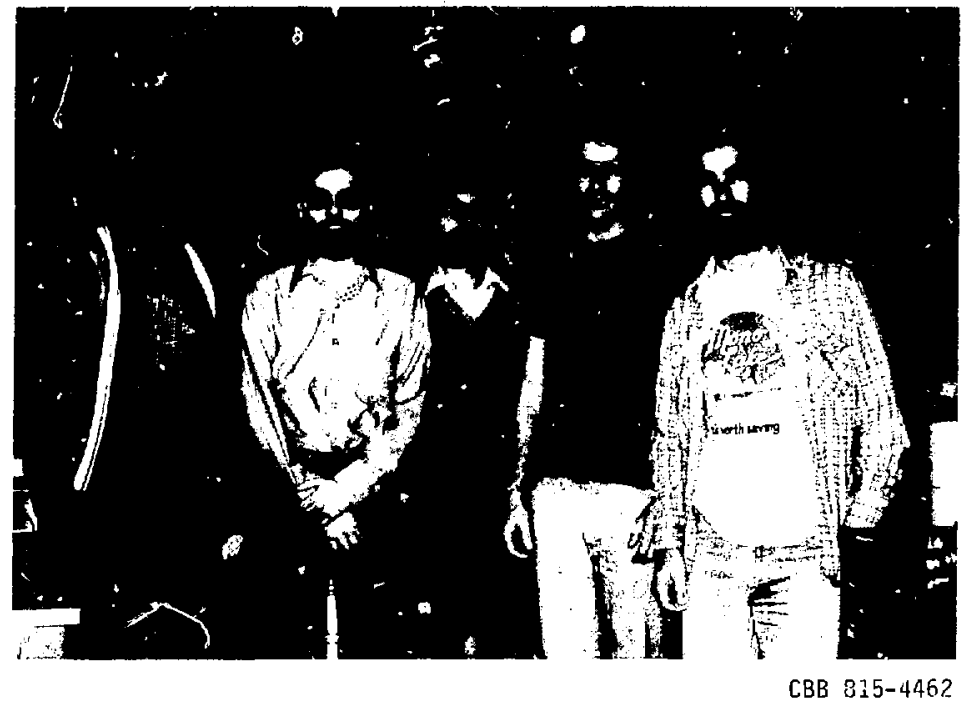

Figure 1 


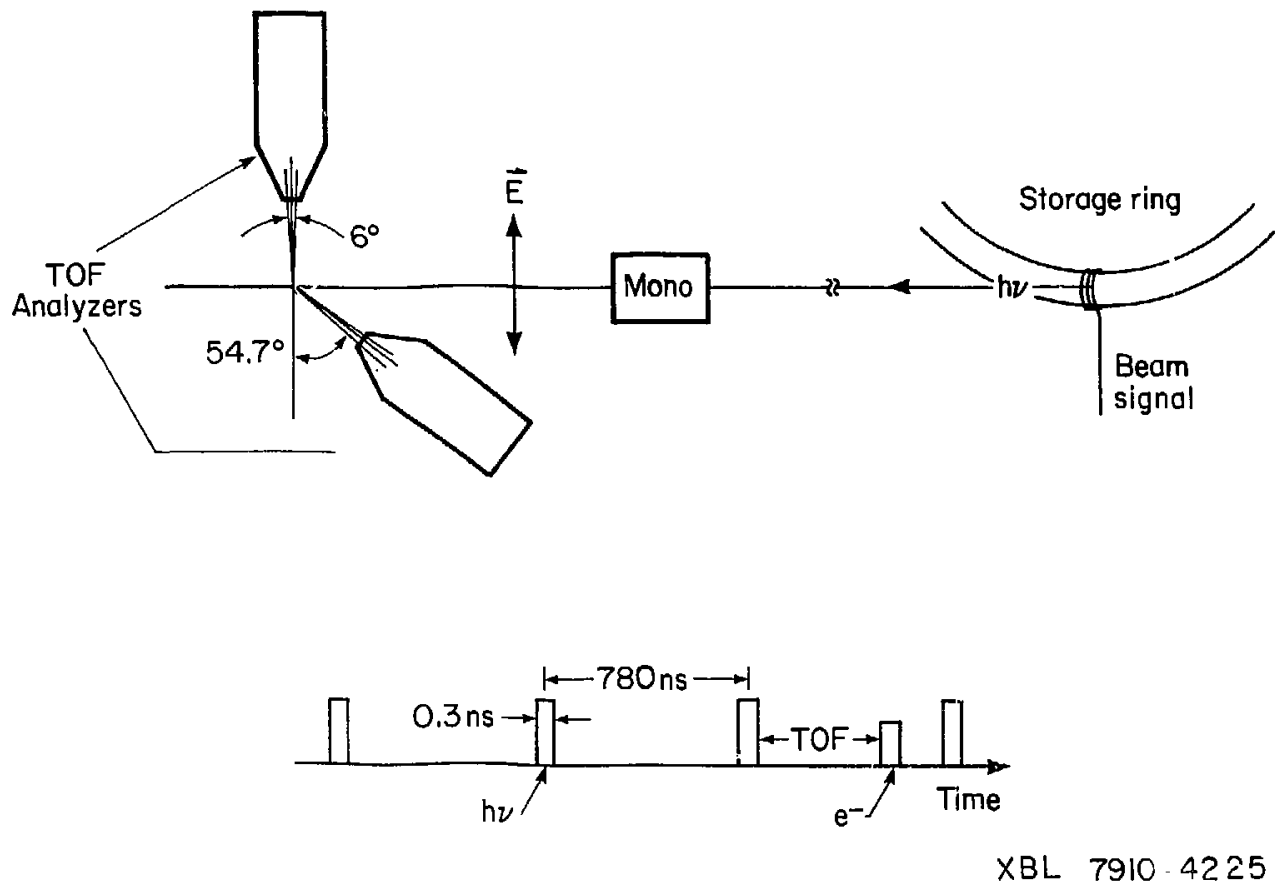

Figure 2 
$-21-$

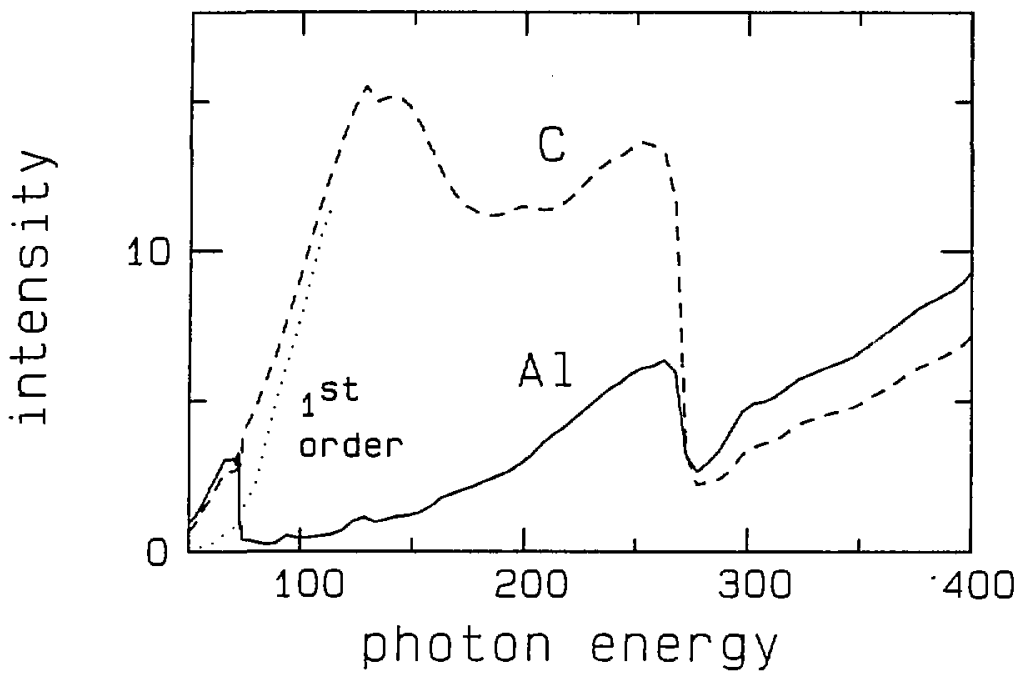

Figure 3 
III. PHOTOEMISSION FROM THE 3d SUBSHELL OF ATOMIC Kr*

Recent photoemission measurements on the inner shells of $\mathrm{Xe},{ }^{1,2}$ $\mathrm{Hg},{ }^{3}$ and $\mathrm{I}$ in $\mathrm{CH}_{3} \mathrm{I}^{4}$ have exhibited oscillations in the energydependences of angular distributions that are caused by interaction of the photoelectron with the atomic potential. In particular, a centrifugal barrier produces a strong shape-resonance effect in the asymmetry parameters for the $4 d$ and $4 f$ states in the above systems. Although no centrifugal barrier is present for atomic $\mathrm{Kr}$ because of the smaller Coulomb attraction in this lower- $z$ element, similar effects are expected based on the shape of the repulsive part of the potential. 5 We report here measurements confirming this expectation for the $\mathrm{Kr}$ 3d asymmetry parameter. Also included are results indicating the importance of multi-electron effects; interchannel coupling of the $3 d$ and $3 p$ subshells, and production of satellites of the $3 d$ main line. For the latter, relative intensities and asymmetry parameters are presented which show significant changes as a function of energy.

A TOF spectrum of $\mathrm{Kr}$ taken at $224 \mathrm{eV}$ photon energy is shown in Fig. 1. This spectrum is dominated by features associated with $3 d$ subshell photoionization; the unresolved $3 d$ photoemission 1 ines with binding energies of $93.7 \mathrm{eV}\left(4 \mathrm{~d}_{5 / 2}\right)$ and $94.9 \mathrm{eV}\left(4 \mathrm{~d}_{3 / 2}\right),{ }^{6}$ a satellite peak of the $3 d$ line, mostly composed of $4 p \rightarrow 5 p$ shake-up states ( $-114 \mathrm{ev}$ binding energy ${ }^{7}$ ), and all of the Auger features below 60 ev kinetic energy. Evidence of $3 p$ ionization (thresholds at 
214.4 and $222.2 \mathrm{ev}^{6}$ ) is apparent with the $\mathrm{M}_{2,3} \mathrm{M}_{4,5} \mathrm{~N}$ Auger peak. The remaining high-energy peaks result from photoionization of the valence subshells and from photoemission induced by higher-order components of the synchrotron radiation.

Figure 2 displays the measured asymmetry parameters for 3d photoionization along with previous measurements by $\mathrm{Krause}^{8}$ and Carlson et al. ${ }^{9}$ At certain photon-energy settings of the monochromator, a component of second-order radiation (energy of $2 \mathrm{hv}$ ) was large enough to produce peaks in our spectra, primarily second-order peaks of $\mathrm{Kr}$ 3d photoionization. Because the $\mathrm{B}$ measurements are independent of the photon flux, we were able to extend our $\beta_{3 d}$ results to higher photon energies. At low energies, we observe excellent agreement with the earlier data, ${ }^{9}$ whereas the earlier higher-energy results ${ }^{8}$ are systematically larger than the present data. Comparison with HartreeFock velocity $(H F-V)^{10}$ and relativistic random-phase approximation $\left(\right.$ RRPA) ${ }^{11}$ calculations also is made in Fig. 2. The theoretical curves nearly coincide from threshold to $125 \mathrm{ev}$, both showing good agreement with the experimental results. Above $200 \mathrm{eV}$, the HF-V calculation overestimates the asymmetry parameter measured here, but agrees rather well with the earlier measurements. 8 The minimum in $B_{3 d}$ at $115-120 \mathrm{eV}$ can be ascribed to a potential-barrier effect on the ef photoelectron channel. 5,12 Near threshold, the centrifugal barrier for an $2=3$ continuum wavefunction inhibits the ef channel. As the energy increases, this channel rapidly begins to dominate the photoionization process, including a subsequent change in the 
asymmetry parameter. ${ }^{13}$ The concurrence of the theoretical results with the experimental measurements at low energy confirms this interpretation, as well as indicating that $\mathrm{Kr} 3 \mathrm{~d}$ photoionization is adequately described by a one-electron model.

The single-electron picture seems to break down near $210 \mathrm{eV}$. The 3d asymmetry parameter experiences a resonance effect apparently associated with the onset of $3 p$ ionization. Because this feature appears below the $3 p_{3 / 2}$ ionization threshold at $214.4 \mathrm{eV}$, we assign this interchannel interaction as autoionization of a Rydberg level(s) involving excitation of a $3 p$ electron. Three such levels have been observed in absorption, ${ }^{14}$ the resonances at $210.7 \mathrm{eV}(3 \mathrm{p}+5 \mathrm{~s})$ and $213.2 \mathrm{eV}(3 \mathrm{p}+6 \mathrm{~s}, 4 \mathrm{~d})$ being the likely candidates for the effect observed here.

The intensity of the $3 d 4$ pnp satellite peaks (underline refers to the hole states) relative to the $3 d$ main line is shown in the top of fig. 3. The $4 p+n p$ satellites were unresoived in the Tof spectra: thus the results in Fig. 3 represent values for all of these peaks combined. Very little is known about the energy-dependent behavior of satellite intensities. ${ }^{15}$ Empirically, Wuilleumier and Krause ${ }^{16}$ have plotted satellite relative intensities against a reduced-energy parameter, $\varepsilon / E_{0}$, where $\varepsilon$ is the kinetic energy of the satellite photoelectron, and $E_{0}$ is the satellite excitation energy (i.e. the binding energy of the satellite less the binding energy of the main line). For the $\mathrm{kr} 3 d 4$ pnp satellites, $E_{0}$ is approximately $20 \mathrm{eV}$, and the reduced-energy region covered by the measurements in Fig. 3 is 
$3.5 \leq \varepsilon / E_{0} \leq 8.2$. At the upper end of this range, one suspects the high-energy or sudden limit to be reached. ${ }^{16}$ our high-energy value of $7.8(6) \%$ agrees very well with an Al Ka measurement ${ }^{17}$ of $8(1) \%$ for the sum of the $4 p \rightarrow n p$ satellites. However, higher-resolution measurements with $\mathrm{Mg} \mathrm{Ka} \mathrm{radiation}{ }^{7}$ yielded $11.7 \%$ for these same transitions, which is in better agreement with the theoretical sudden-1imit result ${ }^{7}$ of $10.6 \%$. Because the earlier measurements did not take the asymmetry parameter into account, they must be interpreted with caution. At lower energies we find the relative intensity to be larger than our high-energy value, illustrating that other processes besides shake-up may become important for lower values of the reduced energy. We note that the relative satellite intensity is decreasing in an energy range in which the $3 d$ cross section is decreasing also [as determined from absorption measurements ${ }^{18}$ and the fact that $3 d$ subshell absorption dominates in this energy range (see Fig. 1)].

Even less is known about the energy dependence of satellite asymmetry parameters. As a first approximation, one might expect that the satellite $\beta$ will mimic the asymmetry parameter of the main line. Comparison of the $3 d 4$ pnp asymmetry parameter results in Fig. 3 to B $_{3 d}$ (Fig. 2) shows that both a parameters increase in this energy range, but the slope for the satellite asymetry parameter is about a factor of 2 larger than for ${ }_{3} d^{*}$. This intriguing result cannot be explained in the context of shake-up calculations.

For photon energies between 225 and $285 \mathrm{eV}$, evidence of $3 p$ photoionization appeared in our spectra in the form of an $\mathrm{M}_{2,3} \mathrm{M}_{4,5} \mathrm{~N}$ 
Auger feature. These Auger transitions have been found to contribute approximately $50 \%$ to the decay of $3 p$ vacancy states. ${ }^{19}$ Consequently, we can estimate that $3 p$ subshell absorption accounts for about $10 \%$ of the total absorption cross section in this energy range. The $3 p$ photoemission peak itself was discernible for energies from 275 to 285 $\mathrm{eV}$. The $3 \mathrm{p}$ asymmetry parameter was determined to be $0.26(8)$ in this range, lower than the HF-V result ${ }^{10}$ of 0.5 at these energies. The measured value of $a_{3 p}$ is nearly the same as $\beta_{3 d}$ at the same photon energies. A similar result has been observed for the $I 4 p$ and $4 d$ subshells in $\mathrm{CH}_{3} \mathrm{I} .{ }^{4}$ in that work, recourse was made to strong many-electron interactions in the $I 4 p$ subshell in an attempt to understand this phenomenon. Ohno and Wend $\mathrm{n}^{20}$ have discussed the same types of effects for the $\mathrm{Kr} 3 \mathrm{p}$ subshell. The asymmetry parameter for the $M_{2,3} M_{4,5} \mathrm{~N}$ Auger feature is $0.0(1)$. Likewise, the asymmetry parameters for the $M_{4,5}$ NN Auger peaks were found to be approximately zero over the energy range studied. 


\section{REFERENCES}

*Work done in collaboration with P.H. Kobrin, C.M. Truesdale, P.A. Heimann, T.A. Ferrett, U. Becker, H.G. Kerkhoff, and D.A. Shirley 1. S.H. Southworth, P.H. Kobrin, C.M. Truesdale, D. Lindle, S. Owaki, and D.A. Shirley, Phys. Rev. A 24, 2257 (1981).

2. S. Southworth, U. Becker, C.M. Truesdale, P.H. Kobrin, D.H. Lindle, S. Owaki, and D.A. Shirley. Phys. Rev. A 28, 261 (1983).

3. P.H. Kobrin, P.A. Heimann, H.G. Kershoff, D.W. Lindle, C.M. Truesdale, T.A. Forrett, U. Becker, and D.A. Shirley, Phys. Rev. A 27, 3031 (1983).

4. D.W. Lindle, P.H. Kobrin, C.M. Truesdale, T.A. Ferrett, P.A. Heimann, H.G. Kerkhoff, U. Becker, and D.A. Shirley (Chapter IV). 5. S.T. Manson and J.W. Cooper, Phys. Rev. 165, 126 (1968). 6. K. Siegbahn, C. Nordling, G. Johansson, J. Hedman, P.F. Hedén, K. Hamrin, U. Gelius, T. Bergmark, L.O. Werme, R. Manne, and Y. Baer, ESCA Applied to Free Molecules (North-Holland, Amsterdam, 1969).

7. D.J. Bristow, J.S. Tse, and G.M. Bancroft, Phys. Rev. A 25,1 (1982).

8. M.O. Krause, Phys. Rev. 177, 151 (1969).

9. T.A. Carlson, M.O. Krause, F.A. Grimm, P.R. Keller, and J.W. Taylor, Chem. Phys. Lett. $\underline{\text { 87, }} 552$ (1982).

10. D.J. Kennedy and S.T. Manson, Phys. Rev. A 5,227 (1972).

11. K.-N. Huang, W.R. Johnson, and K.T. Cheng, At. Data Nucl. Data Tables $\underline{26}, 33$ (1981).

12. J.W. Cooper and S.T. Manson, Phys, Rev. 177, 157 (1969). 
13. This effect is not a shape resonance, but simply the energy-dependent behavior of $3 d$ photoionization into the $\ell=3$ continuum channel. This behavior is governed strongly by the centrifugal potential experienced by an ef continuum wavefunction at low kinetic energy. Related measurements that do involve shape resonances (potential barriers) can be found in Refs. 1-4.

14. W.S. Watson and F.J. Morgan, J. Phys. B 2, 277 (1969). 15. P.H. Kobrin, S. Southworth, C.M. Truesdale, D.W. Lindle, U. Becker, and D.A. Shirley, Phys. Rev. A 29 (1984); D.W. Lindle, T.A. Ferrett, U. Becker, P.H. Kobrin, C.M. Truesdale, H.G. Kerkhoff, and D.A. Shirley (Chapter VI), and references therein. 16. F. Wuilleumier and M.0. Krause, Phys. Rev. A 10, 242 (1974). 17. D.P. Spears, H.J. Fischbeck, and T.A. Carlson, Phys. Rev. A $\underline{9}$, 1603 (1974).

18. G.V. Marr and J.B. West, At. Data Nucl. Data Tables $\underline{18}, 497$ (1976); J.P. Connerade and M.W.D. Mansfield, Proc. R. Soc. Lond. A343, 415 (1975); A352, 557 (1977); J.B. West and G.V. Marr, ibid A349, 397 (1976).

19. M.0. Krause and T.A. Carlson, Phys. Rev. 149, 52 (1966).

20. M. Ohno and G. Wendin, J. Phys. B 11, 1557 (1978). 


\section{FIGURE CAPTIONS}

Fig. 1. TOF photoelectron spectrum of $\mathrm{Kr}$ at a photon energy of $224 \mathrm{eV}$ and with $\theta=0^{\circ}$. All of the features below $60 \mathrm{eV}$ are $M_{4,5^{N N}}$ Auger lines. The $3 d$ satellite includes all of the $4 p \rightarrow n p$ shake-up transitions. The peaks to the right of the $3 \mathrm{~d}$ main line arise from valence photoionization and from photoemission induced by second- and higher-order components of the incident radiation.

Fig. 2. Angular-distribution asymmetry parameter for $\mathrm{kr} 3 \mathrm{~d}$ photoemission. Solid circles are the present results, open circles and X's are from Refs. 9 and 8, respectively. The solid and dashed curves are RRPA (Ref. 11) and HF-V (Ref. 10) calculations, respectively. The RRPA curve has been shifted 9 eV to lower energy. The two calculations nearly coincide for photon energies from threshold to $125 \mathrm{eV}$, at which point the RRPA curve stops.

Fig. 3. Intensity relative to the $3 \mathrm{~d}$ main 1 ine (top) and asymmetry parameter (bottom) for the $\mathrm{kr} 3 \mathrm{~d} 4 \mathrm{p}$ np satellites. All of the $4 p \rightarrow n p$ satellites were unresolved and are included in these results. 


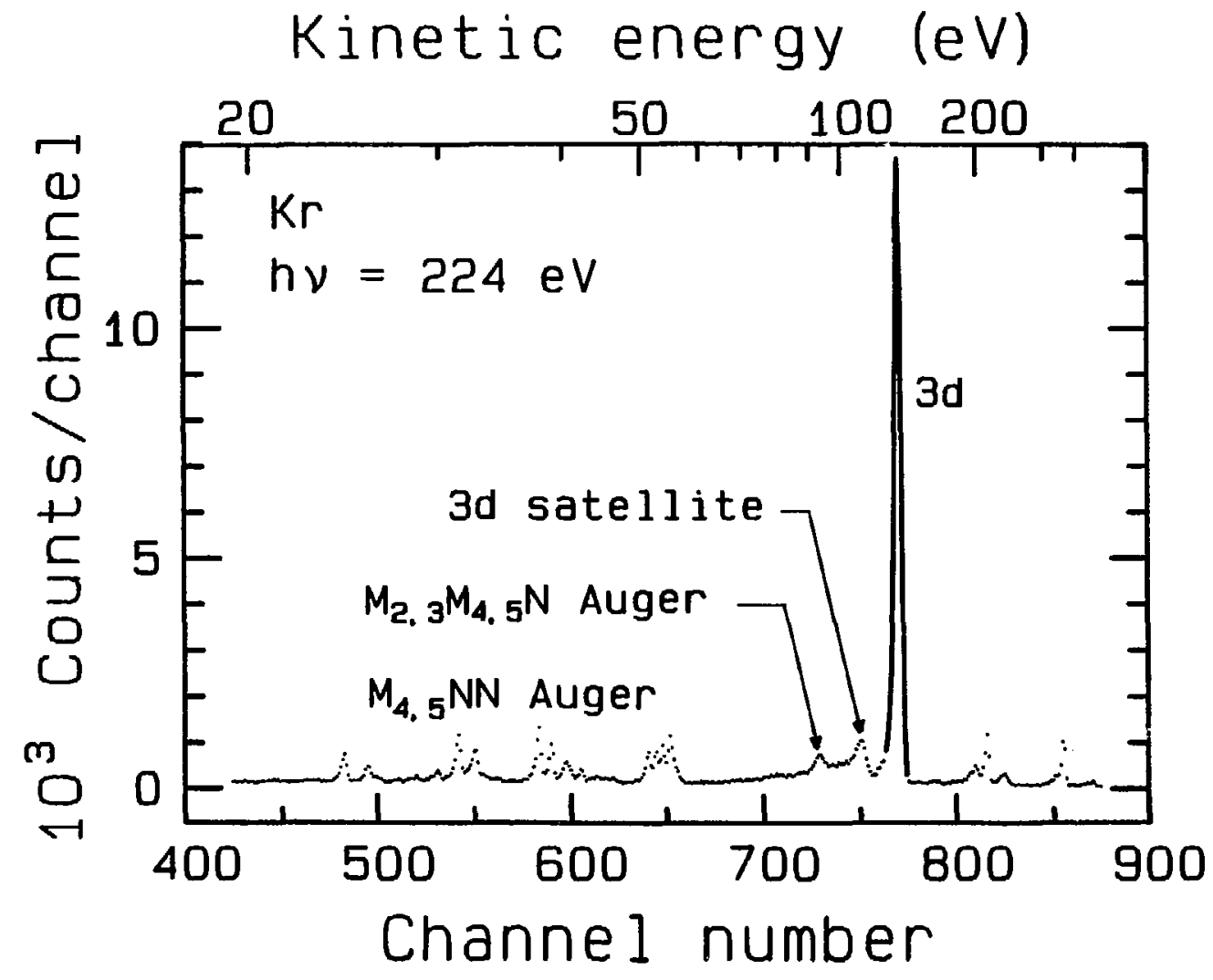




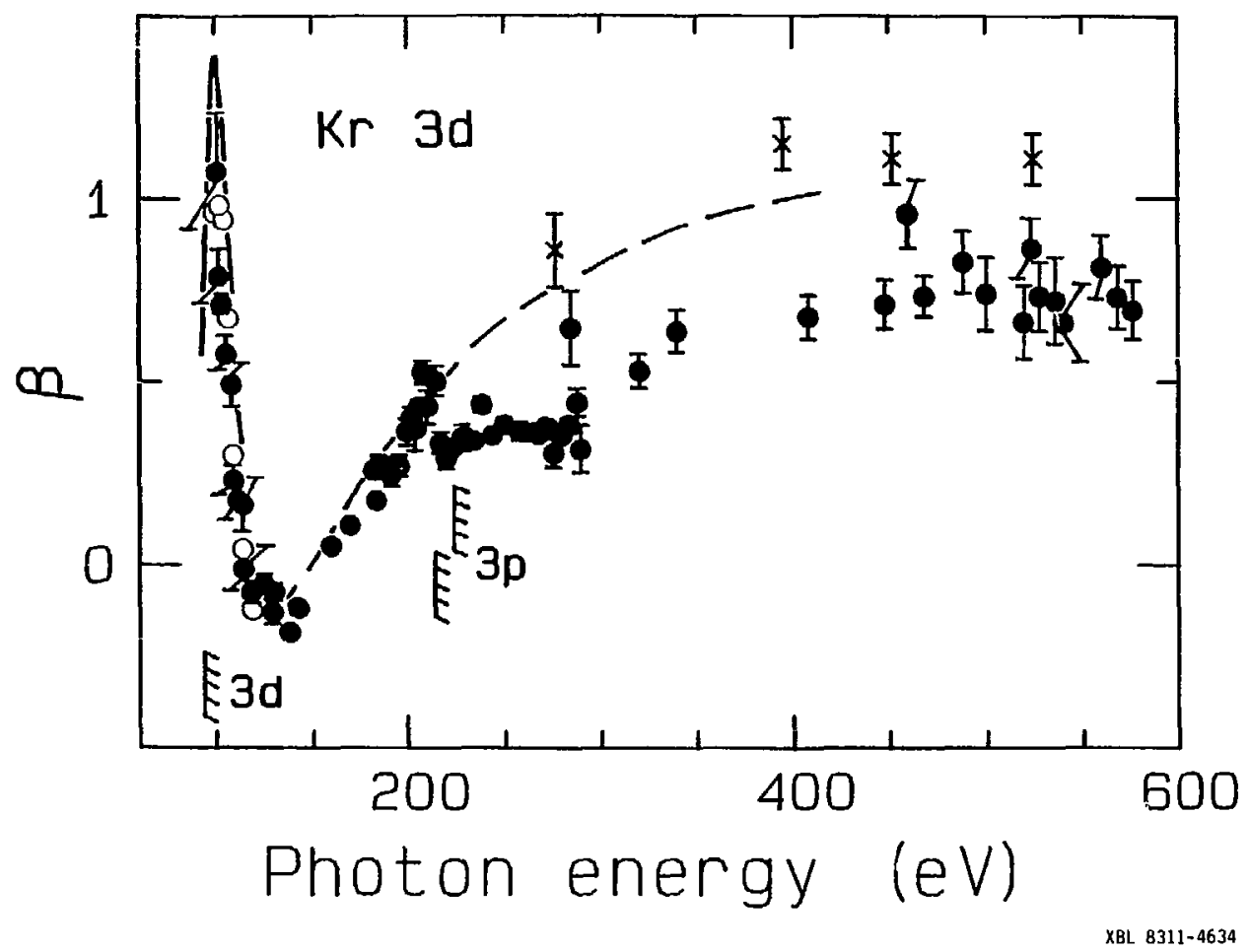

Figure 2 


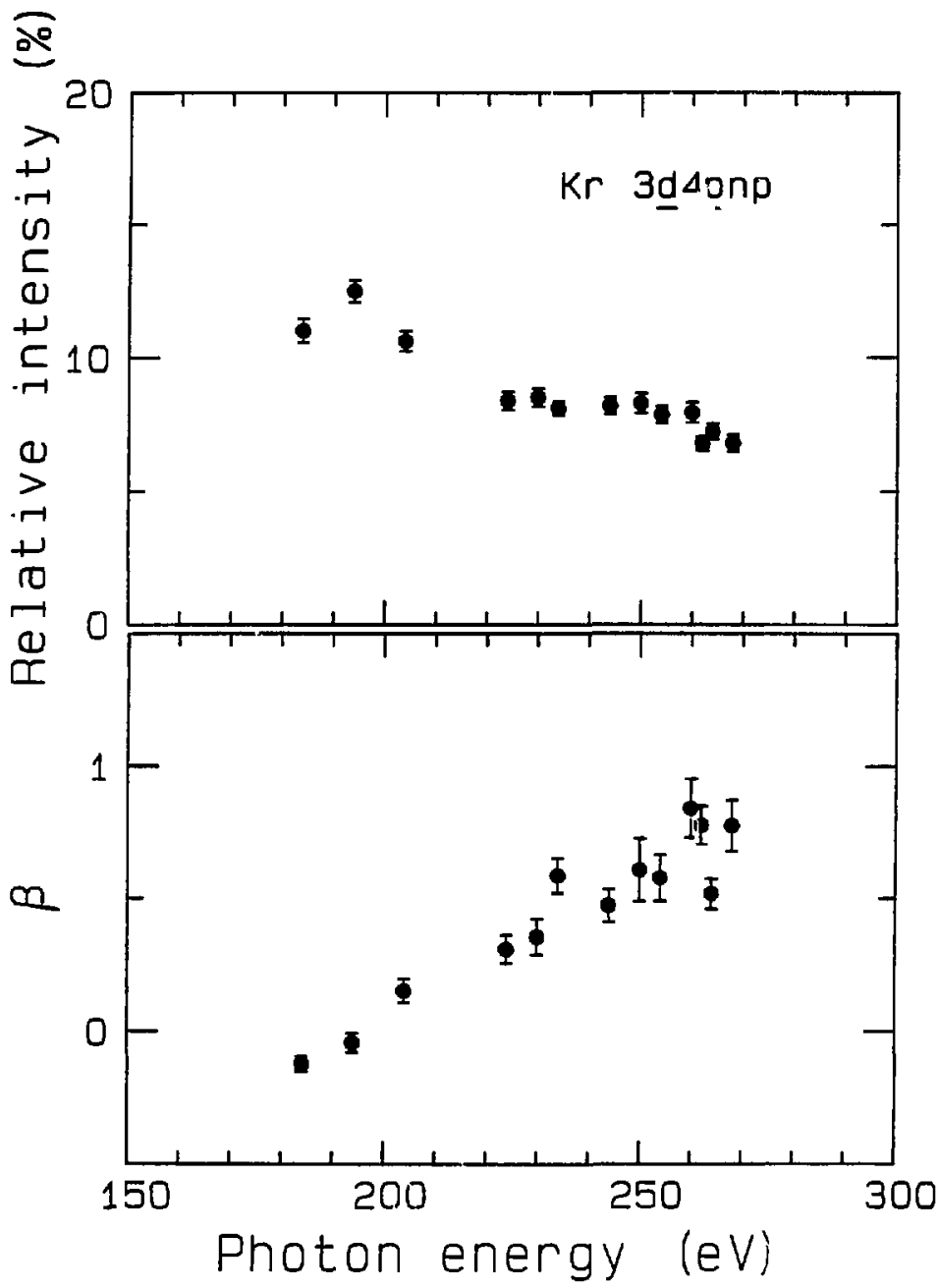

XBL 8311-4636

Figure 3 
IV. INNER-SHELL PHOTOEMISSION FROM THE IODINE ATOM IN $\mathrm{CH}_{3} I^{*}$

\section{A. INTRODUCTION}

Recent photoelectron-spectroscopy (PES) studies of $x e^{1-8}$ have highlighted both one-electron and multi-electron effects that are important in vescribing photoionization in the $\mathrm{N}$ and 0 shells of $\mathrm{Xe}$ above the $4 \mathrm{~d}$ threshold. Elucidation of these effects has been accomplished by comparison of the experimental results to progressively more detailed theoretical calculations..$^{9-20}$ One result has been to identify $X e$ and other elements with similar $Z$ as especially good systems for exhibiting some of these effects. The present photonenergy-dependent PES study of the iodine atom in $\mathrm{CH}_{3} \mathrm{I}$ adds considerably to the available experimental measurements for elements near $X e$ in the periodic table.

For photon energies immediately above the $4 d$ ionization threshold $\left(\mathrm{Xe}^{7,8}\right.$ or 1$)$, the photoelectron spectrum is dominated by features associated with 4d-vacancy states. It is known ${ }^{7}$ that photoemission from the $x e$ ad subshell can be described accurately by considering a series of single-electron effects that appear in the $4 d+$ ef continuum channel; a Coulomb phase shift occurs near threshold, followed at somewhat higher enersy by a shape resonance resulting from the trapping of the outgoing photoelectron by a centrifugal barrier, and finally at still higher energy, the $4 d+$ ef dipole matrix element experiences a change in sign and causes a Cooper minimum. Experimen- 
tally, pronounced changes corresponding to these phenomena have been seen in the subshell cross section, ${ }^{2}$ the spin-orbit branching ratio, ${ }^{4,5}$ and the angular distribution of xe $4 \mathrm{~d}$ photoelectrons. 3,7 To the extent that the iooine atom in a molecule such as $\mathrm{CH}_{3} \mathrm{I}$ exhibits atomic-like behavior, the present measurements yield information about the $Z$ dependence of the one-electron effects peculiar to $4 d \rightarrow_{\varepsilon f}$ transitions. The present results indicate that the inner subshells of $\mathrm{I}$ in $\mathrm{CH}_{3} \mathrm{I}$ are atomic-like. The I $4 \mathrm{~d}$ subshell shows behavior remarkably similar to Xe $4 d$, with some small systematic variations. These results are discussed in Sect. Bl.

Multi-electron effects generally become more important for the less intense (smaller cross section) features in photoelectron spectra. This is especially true for the ' $4 p$ ' shells of the elements in the series Te to $\mathrm{Ba}{ }^{1,16}$ quotation marks are used here because the final states that have binding energies in the vicinity of the $4 p$ thresholds can be described adequately r:nly in terms of collective effects; ${ }^{16}$ hence designation as a $4 p$ final state is inappropriate. In xe for example, only one major discrete peak is observed in this energy range aiong with continuum-like structure, ${ }^{1}$ contrary to the one-electron model prediction of two peaks corresponding to the $4 \mathrm{p}_{3 / 2}$ and $4 \mathrm{p}_{1 / 2}$ ionic states. The prominent peak has been attributed primarily to the $4 d^{8} 4 f$ configuration, and the remaining structure is explained in terms of the near-degeneracy of the $4 p-v a c a n c y$ states with $4 d^{8} n$ s singly-ionized and $4 d^{8}$ doubly-ionized states. ${ }^{16}$ As this near-degeneracy is enhanced (or removed) by observing different $Z$ 
elements, significant changes in the ' $4 \mathrm{p}$ ' spectra are seen.' 1 For I, the discrete structure is less intense than in $\mathrm{Xe}$, and the identification of the single observed peak as a $4 p$-hole state is even more questionable. The cross section and angular distribution of the I ' $4 p$ ' peak as functions of photon energy are presented here for the first time (note that similar measurements have not yet been made for $\mathrm{Xe}$ ' $4 \mathrm{p}^{\prime}$ ). The asymmetry-parameter results are nearly identical to the I $4 \mathrm{~d}$ results at the same photon energies. Though interpretation of these results is not straightforward, the assignment of the final state as $4 d^{8} 4 f$ is consistent with the results discussed in Sect. 82 .

\section{B. RESULTS AND DISCUSSION}

A representative TOF photoelectron spectrum of $\mathrm{CH}_{3} \mathrm{I}$ at a photon energy of $64 \mathrm{eV}$ is shown in Fig. 1. The predominance in this spectrum of features associated with I 4d ionization is apparent. The Auger peaks $\left(\mathrm{N}_{4,5} \mathrm{~W}\right)$ result from the decay of the $4 d$-yacancy states. Both Auger features receive contributions from many decay channels, and no attempt is made here to assign any of these Auger transitions. The following subsections treat in turn each subshell from which we were able to detect photoelectrons; I $4 d$ in Sect. 81 and I ' $4 p$ ' in Sect. 82 .

1. I 4d SUBSHELL

Cross-section and asymmetry-parameter results for the I $4 \mathrm{~d}$ 
subshell are shown in Figs. 2 and 3 . The $14 d$ cross section has been scaled to the absolute photoabsorption measurement of 0'Sullivan ${ }^{21}$ at the maximum in the cross section. The peak value in the absorption cross section is approximately $17 \mathrm{Mb}$. At certain photon-energy settings of the monochromator, a component of second-order radiation (i.e. an energy of $2 h_{v}$ ) was large enough to produce additional peaks in our spectra, primarily second-order peaks of I $4 \mathrm{~d}$ photoelectrons. Because the $s$ measurements are independent of the photon flux, we were able to use these spectra to extend our ${ }_{4} 4$ results to higher photon energies. The points obtained in this way are shown as open circles in Fig. 3.

The data in Figs. 2 and 3 are for the unresolved I 4 d peak. Measurements in which the spin-orbit components, $4 d_{5 / 2}$ and $4 d_{3 / 2}$, are resolved at low kinetic energies are presented in Fig. 4 . The binding energies of the $4 d_{5 / 2}$ and $4 d_{3 / 2}$ states are 56.7 and 58.3 ev, respectively. 21

Interpretation of the results for the I $4 d$ subshell benefits from the previous measurements ${ }^{2-5,7,8}$ and calculations $9-13,17,19,20$ for the $\mathrm{Xe}$ 4d subshell. The I $4 d$ cross section shows two important effects. At $85-90 \mathrm{eV}$, the cross section reaches a maximum which can be attributed to a centrifugal-barrier shape-resonance effect in the $4 d \rightarrow$ cf outgoing channel. 22 The position of the maximum agrees with the absorption measurement of O'Sullivan. The second effect occurs at 1.65-170 eV photon energy and can be assigned to a Cooper minimum ${ }^{23}$ in the same $4 d \rightarrow \varepsilon f$ channel. Both of these phenomena in the I $4 d$ 
subshell occur at the same photoelectron energy $(\neq 5 \mathrm{eV})$ as in the $\mathrm{Xe}$ 4d case. ${ }^{24-29}$ This concordance for the Cooper minima is based on comparison of the asymmetry-parameter data ${ }^{7}$ because no measurements of the partial cross section at the Xe 4d Cooper minimum are available. An atomic shape resonance is caused by a centrifugal barrier in the potential experienced by the ionized (or excited) electron. For energies below the peak of the barrier, the effect $c$ an be considered as a trapping of the electron. Typically, these barriers occur only for $\ell \rightarrow \ell^{\prime}=\ell+1$ channels, in this case $4 d+\varepsilon f$, because the strength of the centrifugal repulsion scales as $\ell^{\prime}\left(\ell^{\prime}+1\right)$, where $2^{\prime}$ is the orbital angular momentum of the electron in the final state. The nature of the oarrier is sensitive to the interplay of the attractive coulomb potential and the repulsive centrifugal potential. In a singleelectron central-field mode 1,22 these potentials depend upon the values of $Z$ and $\ell, 1$, respectively. Certain combinations of these parameters will produce centrifugal barriers; others will not. When a barrier is present, the qualitative behavior of the cross section as a function of energy can be discerned from the following argument. $^{22}$ Considering only one-electron processes, the magnitude of the dipole matrix element that determines the single-channel cross section is proportional to the overlap between the initial bound one-electron wavefunction and the final continuum wavefunction. Near threshold, the centrifugal barrier excludes the continuum wavefunction from the interior of the atom and very little overlap occurs. As the available energy approaches the height of the barrier, the amplitude 
for penetration of the continuum wavefunction into the interior of the atom increases quickiy, causing a rapid increase of its overlap with the bound wavefunction. Accompanying this penetration is a phase shift of the continuum wave. These features constitute the shape resonance. At still higher energies the barrier plays a less important role, and the dipole matrix element gradually decreases with energy.

For $d+$ ef transitions, the shape resonance is most pronounced for the elements in the periodic table centered around $\mathrm{xe},{ }^{22}$ because the $4 f$ orbital has a high degree of continuum character in this sequence of atoms, so that virtual excitation to this orbital contributes significantly to the photoionization process. ${ }^{30}$ This is apparently a general phenomenon. For the particular case of I $4 d+\varepsilon f$, we are interested in the implications of the $Z$ dependence of 4d cross sections. Compared to Xe, the centrifugal barrier in I is somewhat higher ( $Z$ is lower). This should cause the shape resonance to peak at a higher kinetic energy than in Xe. From a wavefunction overlap argument presented in Ref. 31 , a second result of the higher barrier in I is to reduce the cross-section maximum compared to Xe. Absorption measurements for the atomic species in the series

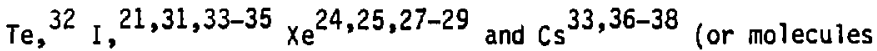
containing these atoms) imply a decrease of the kinetic energy of about 5 eV per series member for the shape-resonance maximum (approximately 40, 35, 30 and $25 \mathrm{eV}$, respectively). A photoemission measurement ${ }^{2}$ of the $x e 4 d$ cross section was consistent with the absorption result, but could not confirm it because of the scarcity of 
data points. Likewise, the present measurement and another using electron energy-loss spectroscopy ${ }^{39}$ are consistent with the photoabsorption peak measured for $\mathrm{CH}_{3} \mathrm{I}^{21}$ (32 eV above threshold) and $\mathrm{I}_{2}^{31}$ ( $34 \mathrm{eV}$ above threshold). However, our result also is consistent with the kinetic energy of the maximum in the $x_{e}$ absorption 24,25,27 (30 eV) to within experimental error. We note the $2 \mathrm{eV}$ difference between the peaks of the $\mathrm{CH}_{3} \mathrm{I}$ and $\mathrm{I}_{2}$ absorption results and suggest that it is due to molecular effects.

A definite minimum is observed in the I $4 d$ cross section which falls to a value of $\sim 0.1$ Mb at $165-170$ eV photon energy, then increases by a factor of 2 at higher energy. This minimum, which we interpret as a Cooper minimum arising from a sign change in the $4 d+$ ef matrix element, occurs at the same kinetic energy ( $\pm 5 \mathrm{eV})$ as the Cooper minimum in the $\mathrm{Xe} 4 \mathrm{~d}$ subshell, as determined by comparison of the asymetry-parameter results. ${ }^{7}$ No other observations of Cooper minima in $4 d$ subshe 11 cross sections are available for further comparison.

The I $4 d$ asymmetry-parameter results shown in Fig. 3 also are affected strongly by the shape-resonance and Cooper-minimum phenomena. The trapping of the outgoing photoelectron by the centrifugal barrier and the consequent phase shift are interpreted as causing the initial decrease in the measured $4 \mathrm{~d}$ asymmetry. Because this happens before the barrier is ov arcome, the minimum in $\beta_{4 d}$ occurs at $15-20$ eV lower kinetic energy than the maximum in the $4 \mathrm{~d}$ cross section, or at approximately 70 eV photon energy. 
Using the Cooper-zare formula ${ }^{40}$ for $B$ in LS coupling, $B_{4 d}$ is predicted to be 0.1 at the Cooper minimum if spin-orbit and multielectron effects are neglected. ${ }^{41}$ This value for $\beta_{4 d}$ is reached at a photon energy between 165 and $170 \mathrm{eV}$, in complete agreement with the minimum in the cross-section results.

The theoretical curves $12,19,20$ in Fig. 3 represent calculations for the $x e$ 4d subshell that have been shifted $11 \mathrm{eV}$ to lower energy for comparison with the I $4 d$ results at the same kinetic energy. A detailed comparison of these curves to experiment for $X e$ has been given in Ref. 7. Overall, the similarity between the $I 4 d$ and $X e 4 d$ results (experimental and theoretical) is remarkable, especially in the region of the Cooper minimum which is predicted best by the relativistic random-phase approximation (RRPA) 20 and Dirac-Fock $(D F)^{19}$ calculations. At lower energies, around 70 and $150 \mathrm{eV}$, differences between the $I 4 d$ results and the $x \in 4 d$ predictions are more adparent. This observation can be understood in the light of the di $-r$ c...es between the shape resonances in $X e$ and I described above. umpared to the experimental $x e 4 d$ results, we note a general tendency for I $\beta_{4 d}$ to be less than $x_{e} \beta_{4 d}$ by $0.2-0.3$ at all energies, a result for which we have no explanation. Near threshold, no experimental evidence for the predicted initial increase in I $B_{4 d}$ is seen, in agreement with the $x e 4 d$ results.

One additional observation concerning the $\beta_{4 d}$ results pertains to the tiwo points at 120 and $125 \mathrm{eV}$. Both of these points deviate significantly (by 0.3 or more) from a smooth curve connecting the 
remainder of the data. Because of the proximity of the I ' $4 p$ ' threshold at approximately $129 \mathrm{eV}$, we tentatively explain this behavior as autoionization of Rydberg states leading to this threshold. Clearly more experimental work with a finer mesh of points would be needed to confirm this conjecture.

At the lowest photon energies used in this work, the spin-orbit components of the I $4 \mathrm{~d}$ peak were resolved (see Fig. 1). The $4 \mathrm{~d}_{5 / 2^{-}}$ $\mathrm{t}_{0}-4 \mathrm{~d}_{3 / 2}$ intensity ratio is shown in $\mathrm{Fig}$. 4. It starts at 6 at 60.5 $\mathrm{eV}$ and quickly drops to the statistical value of 1.5. This behavior is satisfactorily explained by a kinetic-energy effect; at a given photon energy, the $4 d_{5 / 2}$ peak has $1.6 \mathrm{eV}$ higher kinetic energy than the $4 d_{3 / 2}$ peak, and thus is further along in its sharp increase in cross section caused by the shape resonance (see Fig. 2). The asymmetry parameters of the individual components are shown in the bottom of Fig. 4. They follow the sane trend as the unresolved $B_{4 d}$ in Fig. 3. The differences between $B_{5 / 2}$ and $B_{3 / 2}$ also are explained by the kinetic-energy effect.

An additional peak in the photoelectron spectra with a binding energy of $72(1) \mathrm{eV}$ appeared at photon energies from 110 to $130 \mathrm{eV}$. This peak can be attributed to a correlation satellite(s) of the $I 4 d$ main line. It probably corresponds to final states with a $4 d$ vacancy and a valence electron promoted from a nonbonding orbital localized on the I atom $\left(E_{3 / 2}\right.$ or $\left.E_{1 / 2}\right)$ to a higher-lying antibonding valence orbital. The intensity of the satellite relative to the $\mathrm{I} 4 \mathrm{~d}$ line is $15(4)^{\alpha}$, in this energy range. It appears strongly at these photon 
energies probably because it experiences the same shape-resonance effect as the I $4 \mathrm{~d}$ cross section.

\section{I ' $4 p$ ' SUBSHELL}

Svensson et al. ${ }^{1}$ recorded Al Ka photoelectron spectra of the series of elements $T e$ to $B a$ in the regions of their respective $4 p$ binding energies. Rather than finding two peaks $\left(4 p_{3 / 2}\right.$ and $\left.4 p_{1 / 2}\right)$ corresponding to single-electron transitions to the final state, each of these elements showed distinct multi-electron behavior to var'ing degrees; Te showing the largest effect, Ba the least. Wendin and Ohno ${ }^{16}$ explained the situation for $x e$ in terms of strong manyelectron effects that prevent the existence of an isolated $4 p_{3 / 2}$ - or $4 p_{1 / 2}$-hole state, but require that a ' $4 p$ ' vacancy actually appears primarily as the $x e^{+} 4 d^{8} 4 f$ state. This strong coupling results from the near-degeneracy of a single $4 p$ hole and a double vacancy with two $4 d$ holes. A similar description is based on the onset of energyallowed $\mathrm{N}_{2,3} \mathrm{~N}_{4,5} \mathrm{~N}_{4,5}$ super-Coster-Kronig decay in the range of $Z$ from $52(\mathrm{Te})$ to $56(\mathrm{Ba}) .16$

Because the TOF detectors record photoelectrons over a wide range of energies simultaneously, we obtained several spectra of the I '4p' region of $\mathrm{CH}_{3}$ I while making the measurements on the I $4 \mathrm{~d}$ subshell. The '4p' spectra looked very similar to that of Svensson et al., and our measured binding energy of $129(1)$ el agrees with theirs. The ' $4 p$ ' cross-section and asymetry-parameter results for photon energies from 
175 to $300 \mathrm{eV}$ are shown in Fig. 5. These values were determined by considering only the area under the single prominent $\left(4 d^{8} 4 f\right)$ peak and by excluding as much as possible the broad continuum-like structure at higher binding energy (see Fig. 5 of Ref. 1 for a spectrum of $\mathrm{CH}_{3} \mathrm{I}$ in this region). He interpret the data in $\mathrm{Fig} .5$ as primarily representing the $4 d^{8} 4 f$ final state.

The I ' $4 p$ ' cross-section data in the top of Fig. 5 were scaled to the previously scaled I $4 d$ cross section. The intensity of the $4 d^{8} 4 f$ peak accounts for only $25-50 \%$ of the total intensity in the ' $4 p$ ' binding-energy region. The remainder is contained in the broad continuum. Taking this extra intensity into account, the total I '4p' cross section is approximately equal to the I 4 d cross section in this photon-energy range.

The I ' $4 p$ ' asymmetry-parameter results (Fig. 5) start at -0.3 at $175 \mathrm{ev}$, drop to a minimum of -0.6 at $190 \mathrm{eV}$ and reach 0.5 by $300 \mathrm{eV}$. Included in Fig. 5 is one curve representing a Hartree-Fock velocity (HF-V) calculation ${ }^{12}$ of $X e B_{4 p}$, shifted in energy to coincide with the I ' $4 p$ ' threshold, and a second derived from a smooth curve through the I $\beta_{4 d}$ data in Fig. 3. The HF-V curve, which predicts the Dehavior of $\beta_{4 p}$ in a single-electron approximation, does not agree with the data, whereas the latter curve fits the I $s_{4 p}$ results very well, suggesting the possibility of strong interchannel coupling between the $4 d$ photoemission channels and the channels that lead to the ' $4 p$ ' peak. Interchannel coupling is plausible if the $4 d^{8} 4 f$ state reached via the ' $4 p$ ' transition is regardeo as a multi-electron 
satellite of the $4 d^{9}$ final state. Identification of the exact mechanism by which these channels can couple through the continuum and lead to a $\beta_{4 p}$ similar to $\beta_{4 d}$ would require a more detailed theoretical model than any presently available.

\section{CONCLUSIONS}

Photon-energy-dependent measurements of the behavior of the $14 \mathrm{~d}$ and ' $4 \mathrm{p}$ ' subshells in $\mathrm{CH}_{3} \mathrm{I}$ have illustrated some important singleelectron and multi-electron effects. The I $4 d$ cross section and asymmetry parameter show pronounced changes under the influence of a shape resonance and a Cooper minimum in the $4 d \rightarrow$ ef outgoing photaelectron channel. Analogous results have been observed for the $\mathrm{Xe} 4 \mathrm{~d}$ subshe 11,7 indicating not only the atomic-like nature of the 1 atom in $\mathrm{CH}_{3} \mathrm{I}$, but yielding useful information about the $Z$ dependence of these one-electron effects on $4 d$-subshell ionization.

The first measurements as a function of photon energy for a ' $4 p$ ' subshell in the interesting elemental series Te-Ba have shown that interchannel coupling with the $4 d$ channel strongly perturbs the ' $4 p$ ' asymmetry parameter. Still to be understood is the nature and relative composition of the final states other than $4 d^{8} 4 f$ contributing to the 1 ' $4 \mathrm{p}$ ' peak, and how these states couple to the $14 \mathrm{~d}$ manifold to cause ${ }_{4 p}$ to be similar to $\beta_{4 d^{*}}$. Further experimental studies on ' $4 p$ ' subshells in this region of the periodic table, especially $Z=54$ (Xe), are needed to aid in this understanding. 


\section{REFERENCES}

*Wark done in collaboration with P.H. Kobrin, C.M. Truesdale, T.A. Ferrett, P.A. Heimann, H.G. Kerkhoff, U. Becker, and D.A. Shirley

1. S. Svensson, N. Mărtennson, E. Basilier, P.A. Malmquist, U. Gelius, and K. Siegbahn, Phys. Scripta 14, 141 (1976).

2. J.B. West, P.R. Woodruff, K. Codling, and R.G. Haulgate, J. Phys. B $\underline{9}, 407$ (1976).

3. L. Torop, J. Morton, and J.B. West, J. Phys. B 9,2035 (1976).

4. S.P. Shannan, K. Codling, and J.B. West, J. Phys. B 10 , 825 (1977).

5. M.S. Banna, M.0. Krause, and F. Wuilleumier, J. Phys. B 12, L125 (1979).

6. M.0. Krause, T.A. Carlson, and P.R. Woodruff, Phys. Rev. A $\underline{24}$, 1374 (1981).

7. S.H. Southworth, P.H. Kobrin, C.M. Truesdale, D. Lindle, S. Owaki, and D.A. Shirley, Phys. Rev. A 24, 2257 (1981).

8. S. Southworth, U. Becker, C.M. Truesdale, P.H. Kobrin, O.W. Lindle, S. Owaki, and D.A. Shirley, Phys. Rev. A 28, 261 (1983).

9. J.W. Cooper, Phys. Rev. Lett. 13, 762 (1964).

10. S.T. Manson and D.J. Kennedy, Chem. Phys. Lett. $\underline{7}, 387$ (1970).

11. S.T. Manson, Phys. Rev. Lett. 26, 219 (1971).

12. D.J. Kennedy and S.T. Manson, Phys. Rev. A $\underline{5}, 227$ (1972).

13. G. Wendin, J. Phys. B $\underline{6}, 42$ (1973).

14. M.Y. Amusia, N.B. Berezina, and L.V. Chernysheva, Phys. Lett. 51A, 101 (1975).

15. M.Y. Amusia and V.K. Ivanov, Phys. Lett. 59A, 194 (1976). 
16. G. Wendin and M. Ohno, Phys. Scripta 14, 148 (1976).

17. W.R. Johnson and V. Radojević, J. Phys. B 11, L773 (1978).

18. M.Y. Amusia, Comments At. Mol. Phys. 8,61 (1979).

19. W. Ong and S.T. Manson, Phys. Rev. A 21, 842 (1980).

20. K.-N. Huang, W.R. Johnson, and K.T. Cheng, At. Data Nucl. Data Tables 26, 33 (1981).

21. G. O'Sullivan, J. Phys. B 15 , L327 (1982).

22. S.T. Manson and J.W. Cooper, Phys. Rev. 165, 126 (1968).

23. J.W. Cooper, Phys. Rev. 128, 681 (1962).

24. R. Haensel, G. Keitel, P. Schreiber, and C. Kunz, Phys. Rev. 188, 1375 (1969).

25. _..L. Ederer and M. Manalis, J. Opt. Soc. Am. 도, 634 (1975).

26. A.P. Lukirskii, I.A. Brytov, and T.M. Zimkina, Opt. Spektrosk. 17, 438 (1964); [Opt. Spectrosc. $\underline{17}, 234$ (1964)].

27. D.L. Ederer, Phys. Rev. Lett. 13, 760 (1964).

28. T.M. Zimkina and S.A. Gribovskii, J. Phys. Coll. C4-10, 282 (1971).

29. J.P. Connerade, Proc. R. Soc. London, A347, 581 (1976).

30. For $Z=56(\mathrm{Ba})$, the $4 f$ orbital is bound, but unoccupied, and very strong intershell-correlation effects related to the shaperesonance effects for lighter elerients (eg. Xe, I) are important. See, for example; G. Wendin, J. Phys. E $\underline{9}$, L297 (1976); J.P. Connerade, Contemp. Phys. 19, 415 (1978) and references therein. 31. F.J. Comes, U. Nielsen, and W.H.E. Schwarz, J. Chem. Phys. $\underline{58}$, 2230 (1973).

32. T. Tuomi, B. Sonntag, and G. Zimmerer, DESY Report SR-72/14 (1972). 
33. F.C. Brown, C. Gähwiller, H. Fujita, A.B. Kunz, W. Scheifley, and N. Carrera, Phys. Rev. B 2,2126 (1970).

34. P.K. Carroll, E.T. Kennedy, and G. O'Sullivan, Appl. Opt. 19, 1454 (1980).

35. M. Pettini, M. Mazzoni, and G.P. Tozzi, Phys. Lett. $12 A, 168$ (1981).

36. M. Cardona, R. Haense 1, D.W. Lynch, and B. Sonntag, Phys. Rev. B 2, 1117 (i970).

37. H. Petersen, K. Radler, B. Sonntag, and R. Haensel, J. Phys. B $\underline{8}$, 31 (1975).

38. K. Radler and B. Sonntag, Chem. Phys. Lett. 39, 371 (1976).

39. A.P. Hitchcock =nd C.E. Brior, J. Electron Spectrosc. 13,193 (1978).

40. J. Cooper and R.N. Zare, J. Chem. Phys. 48, 942 (1968).

41. P.H. Kobrin, P.A. Heimann, H.G. Kerkhoff, D.W. Lindle, C.M.

Truesdale, T.A. Ferrett, U. Becker, and D.A. Shirley, Phys. Rev. A 27, 3031 (1983). 


\section{FIGURE CAPTIONS}

Fig. 1. TOF spectrum of $\mathrm{CH}_{3} \mathrm{I}$ at $64 \mathrm{eV}$ photon energy taken with the $54.7^{\circ}$ analyzer.

Fig. 2. Partial cross section of the $\mathrm{I} 4 \mathrm{~d}$ subshell in $\mathrm{CH}_{3} \mathrm{I}$, scaled at $85 \mathrm{eV}$ to the absorption measurement of O'Sullivan, Ref. 21. These data represent the sum of the cross sections for the spin-orbit components, $4 d_{5 / 2}$ and $4 d_{3 / 2}$, with an average binding energy of $57.5 \mathrm{eV}$. The photon resolution for the measurements in this work is a constant $1.3 \AA$ full-width at half maximum.

Fig. 3. Angular-distribution asymetry parameter of the I 4d subshell in $\mathrm{CH}_{3} \mathrm{I}$. The data are weighted averages of the asymmetry parameters for the $4 d_{5 / 2}$ and $4 d_{3 / 2}$ final states. Solid and open circles represent data taken with first-order and second-order light, respectively. Theoreticai calculations for the xe $4 d$ subshell shifted to coincide with the I $4 d$ threshold; solid curves are Hartree-Fock length (HF-L) and velocity (HF-V) by Kennedy and Manson, Ref. 12; short-dashed curve is Dirac-Fock (DF) by Ong and Manson, Ref. 19; and long-dashed curve is relativistic random-phase approximation (RRPA) by Huang et al., Ref. 20.

Fig. 4. Branching ratio (top) and asymetry parameters (bottom) for the spin-orbit components, $4 \mathrm{~d}_{5 / 2}$ and $4 \mathrm{~d}_{3 / 2^{\circ}}$. The point at $101.6 \mathrm{eV}$ in the bottom panel represents $B$ for both final states. 
Fig. 5. Partial cross section (top) and asymetry parameter (bottom) for I ' $4 \mathrm{p}$ ' photoelectrons from $\mathrm{CH}_{3} \mathrm{I}[129(1)$ eV binding energy]. The cross-section point at $250 \mathrm{eV}$ has been excluded because an Auger peak overlapped the ' $4 \mathrm{p}$ ' peak in the TOF spectrum at this photon energy. The curves in the bottom panel represent HF- $V$ calculations of Kennedy and Manson (solid), Ref. 12, and a smooth curve (dashed) drawn through the $\beta_{4 d}$ results in Fig. 3 . 


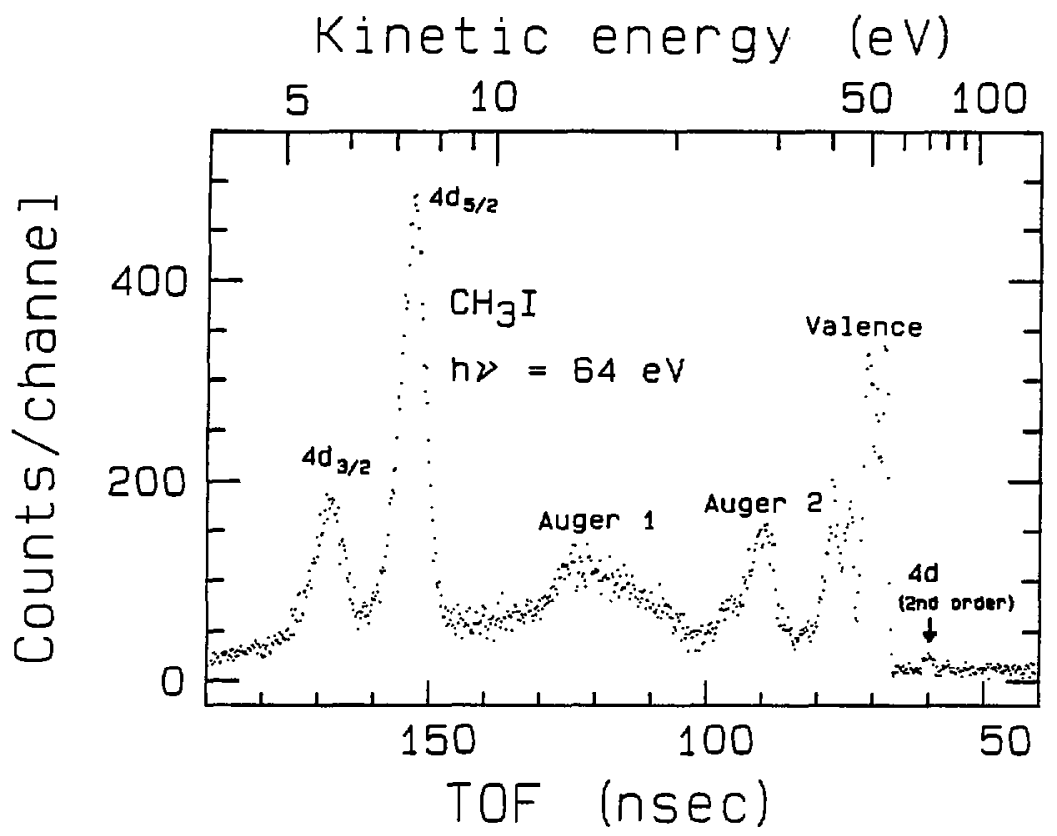

XEL 836-10196

Figure 1 


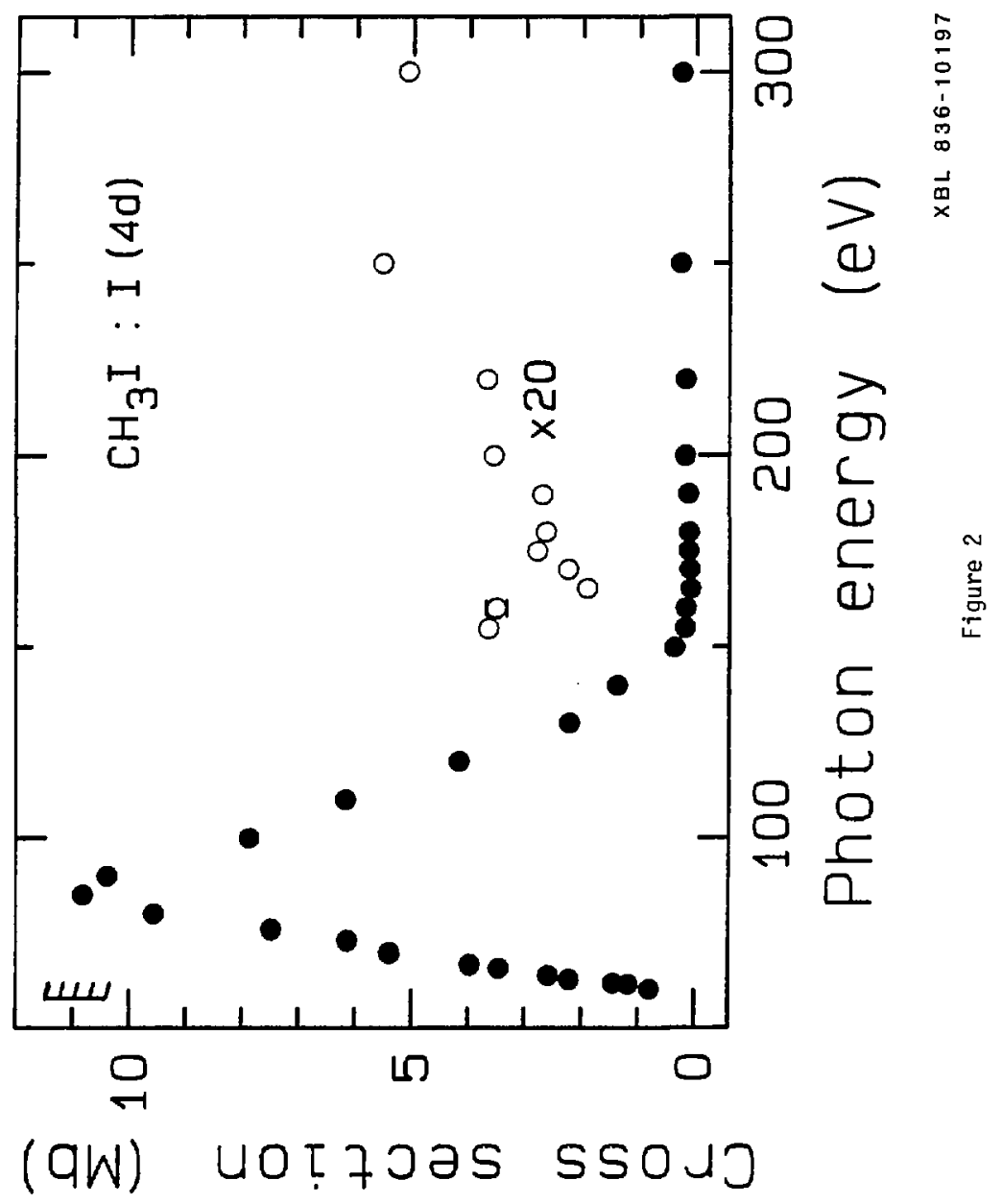




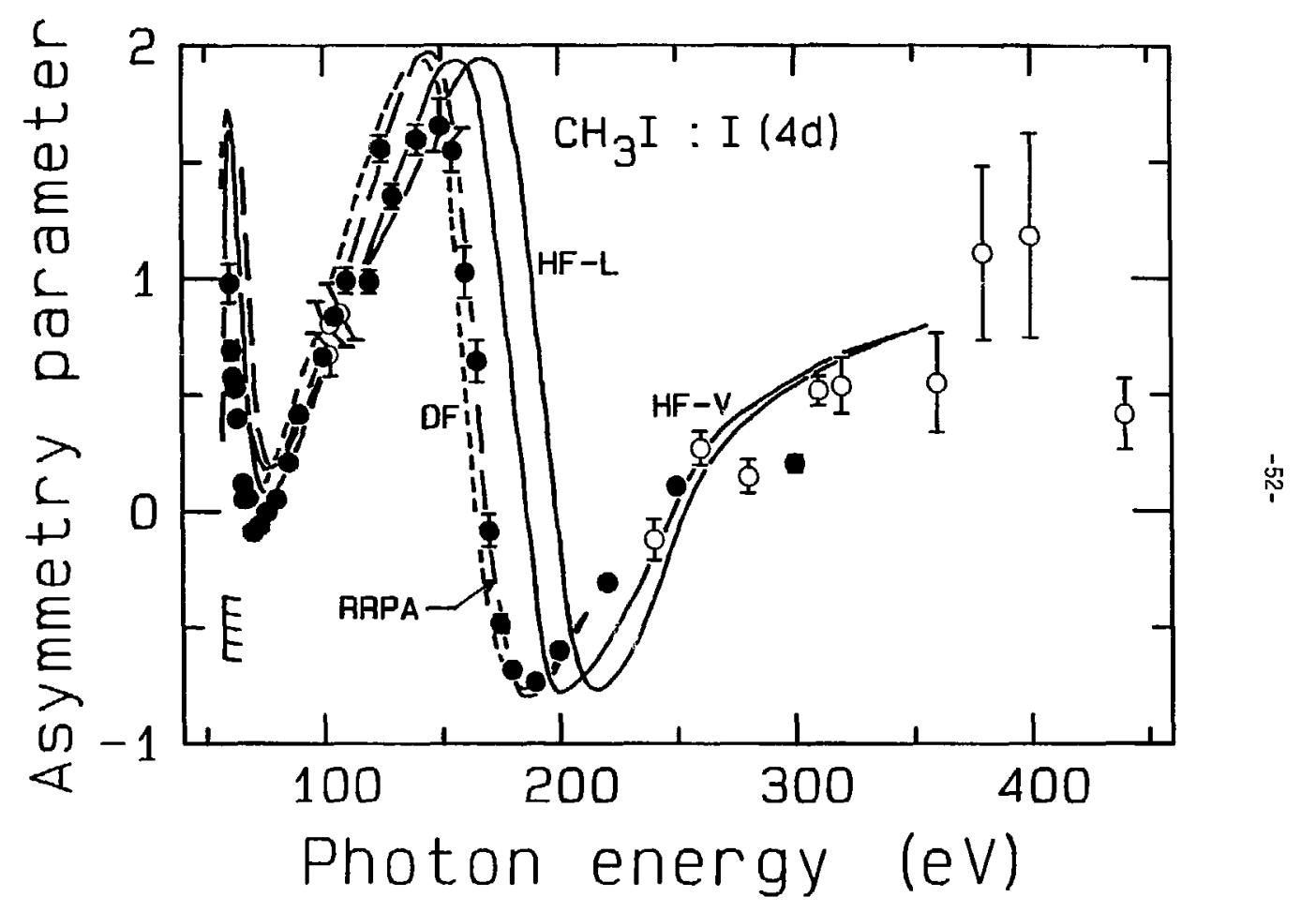

Figure 3 

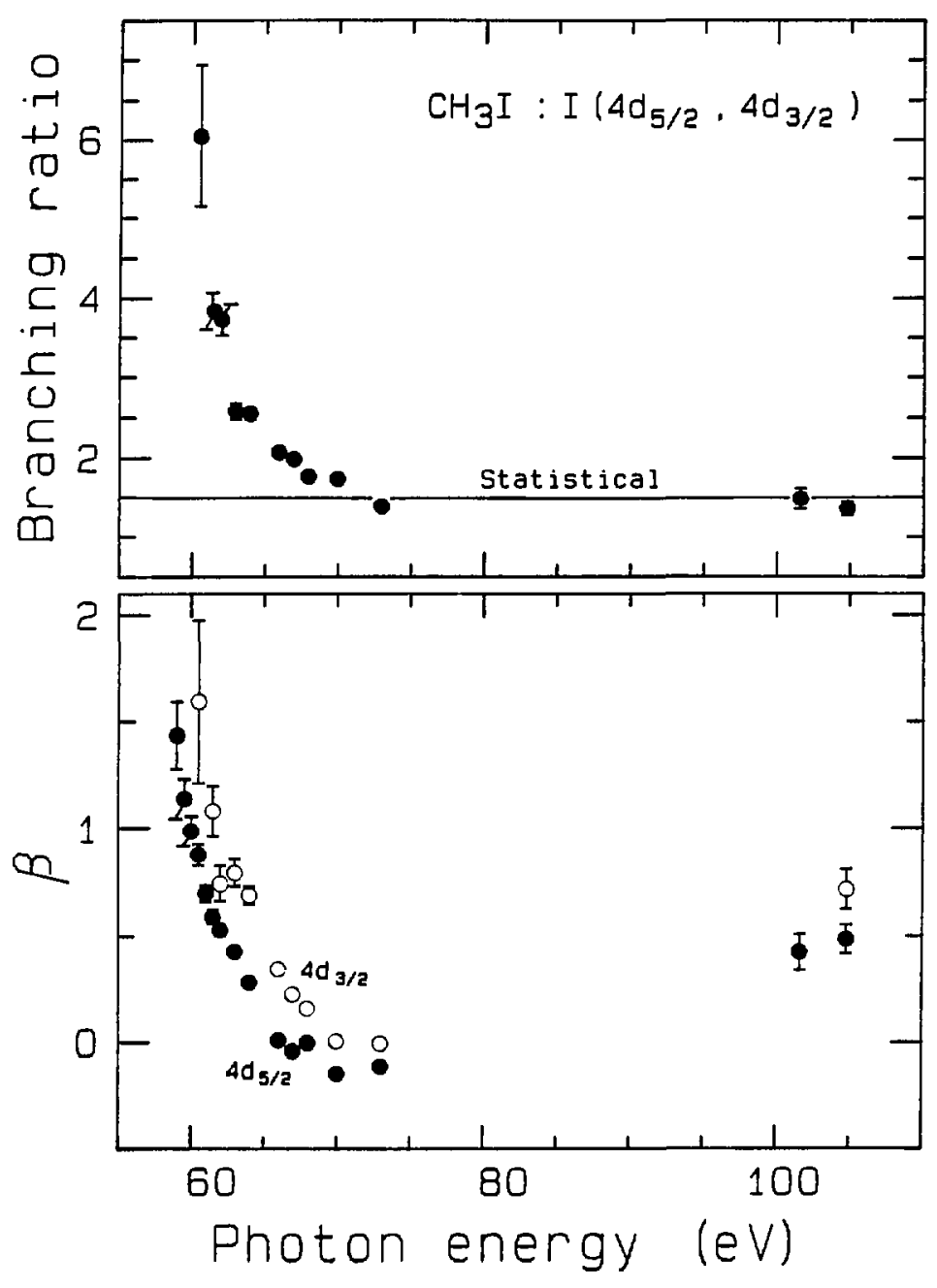

XBL 836-10194

Figure 4 
$-54-$

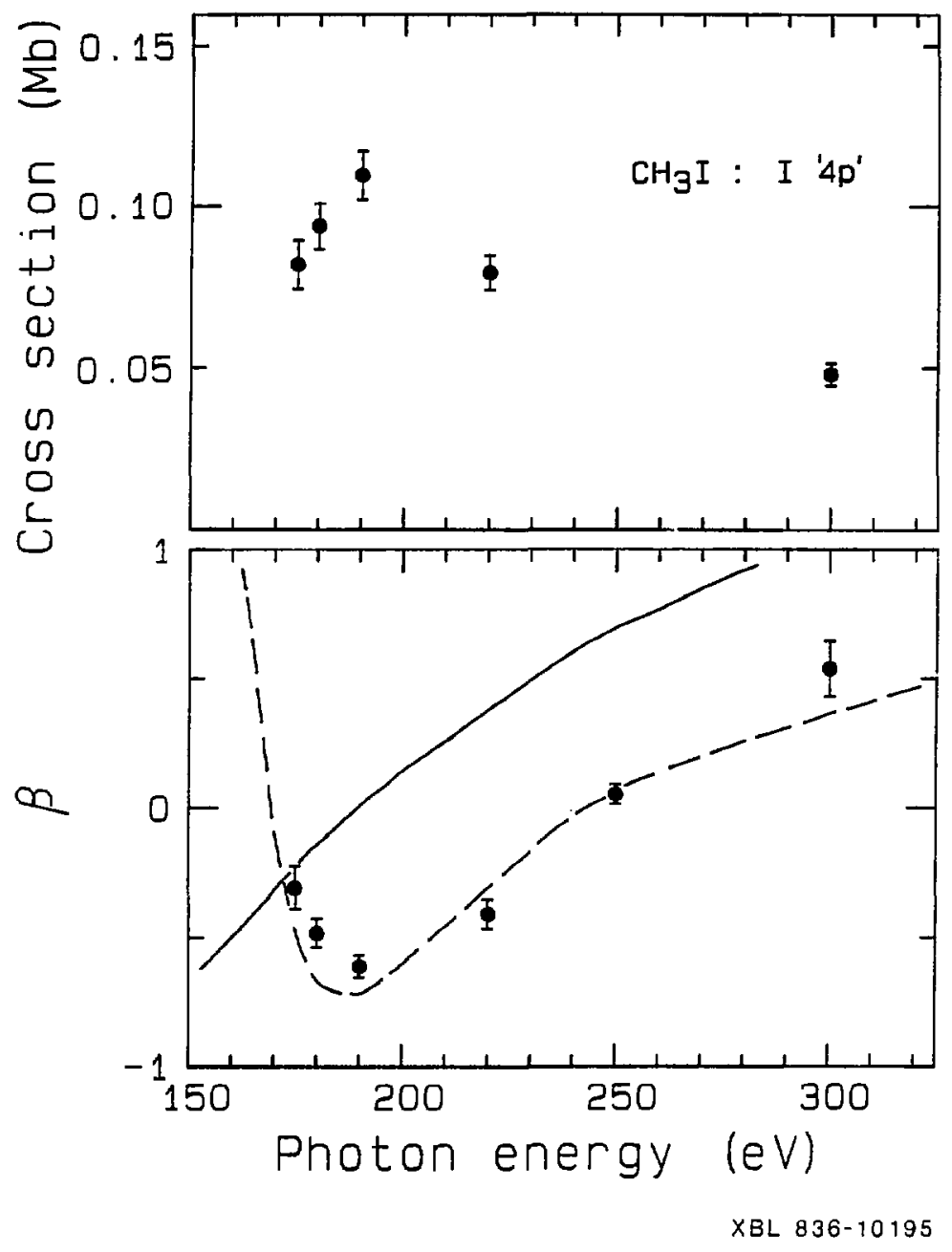

Figure 5 
V. NITROGEN K-SHELL PHOTOEMISSION AND AUGER EMISSION FROM N 2 AND NO*

The process of molecular core-level photoionization, al though initiated in localized regions near atomic nuclei, may exhibit intrinsically molecular effects because of the interaction of the photoelectron with the anisotropic molecular field. ${ }^{1-3}$ one category of such effects arises from the shape of the molecular potential experienced by the photoelectron as it traverses from the interior to the exterior of the molecule: these effects therefore are referred to as 'shape resonances'. 4 Typically, a barrier of $-1-2$ Ry is present in the potential, and it traps the photoelectron temporarily. This results in an enhancement of the photoionization cross section at an energy corresponding to the top of the barrier and a consequent change in the angular distribution of photoelectrons. ${ }^{1-7}$

The initial theoretical work on molecular shape resonances dealt with the $K$ shell of $N_{2},{ }^{4}$ and the $N_{2}$ molecule continues to be of general interest in the theoretical ${ }^{1-3,5-7}$ and experimental ${ }^{8-18}$ study of shape resonances. However, experimental results for molecular core levels (i.e. excluding valence and inner-valence levels) have been restricted to absorption $8,13,13,15,16$ and electron energy1 oss $9,10,12,14,17,18$ measurements, with the exception of three recent works that reported the use of photoelectron spectroscopy (PES) to study the $C$ and $0 \mathrm{~K}$ shells, ${ }^{19}$ the $S i L_{2,3}$ subshell, ${ }^{20}$ and the $\mathrm{Br} M_{4,5}$ subshe $11^{21}$ in a few small molecules. The advantage of PES is its ability to measure photoelectron angular distributions, which 
can be sensitive to the molecular potential and provide complementary information to a cross-section measurement. We report PES studies of the $\mathrm{N} \mathrm{K}$ shell in both $\mathrm{N}_{2}$ and $\mathrm{NO}$. In addition, we report here $\mathrm{N} \mathrm{KVV}$ Auger yields and angular distributions for $\mathrm{N}_{2}$ in the interesting shape-resonance region.

A TOF spectrum of $\mathrm{N}_{2}$ at $420 \mathrm{eV}$ is shown in Fig. 1. The valence photoelectrons and the Auger electrons from the decay of $N$ is vacancystates are unresolved in all of the spectra. The spectra of NO have the same general appearance as the $\mathrm{N}_{2}$ spectrum in Fig. 1 . The photon energy of the monochromator was calitrated by measuring an excitation spectrum of the $1 \sigma_{u} \rightarrow 1 \pi_{g}\left(2 \mathrm{p} \pi_{g}-\right.$ referred to as $\left.\pi^{*}\right)$ resonance in $\mathrm{N}_{2}$ at $400.8 \mathrm{eV} .^{15,16}$ This also indicated a photon resolution of $2.5 \mathrm{eV}$ for the $\mathrm{N}_{2}$ results and $3.5 \mathrm{eV}$ for the $\mathrm{NO}$ measurements.

Figure 2 shows the cross-section and asymmetry-parameter results for $N$ is photoemission from $N_{2}$. The $N$ ls threshold in $N_{2}$ is 409.9 ev. 22 The cross-section data are normalized to the absorption measurement of 8 ianconi et al. 15 at $431.5 \mathrm{ev}$. Overall agreement with the absorption data is good, but the contrast ratio of the shape-resonance maximum at $419 \mathrm{eV}$ is larger and the feature at $414 \mathrm{eV}$ assigned as double excitation ${ }^{12}$ is not as pronounced in the present data. The latter result is probably a consequence of the poorer photon resolution used in this work. Related electron energy-loss measurements ${ }^{12,14,17}$ compare similarly to the present data and agree very well with the absorption results. Also shown with the cross- 
section data are theoretical curves obtained with the multiplescattering method (MSM) ${ }^{4}$ and with Stieltjes-Tchebycheff moment theory (STMT). ${ }^{6}$ Both calculations correctly predict the presence of a shape resonance arising from a centrifugal barrier in the $1 \sigma_{g}+\mathrm{ko}_{\mathrm{u}}$ $\left(2=3^{5,6}\right)$ continuum channel. Quantitative agreement is better with the STMT calculation.

The $N$ is asymmetry parameter in $\mathrm{N}_{2}$, shown in the bottom of Fig. 2, is essentially constant from 414 to $418 \mathrm{eV}$, then rapidly increases to a high-energy asymptote near 2 by $440 \mathrm{eV}$. The proximity of the energy of the change in slope (418 ev) to the maximum in the cross section (4l9 eV) suggests that $\beta$ is affected by the shape resonance. Qualitatively, this is borne out in the MSM calculations of Dill et al. ${ }^{1}$ shown in the bottom of Fig. 2. Both the localized and delocalized curves indicate a minimum in a near the peak in the MSM curve for the cross section. However, the calculated changes are much laryer than that observed. This may indicate that ground-state nuclear-vibrational effects in $\mathrm{N}_{2}$ are significant for core-level shape-resonance phenomena. ${ }^{23}$ Above $422 \mathrm{eV}$, presumably past the shape-resonance region, both curves agree well with experiment. The predicted and observed approaches to the high-energy asymptote of $\beta=2$ show that $N 1$ photoemission from $N_{2}$ becomes nearly atomic-like at high photoelectron energies. All of the $N$ ls results exhibit behavior similar to the C 1 s measurements for the co molecule, ${ }^{19}$ which is isoelectronic with $\mathrm{N}_{2}$.

The results for the $\mathrm{N} \mathrm{KVV} \mathrm{Auger} \mathrm{peak} \mathrm{from} \mathrm{N}_{2}$ are shown in Fig. 
3. The data shown have been corrected for the valence photoelectron contribution that is unresolved from the Auger peak in the TOF spectra. The valence intensity and asymmetry parameter were estimated from spectra taken at photon energies below the discrete $\pi^{*}$ resonance, where only valence ionization is energetically allowed. These values were assumed constant over the energy range of the measurements. The cross-section data were corrected by subtracting a constant background of approximately $0.2 \mathrm{Mb}$. This procedure did not change substantially the appearance of the data. On the other hand, the asymmetry-parameter correction was greatest at energies for which the Auger yield was lowest. The absolute corrections to $\beta$ ranged from 0.0 to 0.3 , all to lower values because of the relatively large $\beta$ value $(0.8)$ for the valence photoelectrons in this energy range. Interpretation of the asymmetry-parameter results must be made in light of this variable, and substantial, correction to the data.

The $N$ KVV Auger yield from $\mathrm{N}_{2}$, shown in the top of $\mathrm{Fig.} \mathrm{3,} \mathrm{has}$ been normalized to the absorption measurement ${ }^{15}$ at $431.5 \mathrm{eV}$. Agreement with the absorption results is very good; somewhat tetter than the agreement of the $N 1 \mathrm{~s}$ data. The broadening effect of the larger monochromator bandpass used in the present measurements is apparent in the $\pi^{\star}$ resonance profile at $400.8 \mathrm{eV}$. The MSM ${ }^{4}$ and STMT $^{6}$ caiculations are shown also for comparison, and we again note the better predictive abilities of the STMT approach.

In discussing the Auger asymmetry-parameter results, it is helpful to consider the formalism developed by $0 i 11$ et al, ${ }^{24}$ They 
treat molecular K-shell photoionization and Auger decay as a two-step process in which the angular distribution of the subsequent Auger electrons depends explicitly on the alignment of the molecular ion. As a result, the Auger asymmetry parameter ${ }_{A}$ can be written

$$
B_{A}(h v)=C B_{m}(h v) \text {, }
$$

where ${ }_{m}(h v)$ is an orientation parameter dependent on the alignment produced by the initial photoionization event, and $c$ is a constant characteristic of each Auger decay channel. The values of $B_{m}\left(h_{v}\right)$ and $c$ are restricted to the ranges -1 to 2 , and $-1 / 2$ to 1 , respectively. Note that all of the photon-energy dependence is contained in $B_{m}\left(h_{v}\right)$.

The Auger asymmetry-parameter results in the bottom of Fig. 3 represent weighted averages over all of the unresolvea i ${ }_{2} \mathrm{~N}$ KVV transitions. 25,26 The $\theta_{A}$ results can be divided into three groups; the three points in the $\pi^{*}$ resonance region, the data near thresholo, and the results above threshold (> $414 \mathrm{ev}$ ). The last group has an average $B_{A}$ of $-0.05(4)$, consistent with an average value of zero within systematic error. These results are compared in Fig. 3 to $B_{m}(h v)$ from $0 i 11$ et al. ${ }^{24}$ The $B_{A}$ data do show a systematic curvature in this energy range, which may result from overestimation of the valence contribution to the measured asymetry parameter, because the estimate was made at lower photon energies where the valence cross section is larger. An explanation consistent with the calculated curve for $B_{m}\left(h_{v}\right)$ can be understood by noting that ${ }_{A}$ 
peaks at a photon energy of $-419 \mathrm{eV}$, slightly below the theoretical peak of $421 \mathrm{eV}$ for $B_{m}(h v)$. A shift in peak energy, together with a greater width and smaller contrast ratio of the experimental maximum, constituted the difference between experiment and theory for the $N$ is cross section. However, the experimental $\beta_{A}$ data would have to be shifted upward by about 0.2 in order for this "agreement" between $\beta_{A}$ and $\beta_{m}\left(h_{v}\right)$ to be meaningful, because $\beta_{A}$ and $\beta_{m}\left(h_{v}\right)$ should be related by the proportionality constant $C$ that is independent of photon energy. Further experiments with higher resolution would be desirable to clarify this situation.

Several possible explanations could reconcile the difference between the theoretical $B_{m}\left(h_{v}\right)$ and the experimental $B_{A}$. Because we measure $B_{A}$ values averaged over all of the Auger transitions, it is possible that the values of $c$ for the different Auger channels cause $\beta_{A}$ to average to near zero at all energies. Another possibility is that all or most of the $c$ parameters are near zero. Finally, the lack of agreement between the experimental and theoretical results may bring into question the treatment of Auger decay as a two-step process.

The $\beta_{A}$ data near threshold show values slightly lower than the results above threshold. Whether these values indicate Auger asymmetries, as described by Dill et al., ${ }^{24}$ is unclear. Near threshold, many Rydberg-like states are formed, but they are unresolved in our measurements.

The results at the $\pi^{*}$ resonance clearly indicate a nonzero value 
of $\beta_{A}$. While it is certain from adsorbed-molecule experiments ${ }^{27}$ and symmetry arguments that the $\pi^{*}$-excited $N_{2}$ molecule is strongly oriented, the asymetry of the autoionization electrons is quite small. The explanation ${ }^{19}$ is probably that the excited electron remains as a "spectator", only weakly coupled to the decay process, thus retaining the total orientation of the molecule in the $\pi^{*}$ orbital, while the subsequent electrons from an Auger-like decay show only an isotropic distribution, as would be required by angularmomentum conservation in the absence of strong coupling. The small, but nonzero, $\beta_{A}$ may be understood from the observation ${ }^{28}$ that a fraction of the autoionization decay from the $\pi *$ resonance in $\mathrm{N}_{2}$ yields final states in which the excited electron did take part in the decay process. Previous measurements on the Ne $K$ she $11^{29}$ and the $C$ $K$ shells of a few small molecules ${ }^{19}$ have yielded similar results.

The results for $N$ is photoemission from NO are presented in Fig. 4. There are two $N$ 1s thresholds at $410.3 \mathrm{eV}\left({ }^{3} \Pi\right)$ and $411.8\left({ }^{1} \pi\right) .{ }^{22}$ We adopt an average binding energy of $411 \mathrm{eV}$ for the unresolved $\mathrm{N}$ is peak in our work. Our cross-section results fren $N$ is photoemission have been scaled to the electron energy-loss measurements ${ }^{30}$ for best agreement. Both sets of data exhibit the same general behavior, but the present results do not extend to low enough energy to observe the shape-resonance maximum at $414 \mathrm{eV} .{ }^{30}$ Also shown is a MSM calculation ${ }^{31}$ which predicts the maximum 4-5 eV too high and a contrast ratio inuch too large.

The NO N 1s asymmetry-parameter res'ilts in Fig. 4 show that the $B$ 
is low near threshold and rises rapidly to 2 by $425 \mathrm{eV}$. The predicted minimum from the MSM calculations ${ }^{3}$ is not apparent. The observed shift of the measured $\beta_{1 \mathrm{~s}}$ values to lower energy by 4-5 eV with respect to the theoretical results is consistent with the shift observed for the $\mathrm{N}$ is cross-section maximum. 


\section{REFERENCES}

*hork done in collaboration with C.M. Truesdale, P.H. Kobrin, T.A. Ferrett, P.A. Heimann, U. Becker, H.G. Kerkhoff, and D.A. Shirley 1. D. Dill, S. Wallace, J. Siege1, and J.L. Dehmer, Phys. Rev. Lett. 41, 1230 (1978); 42,411 (1979).

2. J.L. Dehmer and D. Dill in Electron-Molecule and Photon-Molecule Collisions, ed. by T. Rescigno, V. Mckoy, and B. Schneider (Plenum Press, New York, 1979).

3. S. Wallace, Ph.0. Thesis, Boston University (unpublished).

4. J.L. Dehmer and D. Dill, Phys. Rev. Lett. 35, 213 (1975).

5. J.L. Dehmer and D. Di11, J. Chem. Phys. $\underline{65}, 5327$ (1976).

6. T.N. Rescigno and P.W. Langhoff, Chem. Phys. Lett. 51, 65 (1977).

7. P.W. Langhoff in Electron-Molecule and Photon-Molecule Collisions, ed. by T. Rescigno, V. Mckoy, and B. Schneider (Plenum Press, New York, 1979).

8. M. Nakamura, M. Sasanuma, 5. Sato, M. Watanabe, H. Yamashita, Y. Iguchi, A. Ejiri, S. Nakai, S. Yamaguchi, T. Sagawa, Y. Nakai, and T. Oshio, Phys. Rev. 178, 80 (1969).

9. M.J. Yan der Wiel, Th.M El-Sherbini, and C.E. Brion, Chem. Phys. Lett. I, 161 (1970).

10. M.J. Van der Wiel, and Th.M. El-Sherbini, Physica 59, 453 (1972).

11. A.S. Vinogradov, B. SchTarbaum, and T.M. Zimkina, Opt. Spectrosc. 36, 383 (1974); [0pt. Spektrosk. 36, 658 (1974)].

12. G.R. Wight, C.E. Brion, and M.J. Van der Wiel, J. Electron Spectrose. 1, 457 (1973). 
13. A.S. Vinogradov, T.M. Zimkina, V.N. Akimov, and B. Schlarbaum, Izv. Akad. Nauk. SSSR, Fiz. Ser. 38,508 (1974).

14. R.B. Kay, Ph.E. Van der Leeuw, and M.J. Van der Wiel, J. Phys, B 10, 2513 (1977).

15. A. Bianconi, H. Petersen, F.C. Brown, and R.Z. Bachrach, Phys. Rev. A 17, 1907 (1978).

16. H. Petersen, A. Bianconi, F.C. Brown, and R.Z. Bachrach, Chem. Phys. Lett. 58, 263 (1978).

17. A.P. Hitchcock and C.E. Brion, J. Electron Spectrose. 18, 1 (1980).

18. M. Tronc, G.C. King, and F.H. Read, J. Phys. B 13, 999 (1980).

19. C.M. Truesdale, S.H. Southworth, P.H. Kobrin, U. Becker, D.W. Lindle, H.G. Kerkhoff, and D.A. Shirley, Phys. Rev. Lett. 50, 1265 (1983); C.M. Truesdale, D.W. Lindle, P.H. Kobrin, U.E. Becker, H.G. Kerkhoff, P.A. Heimann, T.A. Ferrett, and D.A. Shirley, J. Chem. Phys. (in press).

20. P.R. Keller, J.W. Taylor, F.A. Grimm, P. Senn, T.A. Carlson, and M.0. Krause, Chem. Phys. 74,247 (1983).

21. T.A. Carlson, M.O. Krause, F.A. Grim, P.R. Keller, and J.W. Taylor, Chem. Phys. Lett. 87, 552 (1982).

22. K. Siegbahn, C. Nordling, G. Johansson, J. Hedman, P.F. Hedén, K. Hamrin, U. Gelius, T. Bergmark, L.0.Werme, R. Manne, and Y. Baer, ESCA Applied to Free Molecules (North-Holland, Amsterdam, 1969). 23. J.L. Dehmer, D. Dill, and S. Wallace, Phys. Rev. Lett. $\underline{43}, 1005$ (1979).

24. D. Dill, J.R. Swanson, S. Wallace, and J.L. Dehmer, Phys. Rev. 
Lett. $45,1393(1980)$.

25. T.A. Carison, W.E. Moddeman, B.P. Pullen, and M.0. Krause, Chem. Phys. Lett. $\underline{5}, 390$ (1970).

26. W.E. Moddeman, T.A. Carlson, M.0. Krause, B.P. Pullen, W.E. Bull, and G.K. Schweitzer, J. Chem. Phys. 55, 2317 (1971).

27. J. Stöhr and R. Jaeger, Phys. Rev. $8 \underline{26}, 4111$ (1982).

28. T.D. Thomas (private communication).

29. P.H. Kobrin, S. Southworth, C.M. Truesdale, D.W. Lindle, U. Becker, and D.A. Shirley, Phys. Rev. A 29 (1984).

30. G.R. Wight and C.E. Brion, J. Electron Spectrosc. 4, 313 (1974).

31. S. Wallace, D. Dill, and J.L Dehmer, J. Chem. Phys. 76, 1217 (1982). 


\section{IGURE CAPTIONS}

Fig. 1. TOF photoelectron spectrum of $\mathrm{N}_{2}$ at $420 \mathrm{eV}$ photon energy taken with the $0^{\circ}$ detector. The high-energy peak includes both N KVV Auger electrons and valence photoelectrons.

Fig. 2. Cross-section (top) and asymmetry-parameter (bottom) results for $N$ is photoemission from $\mathrm{N}_{2}$. The cross-section data have been scaled to the absorption result (solid curve), Ref. 15, at $431.5 \mathrm{eV}$. Theoretical curves; top panel - MSM (short-dashed), Ref. 4, STMT (long-dashed), Ref. 6; bottom panel - localized (solid) and delocalized (dashed) MSM calculations, Ref. 1.

Fig. 3. Yield (top) and asymetry parameter (bottom) for N KVV Auger emission from $\mathrm{N}_{2}$. The threshold for $\mathrm{N}$ 1s photoemission $(409.9 \mathrm{eV})$ has been indicated in both panels. The Auger-yield data have been scaled as in Fig. 2. All of the cross-section results above $405 \mathrm{eV}$ have been multiplied by 5 . The theoretical curves in the top panel are the same as those in the top panel of Fig. 2. In the bottom pane1, the solid curve is a calculation of $\beta_{m}\left(h_{v}\right)$ using the MSM, Ref. 24.

Fig. 4. Cross-section (top) and asymmetry-parameter (bottom) results for $N$ is photoemission from NO. The cross-section data have been scaled to the electron energy-loss results (solid curve), Ref. 30, for best agreement, and both are in arbitrary units. The dashed curve in the top panel is the MSM result, Ret. 31, in Mb. The solid curve in the bottom panel is an MSM calculation, Ref. 3. 


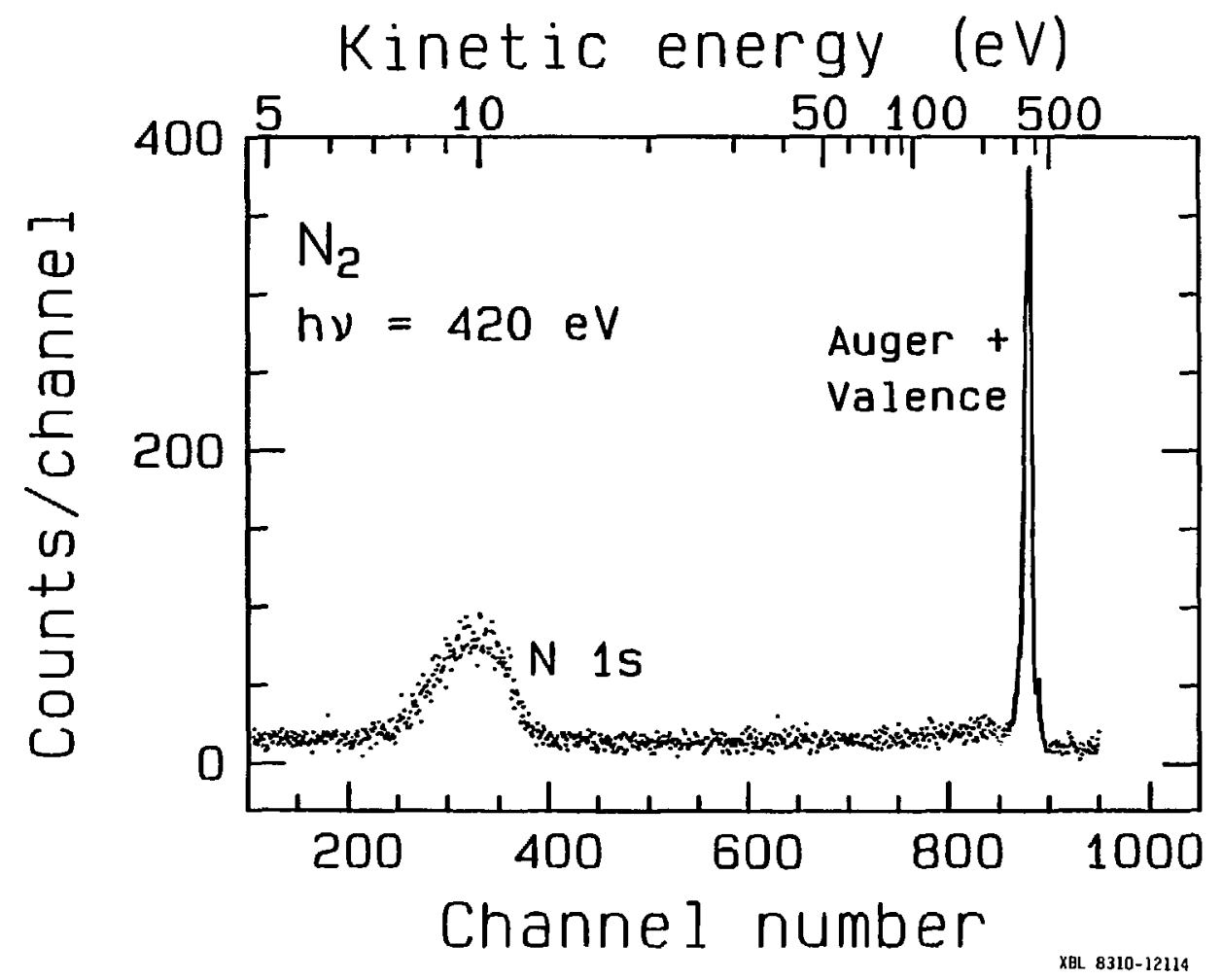

Figure 1 
$-68-$
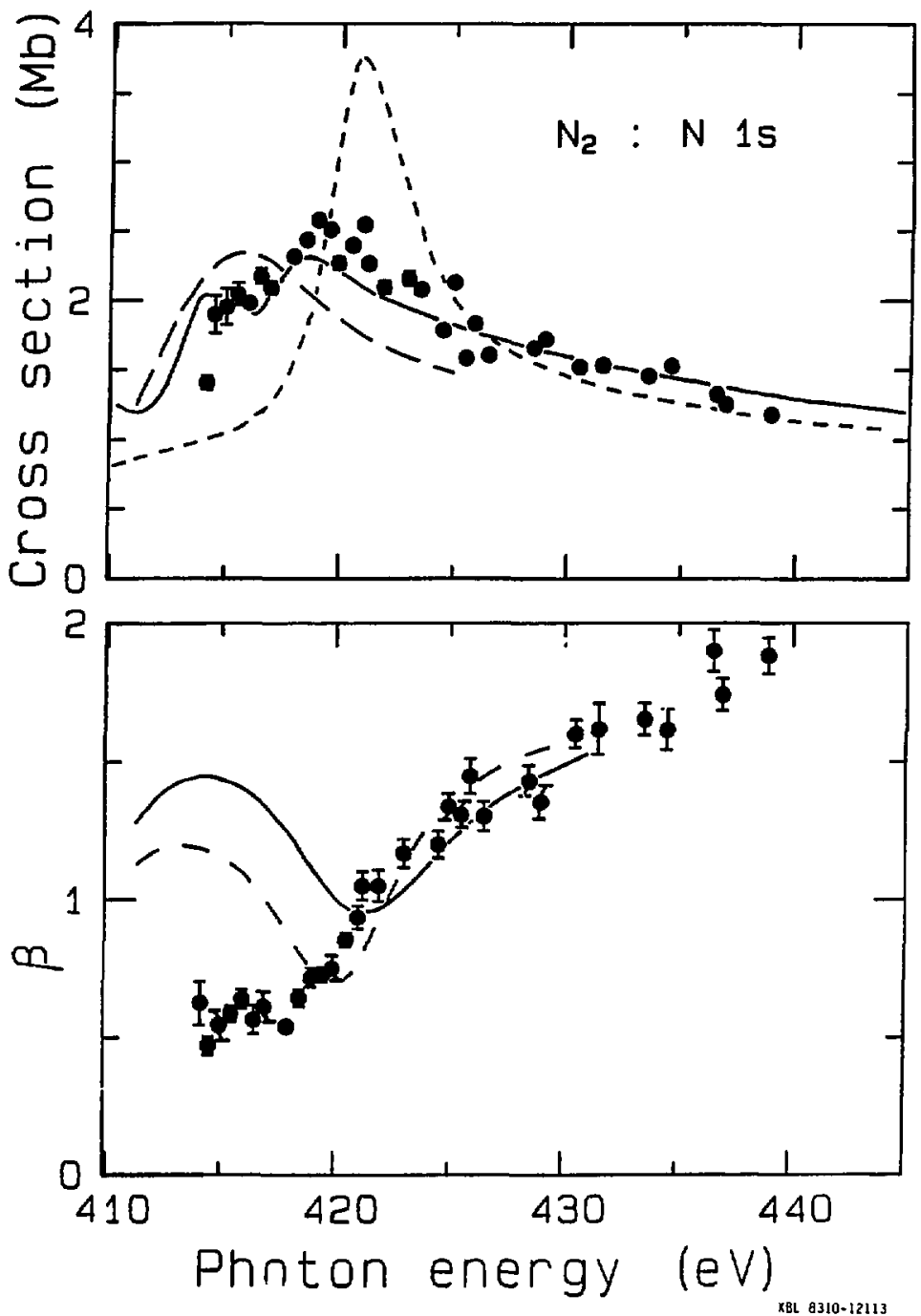

Figure 2 
$-69$.

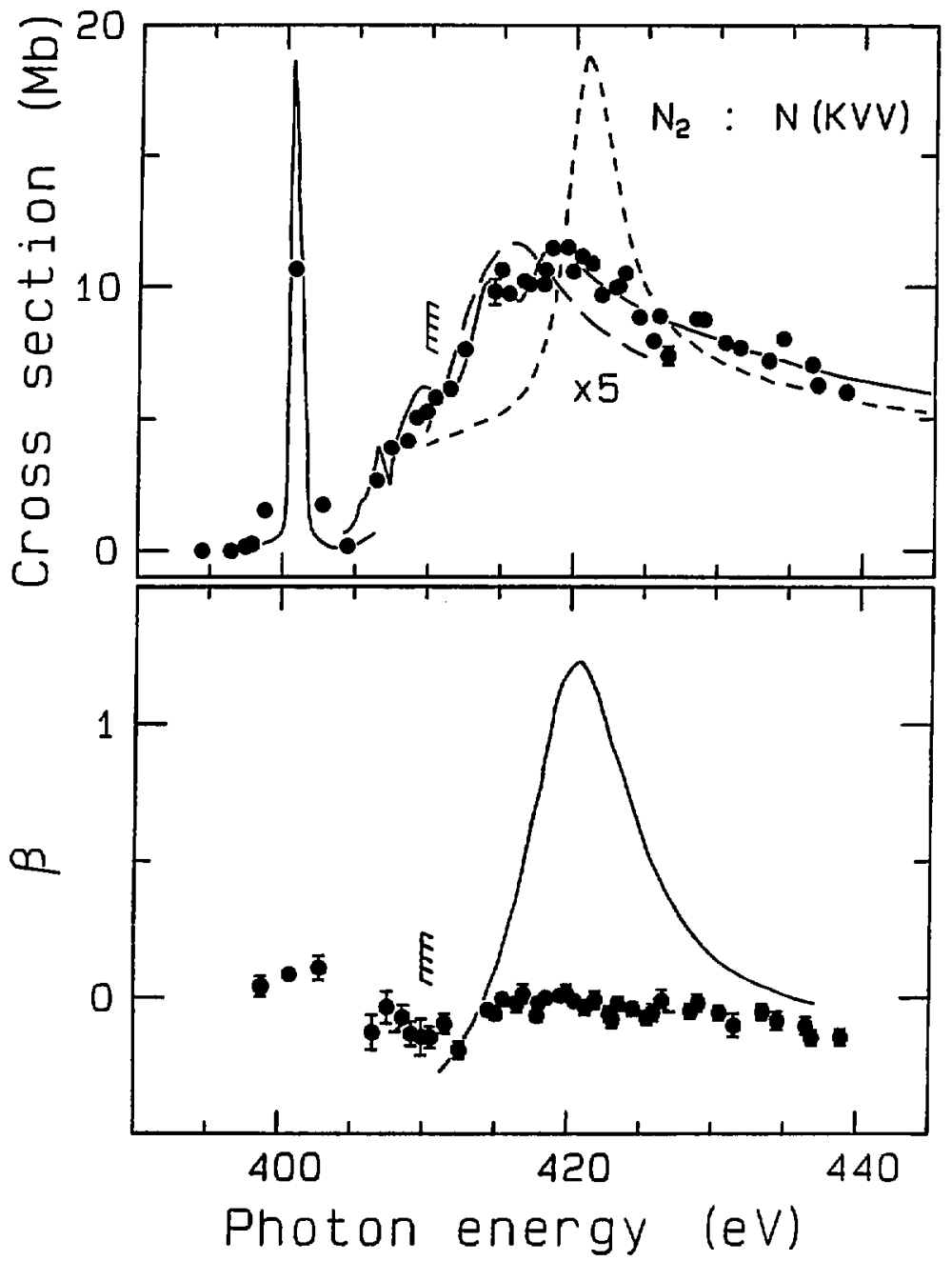

Xat 8310-12115

Figure 3 


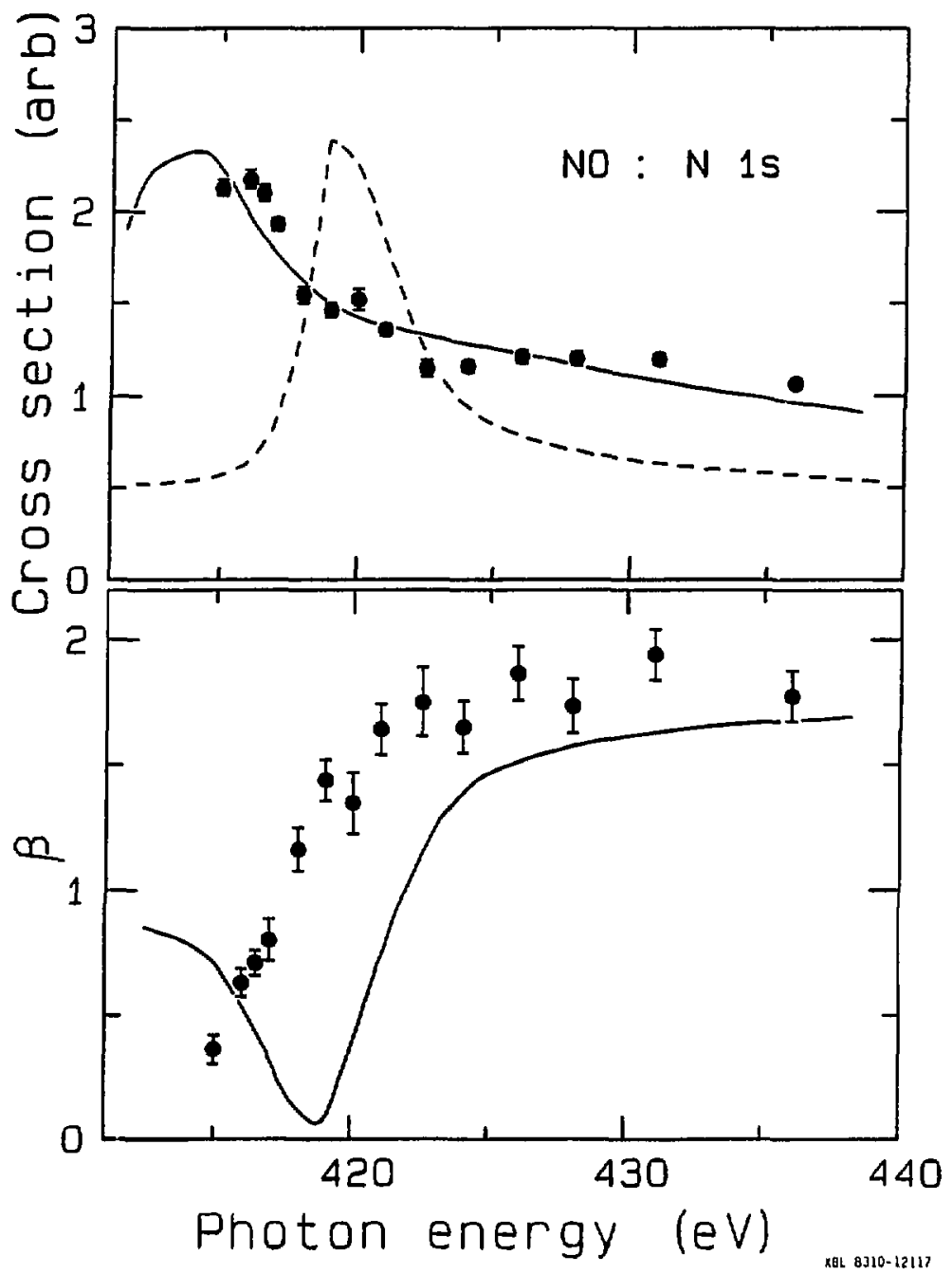

Figure 4 
VI. PHOTOIONIZATION OF HELIUM ABOVE THE $\mathrm{He}^{+}(n=2)$ THRESHOLD: AUTOIONIZATION AND FINAL-STATE SYMMETRY*

\section{A. Introduction}

The photoionization of helium provides tine simplest example of electran correlation in atomic physics. Because correlation cannot occur in the hydrogen-like final state, theoretical studies of initialstate and continuum-state correlation effects are easier to interpret for the photoionization process. For this reason, helium is an important system for testing various theoretical approaches to the phenomenon of electron correlation. Past interest focused upon absolute cross-section mec surements, as reviewed by Marr and West. ${ }^{1}$ Theoretical calculations ${ }^{2}$ of the total cross section, at least below the $\mathrm{He}^{+}(\mathrm{n}=2)$ threshold, have proven to be very accurate. Recent interest $^{3-12}$ has centered upon the photoionization processes above this threshold, which can leave the residual $\mathrm{He}^{+}$ion in the $2 \mathrm{~s}$ or $2 \mathrm{p}$ excited states. Several calculations ${ }^{13-19}$ of the partial photoionization cross section, the satellite and subshell branching ratios, and the angular-distribution asymetry parameter have been performed for the $\mathrm{He}^{+}(\mathrm{n}=2)$ states, indicating that much is still to be learned from this simple system about the influence of correlation on measurable quantities.

Figure 1 depicts the atomic and ionic states in helium relevant to this experiment. We have studied the photoionization processes 


$$
H e\left(1 s^{2}\right)+h v \rightarrow H e^{+}(1 s, 2 s, 2 p)+e^{-}
$$

as a functicn of photon energy and photoelectron ejection angle. The $\mathrm{He}^{+}(2 \mathrm{~s})$ and $\mathrm{He}^{+}(2 \mathrm{p})$ states are effectively degenerate in a photoemission experiment, and thus comprise a single satellite line, which we designate $\mathrm{He}^{+}(\mathrm{n}=2)$. We have measured the partial cross $\mathrm{sec}^{+}$ions, $a$, and angular-distribution asymmetry parameters, $B$, for the processes represented in Eq. (1) that leave the $\mathrm{He}^{+}$ion in the 1 or the $\mathrm{n}=2$ final states, as well as the branching ratio, $R_{21}=\sigma_{n=2} / \sigma_{1 s}$, of the satellite intensity relative to that of the main line. The threshold for production of the il=2 states from the ground state of the helium atom is $65.4 \mathrm{eV}$. We have taken photoelectron spectra for photon energies from $1.9 \mathrm{eV}$ above this threshold to $90 \mathrm{eV}$. This energy range can be divided into resonance and nonresonance regions. In the resonance region, it is possible to excite a series of Rydberg levels leading to the third ionization threshold at $73.0 \mathrm{eV}$, with subsequent autoionization.

The nonresunanes data, taken with photon energies in the ranges 67.5 to $69.5 \mathrm{eV}$ and 75 to $90 \mathrm{eV}$, show good agreement with previous measurements $4,6-8,10,12$ and calculations ${ }^{13-19}$ of the partial cross sections for both the $n=1$ and $n=2$ final states (processes DI in Fig. 1) and for the total (i.e. 2s plus 2p) satellite branching ratio. The ratin of the $2 p$ cross section to the $2 s$ cross section, $R$, which can be derived from $\beta_{n=?}$, provides a more sensitive test of theory. Discrepancies exist among the various experimenta $1^{9-12}$ and 
theoretical ${ }^{14-19}$ values of $R$ reported to date. Chang ${ }^{16}$ has predicted that near threshold the 2 contribution to $\sigma_{n=2}$ is larger than that of the $2 p$ level, in contrast to several other predictions. 14,15,17-19 Experimentally, the earliest photoemission data ${ }^{9}$ were consistent with either calculation, but later photoemission 11,12 and fluorescence ${ }^{10}$ measurements tended to discount Chang's prediction. Our results support the conclusion that the $\mathrm{He}^{+}(n=2)$ final state is predominantly $2 \mathrm{p}$ near threshold. The disagreentent in the theoretical results indicates the need for a bette understanding of the contributions of electron correlation to the photoionization process.

A different perspective on correlation can be obtained in the analysis of autoionization resonances. The interaction of various continuum-state wavefunctions with an excited Rydberg level determines the extent and profile of the autoionization process. We have taken photoelectron spectra in the region of the lowest four Rydberg levels leading to the $\mathrm{He}^{+}(n=3)$ ionization threshold at $73.0 \mathrm{eV}$. The four levels studied are indicated in the left-hand portion of Fig. 1. Large variations in $\sigma_{n=2}$ and $\beta_{n=2}$ were found at these resonances. Our measured variation in $\sigma_{n=2}$ agrees with the fluorescence data of Wondruff and Samson. 10 The detailed variation of ${ }_{n=2}$ over the autoionization resonances is presented here for the first time. In addition, we infer from our measurements that the shape of the total cross section over the first member of this Rydberg series differs with earlier experimental 20 and theoretical ${ }^{21,22}$ results, but is 
consistent with recent photoemission data. 12

An example of a time-of-flight spectrum for helium from the $54.7^{\circ}$ detector is shown in Fig. 2. The nonresonance data are presented in Section $B$, and the behavior of the cross sections, branching ratios and asymmetry parameters over the autoionization resonances is discussed in Section C. Conclusions are presented in Section D.

\section{B. NONRESONANCE PHOTOIONIZATION}

Our measurement of the branching racio $R_{21}$ is shown in Fig. 3 along with several theoretical curves and other experimental measurements. The present results show excellent agreement with the previous measurements. $4: 5,8$ We see also that the available theoretical calculations ${ }^{13-17}$ agree reasonably well with one another and predict the branching ratio to within $-25 \%$. The absolute partial cross section of the $n=2$ satellite is also predicted well by these same calculations. We do not show our relative partial cross sections for the $n=2$ or $1 \mathrm{~s}$ levels, but note that they also agree with the previous measurements.

The ability to predict the energy dependence of $R_{21}$ appears to be nearly independent of the degree of configuration interaction (CI) included in the calculations, tut this is not true when the individual partial cross sections for the $2 \mathrm{~s}$ and $2 \mathrm{p}$ final states are considered. The measurement of these partial cross sections, or their ratio, is a more sensitive test of the theoretical calculations, because the energy dependences of $\sigma_{2 s}$ and $\sigma_{2 p}$ are quite different. The reasons 
for these differences can be understood from a discussion of CI as it pertains to helium photoionization.

For the case that $\mathrm{CI}$ is only important in the initial (eigen) state of the helium atom (ISCI), the initial state is written properly as an admixture of the $1 \mathrm{~s}^{2}, 1 \mathrm{~s} 2 \mathrm{~s}, 2 \mathrm{~s}^{2}, 2 \mathrm{p}^{2}$, and higher configurations. The ground state is predominantly $1 s^{2}$. If we consider then that the $1 s^{2}, 1 s 2 s, 2 s^{2}, 2 p^{2}$, etc. configurations are not eigenstates (i.e. they are not orthogonal to each other), then the degree to which any one of the other configurations mixes into the ground state can be estimated by considering its overlap with the $1 \mathrm{~s}^{2}$ configuration. ${ }^{23}$ This estimate suggests that $1 \mathrm{~s} 2 \mathrm{~s}$ and $2 \mathrm{~s}^{2}$ play a more important role in ISCI than does $2 p^{2}$. Furthermore, if we assume that the partial cross sections for the production of the $2 s$ and $2 p$ final states (by ionization from the $1 s 2 s, 2 s^{2}$, or $2 p^{2}$ configurations) are dependent only on the mixing coefficients of $1 s 2 s, 2 s^{2}$, and $2 p^{2}$ in the initial state, then we expect ISCI to lead to a predominance of $\mathrm{He}^{+}(2 \mathrm{~s})$. We also can see that this result is valid for all photoelectron energies because the ISCI mixing coefficients are independent of energy. Thus, if CI were to occur only in the initial state, the ratio $R$ would be constant and probably less than unity. However, the effects of $\mathrm{CI}$ in the continuum state (CSCI) must also be considered. The continuum state includes both the ionic core left after photoionization and the photoelectron. For simplicity in this discussion, we will consider the initial state to be completely $1 s^{2}$. Configuration interaction in the continuum thus constitutes 
mixing of configurations such as 2 sep and 2pes with 1sep. Again, we estimate the degree of mixing by examining the overlap of a given configuration with 1sep. Because the photoelectron is included in these wavefunctions, we can expect the mixing coefficients, and hence the $2 \mathrm{~s}$ and $2 p$ cross sections, to be energy dependent. Because of the nature of continuum wavefunctions at low energy, the overlap between 2pes and 1sep is largest near threshold, diminishing with increasing eniergy. $^{23}$ The overlap between 2 sep and 1 sep will be less energy dependent, because of the similarity of the continuum part of these two configurations. The result of CSCI would then likely be a predominance of the $2 p$ final state near threshold, this predominance decreasing as the energy increases. This discussion of rSCI is oversimplified to the extent that we have ignored the near degeneracy of the $2 \mathrm{~s}$ and $2 p$ final states. Their proximity in energy allows them to be strongly coupled in the continuum state. Despite this complication, we expect the conclusions to be qualitatively correct. 23

To understand the complete picture for helium photoionization, it is necessary to combine the effects of ISCI and CSCI discussed above. Configuration interaction in the hydrogen-like final state is not possible, and does not need to be considered. Dqualitatively, we conclude that at threshold the $2 p$ final state will be maximally produced and may be more likely than the $2 s$ firal state, whereas in the high-energy limit, the $2 s$ final state wili be most important. Similar conclusions can be derived fron. . different argument using shake theory. 17 Quantitatively, the degree of predominance of one 
final state over the other and the applicable energy ranges can only be determined by theoretical calculations that accurately treat the effects of ISCI and CSCI. Comparison of such calculations with experimental results can yield information about the type and degree of $\mathrm{Cl}$ present in the helium system, as well as important results concerning what treatments of $\mathrm{CI}$ are most accurate.

Experimentally, two approaches have been taken. Woodruff and Samson ${ }^{10}$ measured $R$ directly by taking advantage of the long rifetime of $\mathrm{He}^{+}(2 \mathrm{~s})$ to distinguish between fluorescence from the $2 \mathrm{~s}$ and $2 p$ levels. A second method, used by several groups, $9,11,12$ including ours, relies on the measurement of the asymetry parameter, $\theta_{n=2}$, which is a weighted average of $\theta_{2 s}$ and $\theta_{2 p}$ :

$$
B_{n=2}=\frac{\sigma_{2 s^{\beta} 2 s}+\sigma_{2 p}{ }^{\beta} 2 p}{\sigma_{2 s}+\sigma_{2 p}} .
$$

By rearranging Eq. (2), and assuming that $B_{2 s}$ is aiways $2, R$ can be expressed in termis of $B_{n=2}$ :

$$
R=\frac{a_{2 p}}{a_{2 s}}=\frac{2-\beta_{n=2}}{\theta_{n=2}-\sigma_{2 p}} \text {. }
$$

This latter approach of course, must rely on the calculated values of $\theta_{2 p}$. Fortunately, the two available calculations ${ }^{9,15}$ of $\beta_{2 p}$ agree rather well.

Our results for $\theta_{n=2}$ are presented in Fig. 4 along with the other experimental results $9,11,12$ for $\beta_{n=2}$ and theoretical 
calculations ${ }^{9,15}$ of $\beta_{n=2}$ and $\beta_{2 p}$. Agreement among the experiments is excellent throughout the energy range shown. The region between 70 and $75 \mathrm{eV}$ is blank because of the presence of several strong autoionizing resonances (see Section $C$ ). Figure 5 shows the values of $R$ derived from the experimental data in Fig. 4 and the calculated values of $\beta_{2 p}$ from Jacobs and Burke. ${ }^{15}$ Also shown are five calculations ${ }^{14-19}$ of this ratio and the direct measurements of $R$ by Woodruff and Samson.

The strong energy dependence of $R$ below $100 \mathrm{eV}$ can be understood from the above discussion of ISCI and CSCI. Because CSCI is more important at lower energies, we expect $\sigma_{2 p}$ to make a stronger contribution to $\sigma_{n=2}$ near threshold, as confirmed by the data. Also, since ISCI is nearly energy-independent, we expect the ratio $\sigma_{2 s} / \sigma_{1 s}$ to be fairly constant for all photon energies. Direct measurements ${ }^{10}$ of $a_{2 s}$ support this conclusion for the first $60 \mathrm{eV}$ above the $2 \mathrm{~s}$ threshold. At the high-energy limit the $n=2$ peak should be mostly $2 \mathrm{~s},{ }^{23}$ though no experimental confirmation is available. The sateliite branching ratio, $R_{21}$, in this limit has been calculated ${ }^{13,15}$ to be $4.8 \%$, which can be compared to the values of $6(1) \%$ at $190^{6}$ and $278 \mathrm{eV}^{3}$ photon energy and $5.0(8) \%$ for an $\mathrm{Al} \mathrm{Ka}$ measurement. 5

The theories used in the calculation of $R$ differ significantly in sophistication. The calculation of Richards and Larkins ${ }^{17,19}$ used Hartree-Fock (HF) wavefunctions in which the effects of relaxation have been included, but not $\mathrm{CI}$. The calculation by Chang ${ }^{16}$ also 
used HF wavefunctions, but included $\mathrm{CI}$ in both the initial state and the continuum states. Jacobs ${ }^{14}$ and Jacobs and Burke ${ }^{15}$ used a 56-term Hylleras initial-state wavefunction, which is a nearly exact approximation of the $\mathrm{He}\left(1 \mathrm{~s}^{2}\right)$ ground state, and a close-coupling calculation for the final state, which is similar to CSCI. The calculation by Berrington et al. ${ }^{18}$ is an improvement on the earlier close-coupling calculations in which care was taken to use the same configurations in the expansions for both the initial and continuum states. All of the calculations used final-state wavefunctions that are purely hydrogenic.

Above $85 \mathrm{eV}$, all of the calculations show the same behavior and they all agree with experiment to within the errors. Below $85 \mathrm{ev}$, however, Chang's curve deviates significantly from the rest and predicts a predominance of $2 \mathrm{~s}$ near threshold. The experimental results show clearly that this is not correct, and we conclude that the $2 p$ final state is the major component of $\mathrm{He}^{+}(n=2)$ at threshold, being more than twice as likely as the $2 \mathrm{~s}$ final state. The calculation of Berrington et al. Shows the best overall agreement with the present results.

The three close-coupling calcuiations (Jacobs, Jacobs and Burke and Berrington et al.) and the calculation of Richards and Larkins behave similarly at low energy, despite the fact that the latter is a much less sophisticated calculation. Richards and Larkins have concluded that $\mathrm{CI}$ and exchange are unimportant to their calculation except near threshold, and they cite this as the reason their 
calculation does so we11. Furthermore, they suggest ${ }^{24}$ that the discrepancy in Chang's calculation may be the result of the use of CI wavefunctions that do not accurately take into account the direct interaction of the outgoing channels, which the other calculations do. 14,15,18 This ultimately may be the result of cancellation between large terms in the calculation of the transition amplitude. ${ }^{16}$

This example illustrates that much is still to be learned about the effects of CI on the calculation of the energy dependence of satellite cross sections. Except for isolated calculations on the $\mathrm{Ne}$ $K$ shell, 25 the Li $K$ shel1, 17,19 and the valence shell of $\mathrm{Fe},{ }^{26}$ the helium calculations are the only such theoretical studies completed to date. Helium thus appears to be a good candidate for further studies of electron-correlation effects in atomic photoionization.

At the photon energy of $80 \mathrm{eV}$ (see fig. 2), we were able to detect photoelectrons from the $\mathrm{He}^{+}(\mathrm{n}=3)$ final state. We determine its branching ratio relative to the main line, $R_{31}$, to be $1.8(2) \%$ and its asymmetry parameter to be $-0.2(2)$. This value for $R_{31}$ is in agreement with previous estimates $8,17,27$ at this energy and the data of Bizau et al. 28 It also can be compared to the Al Ka result of $1.4(8) \% 5$ The negative value for $\theta_{n=3}$ indicates that the $3 s$ final state is not the major component of this peak. In fact, the result of $-0.2(2)$ is consistent with the threshold value of -0.04 calculated by Greene, 29 who found approximately equal contributions from 3s, 3p, and $3 d .30$ 


\section{RESONANCE PHOTOIONIZATION}

Madden and Codling ${ }^{31}$ first observed the Rydberg levels leading to the $n=3$ ionization threshold in helium in the energy range $69.5 \leq h_{\nu} \leq 73.0 \mathrm{eV}$. Of the five possible Rydberg series in this region, only one has been found to be significant. It is designated $\left(s p, 3 n^{+}\right) 1_{p^{*}}$, which is a positive admixture of $3 \operatorname{snp} 1_{p^{*}}$ and 3pns $1_{p^{*}}$. It has also been given ${ }^{32}$ the notation $1_{n}$, which we shall use here except when the $1_{3}$ resonance is discussed alone: then we shall refer to it as the $3 s 3 p$ resonance. The $(s p, 3 n-) 1_{p}^{*}$ (or $-1_{n}$ ) series, the negative admixture of 3 snp $1_{p^{\bullet}}$ and $3 p n s 1_{p^{\bullet}}$, has also been observed, but it is small enough to be neglected in the present analysis. The other possible ${ }^{1} P^{\bullet}$ series (with major component 3pnd, 3dnp or 3dnf) have not been observed.

The remainder of this section will deal with the analysis of the cross-section, branching-ratio, and asymmetry-parameter profiles over the $I_{n}$ series of resonances. To lay the groundwork for this analysis, we begin (Sect. C1) with a summary of several formalisms for describing autoionization phenomena. How these formalisms are used to fit the satellite data is discussed in Sect. C2. Quantitative results for the cross section and branching ratio of the $n=2$ satelite are presented in Sect C3. From these results, we have inferred qualitative information about the resonance profiles of $\sigma_{1 s}$ and the total cross section, $\sigma_{t}$. Resonance parameters are presented for $\sigma_{1 s}$ and $\sigma_{t}$ with the proviso that the quantitative results may not be very 
accurate, but are given to illustrate the usefulness of this type of analysis. To this end, we determine several other parameters, including individual dipole matrix elements, to iliustrate methods for the quantitative interpretation of an autoionization process. In Sect. 64 , the $B_{n=2}$ resonance profile is discl,sed quantitatively, and inferences for the ratio $R$ in the resonance region are made.

\section{THEORETICAL BACKGROUND}

The effect of an isolated resonance, such as a Rydberg level, on the total photoabsorption or total photoionization cross section was derived originally by $F$ ano. 33 The presence of a discrete leve] embedded in one or more continua causes an interference in the photon absorption process because of the indistinguishability of the two pathways, direct ionization and autoionization, leading to the final state. Fano derived the following expression for the total cross section, $\sigma_{t}$, for the case of a single discrete state interacting with one or more continuum states:

$$
\begin{aligned}
& \sigma_{t}=\sigma_{0}\left[\rho^{2} \frac{(q+c)^{2}}{1+c^{2}}+1-\rho^{2}\right], \\
& c=\frac{E-E_{0}}{\Gamma / 2},
\end{aligned}
$$

Where the Fano parameters $q$ and $\rho^{2}$ are assumed constant over the 
resonance, $\sigma_{0}$ is the cross section $f a r$ from the resonance, $\Gamma$ and $E_{0}$ are the full-width at half maximum (FWHM) and the position of the resonance, respectively, and $c$ is a reduced energy.

The quantities $q, p^{2}, \sigma_{0}$ and $\Gamma$ all can be expressed in terms of the dipole matrix elements for transitions from the ground state, 9 , ti the discrete state, $\phi$, and to the continua, $\mu$, together with the Coulomb-interaction matrix elements coupling the discrete state to the continua. The $\mathbf{q}$ parameter, which governs the shape of the total cross section, is given by

$$
q=\frac{\langle(|\vec{r}| g\rangle}{\pi \sum_{\mu}(\phi|V| \mu)(\mu|\vec{r}| g\rangle} \text {, }
$$

and the correlation coefficient, $p^{2}$, which is a measure of the strength of the resonance, is given by

$$
\rho^{2}=\frac{\sum_{\mu}|(\phi|V| \mu k \mu|\vec{r}| g\rangle|^{2}}{\sum_{\mu}|(\phi|V| \mu)|^{2} \sum_{\mu}|\langle\mu|\vec{r}| g\rangle|^{2}},
$$

where $\overrightarrow{\vec{r}}$ and $V$ represent the dipole and Coulomb operators, respectively, and $\theta$ is the discrete state modified by an admixture of the continuum states. The degree to which $\phi$ is different from $\phi$ is dependent upon the energj' yariations of the continuum wavefunctions in the vicinity of the resonance. The linewidth of the resonance is given by 


$$
I=2 \pi \sum_{\mu}|(\phi|v| \mu)|^{2},
$$

and the nonresonance, background cross section is given by

$$
\left.\sigma_{0}=\sum_{\mu}\left|k_{\mu}\right| \vec{r} \mid g\right)\left.\right|^{2}
$$

While the matrix elements in Eqs. (5) and (6) are not strictly energyindependent, they are slowly-varying functions of energy, and $q$ and $p^{2}$ are therefore assumed to be constant in the vicinity of the resonance.

The Fano parametrization [Eys. (4)-(8)] can explain the many different shapes measured for autoionization resonances. For example, the sign of the $q$ parameter determines whether the resonance profile of the total cross section reaches its minimum on the low-energy side $(q>0)$ or the high-energy side $(q<0)$ of the resonance. This property will be referred to as the 'phase' of the resonance profile. Other shapes can also be obtained from these expressions, such as a window resonance $(q=0)$ or a noninterfering Lorentzian peak added to the background cross section $(|q| \gg 0)$.

The parametrization shown above is most applicable to the effect of an isolated resonance on the total photoabsorption cross section. In general, however, atomic Rydberg levels form a series whose members are not well-separated in energy and thus cainot be considered isolated. Equation (4a) is not easily adaptable to such a series of noninteracting, closely-spaced resonances because the background cross 
section appears as a multiplicative factor for each resonance, making a simple summation unsatisfactory. Shore ${ }^{34}$ has derived an equivalent expression that is better suited for a series of closely-spaced resonances because it is mathematically simpler to work with. His expression is

$$
\sigma_{t}=c+\sum_{k} \frac{B_{k}+A_{k} \varepsilon_{k}}{1+\varepsilon_{k}^{2}},
$$

where $C$ is the background cross section for the series of resonances, the summation is over the $k$ resonances, $\varepsilon_{k}$ is as defined in Eq. (4b) for each resonance, and $A_{k}$ and $B_{k}$ are the shape parameters for the kth resonance, which we shall refer to as the "Shore parameters". The value $C$ is understood to be a slowly-varying function of the photon energy. As with the Fano parameters, $q$ and $\rho^{2}$, the Shore parameters, $A$ and $B$, are assumed constant in the resonance region.

It is clear that for the case of a single, isolated resonance, the Fano and Shore parametrizations can be expressed in the same mathematical form, as follows:

$$
a_{t}=\sigma_{0}\left[\frac{c_{1}+c_{2} c+c^{2}}{1+c^{2}}\right] \text {, }
$$

where $C_{1}$ and $C_{2}$ can be expressed in terms of either $q$ and $\rho^{2}$ or $A$ and $B$. We note that although the shore formalism is to be preferred for multiple resonances, the parameters $A$ and $B$ are not dimensionless 
quantities as are $q$ and $p^{2}$. Because of this, the Fano formalism is more descriptive in the isolated-resonance case.

The formalisms presented so far were derived for the total cross section. In a photoemission experiment, however, partial cross sections are commonly measurjd. Starace ${ }^{35}$ has addressed the problem of several outgoing channels in the vicinity of an autoionization resonance. Davis and Feldkamp ${ }^{36}$ and Combet Farnoux ${ }^{37}$ have derived equivalent expressions. He shall use the notation of Starace. His expression for the partial cross section of each of the observable photoemission channels, $\mu$, is

$$
\begin{aligned}
& \sigma(u)=\frac{\sigma_{0}(u)}{1+\varepsilon^{2}}\left[\varepsilon^{2}+2\left\{q \operatorname{Re}\left(a_{\mu}\right)-\operatorname{Im}\left(a_{\mu}\right)\right\} \varepsilon+1\right. \\
& \left.-2 q \operatorname{Im}\left(a_{\mu}\right)-2 \operatorname{Re}\left(\alpha_{\mu}\right)+\left(q^{2}+1\right)\left|\alpha_{\mu}\right|^{2}\right] \text {, }
\end{aligned}
$$

where $\sigma_{0}(\mu)$ is the off-resonance partial cross section for the $\mu$ th observable final state and $c$ and $q$ are defined in Eqs. (4b) and (5), respectively. The complex parameter, $a_{\mu}$, is given $b y^{38}$

$$
a_{\mu}=\frac{\left\langle\phi|V|_{\mu}\right\rangle}{\langle g|\vec{r}| \mu\rangle}\left[\frac{2 \pi}{\Gamma} \sum_{\mu}\left(g|\vec{r}|_{\mu} x_{\mu}|V| \phi\right\rangle\right] \text {, }
$$

with $T$ given by Eq. (7). The term in brackets is common to all channels. The $a_{\mu}$ parameters can be thought of as replacing $p^{2}$ as the correlation coefficient for each channel when partial cross sections are medsured. It is important to noie that each $\mu$ represents 
an observable photoionization chanrel [eg. $\left.\mathrm{He}^{+}\left(1 \operatorname{se} \mathrm{p}_{1 / 2}\right)\right]$. This restriction was not necessary in the Fano and Shore derivations of the resonance behavior of the total cross section, because the individual photoemission channels only appeared in summations over $\mu$. It is clear that Eq. (11) has the same form as Eq. (10), because it describes the characteristic behavior of a cross section in the vicinity of an autoionization resonance. We will refer to $C_{1}(\mu)$ and $C_{2}(\mu)$ as the "Starace parameters".

Because all of the preceding formalisms, whether for total or particl cross sections, have the same mathematical form, it is possible to equate the parameters of the various formalisms, keeping in mind that expression of the parameters in terms of the appropriate matrix elements is only possible if the proper formalism for iiiy given experiment is used. For example, although effective Fano prameters can be derived for the autoionization profile of a partial cross section, it may be misleading to report them as the appropriate resonance parameters, because the expressions given by $F$ atio for $q$ and $0^{2}$ are not directly applicable to a partial cross section. They can be used in a descriptive context, however. This point will be discussed further in the next subsection.

An additional complication, discussed in Ref. 39, occurs because every peak, $m$, in a photoemission spectrum contains more than one of the channels, $\mu$, such as the $\mathrm{He}^{+}(1 \mathrm{~s})$ peak, which has contributions from two outgoing channels; $1 \operatorname{scp}_{1 / 2}$ and $1 \mathrm{sep}_{3 / 2} .^{40}$ Thus, the partial cross section for each photoelectron peak, $\sigma(m)$, is the sum of 
severa' $\sigma(\mu)$. The expresston for $\sigma(m)$ is of the same form as Eq. (11), but with $\sigma_{0}(\mu)$ replaced by the off-resonance partial cross section for the unresolved channels, ${ }_{0}(m)$, and $\operatorname{Re}\left(a_{\mu}\right), \operatorname{Im}\left(\alpha_{\mu}\right)$, and $\left|\alpha_{\mu}\right|^{2}$ replaced by Re $\langle a\rangle_{m} \cdot \operatorname{Im}\langle a\rangle_{m}$, and $\left\langle|\alpha|^{2}\right\rangle_{m}$, which are averaged quantities weighted by the $\sigma_{0}(\mu)$. The Schwartz inequality requires that

$$
(\operatorname{Re}(\omega) m)^{2}+\left(I m\langle\alpha\rangle_{m}\right)^{2} \leq\left\langle|\alpha|^{2}\right\rangle_{m},
$$

so that the modified Eq. (II) contains three unknowa quantities. Because a fit to the form of Eq. (10) only provides two parameters, it is, in general, impossible to solve for all three unknowns.

The angular-distribution asymmetry parameter, 8, can also show effects of autoionization. Kabachnik and Sazhina ${ }^{41}$ have shown that for photoionization in the region of an isolated resonance, $\beta$ is given by

$$
=\frac{X^{\prime} c^{2}+Y^{\prime} c^{\prime}+Z^{\prime}}{A^{\prime} c^{2}+B^{\prime} c+C^{\prime}},
$$

where $A^{\prime}, B^{\prime}$ and $C^{\prime}$ are defined in terms of the parameters for the cross section over the resonance, and $X^{\prime}, Y^{\prime}$ and $Z^{\prime}$ are new parameters that depend on the same matrix elements presented earlier, as well as their relative phases. Equation (14) was obtained by the division of two functions of the form of Eq. (10).

The parameters in Eq. (14) have been expressed ${ }^{41}$ in terms of the same dipole and Coulomb matrix elements usea in the description of 
the resonance behavior of total and partial cross sections. The expressions derived by Kabachnik and Sazhina describe the resonance effect upon the asymmetry parameter, $\beta_{t}$, for the total photoelectron flux from a given sample. Except possibily for the special case of no interchannel coupling in the continuum, these expressions cannot describe the resonance behavior of the asymmetry parameter, $\beta_{\mu}$, for an individual photoemission channel in terms of these matrix elements. This is especially true for helium because, as we have seen from the discussion in Sect. B, continuum interactions are important. While the form of Eq. (14) correctly describes these "partial B's", no detailed interpretation of the resulting parameters is yet possible.

\section{DATA ANALYSIS}

In the present experimeni, the resonance behavior of the $\mathrm{He}^{+}(n=2)$ partial cross section, branching ratio, and asymetry parameter in the region below the $n=3$ threshold was measured. The data for the $n=2$ satellite are shown in Figs. 6-8. The cross-section data were scaled to the data of Marr and West ${ }^{1}$ at the off-resonance energy of $68.9 \mathrm{eV}$. The remainder of this section is devoted to a discussion of the fitting techniques and assumptions used to describe analytically the cross-section and asymmetry-parameter data in the resonance region.

The $o_{n=2}$ data, shown in Fig. 6 , were fitted to the Shore formula, Eq. (9), convoluted with a truncated triangular function of 
full-width equal to $0.17 \mathrm{eV}(0.43 \mathrm{~A})$ to account for monochromator broadening. The off-resonance cross section, $C$, was taken to be a linear function of energy. The positions, $E_{0}$, and widths, $r$, of the four resonances were obtained from Woodruff and Samson. ${ }^{10}$ The Shore parameters derived in this way are presented in Table $I$. Note that the values for the fourth resonance were held fixed. The solid curve in Fig. 6 shows this fit, whereas the dashed curve is the same fit, but with the monochromator broadening removed.

The validity of using the Slore parametrization is dependent upon the assumption that the resonarices are not coupled in any way, or equivalently, that the series of discrete levels, $i_{i}$, do not perturb each other via Coulomb interactions. Shore ${ }^{34}$ has indicated that this is a good approximation provided that the radiative widths of the resonance states are small compared to their overall widths. Typical radiative lifetimes for allowed dipole transitions are in the range $10^{-8}-10^{-9} \mathrm{sec}^{42}$ so the radiative widths are several orders of magnitude smaller than the resonance widths.

The equivalent Starace parameters for $\sigma_{n=2}$ are also presented in Table $\$ because they are the most interpretable parameters for a partial cross section. From the approximation in the previous paragraph, we know that the Shore parameters derived from the fit to the $o_{n=2}$ data represent isolated-resonan':e parameters and can be equated to the Starace parameters. Use of Eq. (9) in the present analysis is simply a mathematical construct to derive the appropriate Starace parameters. 
The $n=2$ satellite branching ratio relative to the 1 s main line (Fig. 7) also shows strong resonance effects, mainly due to the changes in $\sigma_{n=2}$. The branching-ratio data were fitted to a ratio of the two cross sections, $\sigma_{n=2}$ and $\sigma_{1 s}$, each written as in Eq. (9). The parameters for the function in the numerator were taken directly from the fit to $\sigma_{n=2}$. For the denominator, $\sigma_{1 s}$, the background cross section was taken as a polynomial of first order in energy, and the resonance parameters were varied to get the best fit. The numerator and denominator were convoluted separately with the same monochromator bandpass function used for the fit to the $\sigma_{n=2}$ data. The parameters for $\sigma_{1 s}$ for the $3 \mathrm{~s} 3 \mathrm{p}$ resonance from this fit can be found in Table II. The uncertainties in the $\sigma_{1 s}$ parameters for the higher-lying resonances derived from the branching-ratio fit are too large for these parameters to be reported with any confidence.

The asymetry parameter for the $n=2$ satellite is strongly affected by autoionization, because the peak includes contributions from two satellites with very different off-resonance asymmetry parameters. Our results are shown in Fig. 8. We also have measured $\beta_{1 s}$ in the resonance region and found it to be $2.00(5)$. The $B_{n=2}$ data were fitted in a manner identical to the fit to the branching-ratio data, except the parameters in the denominator were taken from the fit to $\sigma_{n=2}$ (Table I). The function used was 


$$
B_{n=2}=\frac{\sum_{k} \frac{Y_{k}+X_{k} \varepsilon_{k}}{1+\varepsilon_{k}^{2}}+Z}{\sum_{k} \frac{B_{k}+A_{k} \varepsilon_{k}}{1+\varepsilon_{k}^{2}}+C} .
$$

The background value, $Z$, was assumed to be a second-order polynomial in energy. The resonance parameters, $X_{k}$ and $Y_{k}$, are presented in Table III, where they have been used to determine $X_{k}{ }^{\prime}, Y_{k}{ }^{\prime}$ and $Z_{k}{ }^{\prime}$ as in Eq. (14). As with the $n=2$ partial cross-section fit, we have equated the parameters in the fit to the numerator of $\beta_{n=2}$ with the corresponding single-resonance parameters described by Kabachnik and Sazhina. ${ }^{41}$ The parameters $A_{k}{ }^{\prime}, B_{k}{ }^{\prime}$ and $C_{k}{ }^{\prime}$ are not shown, but can easily be derived from the values in Table $I$ and Eqs. (14) and (15). We present these "Kabachnik-Sazhina parameters" for " $n=2$ " with the caveat that the definitions given in Ref. 41 do not allow easy interpretation for an individual photoemission line.

The fit to the $\beta_{n=2}$ data, including monochromator broadening, is shown in Fig. 8. The problem of deconvolution of instrumental broadening from the measured asymetry parameters is not straightforward, especially when the monochromator bandpass is an the order of, or larger than, the resonance linewidth. The method used here is the same as that described for the fit to $R_{21}$, but using Eq. (15). This method was chosen because the form of Eq. (15) is more amenable to fitting a series of closely-spaced resonances, and because the measured asymetry parameters are derived from the ratio of peak intensities in two analyzers. The deconvoluted curve is shown in Fig. 8. 


\section{RESULTS - CROSS SECTIONS AMD BRANCHING RATIOS}

The parameters for $\sigma_{n=2}$ for the first four members of the Rydberg series leading to the $n=3$ threshold are listed in Table $I$, along with the results of Hoodruff and Samson, 10 which agree with our results except for the $3 \mathrm{~s} 3 \mathrm{p}$ resonance. We attribute this small difference to the fact that the fit by Woodruff and Samson does not agree with their data for the first resonance as well as the fit presented here. The parameters for each member of the Rydberg series are fairly similar, as originally predicted by $F$ ano and Cooper. ${ }^{21}$ Results of several calculations $22,32,43-48$ of these positions and widths are sumarized in Ref. 10. Differences in the background cross sections ( $C$ and $\sigma_{0}$ in Table $I$ ) are due to differences in the scaling of the present data and the data in Ref. 10.

From the satellite branching ratio in Fig. 7, we have determined rough values of the $o_{1 s}$ resonance parameters. Qualitatively, we find that $a_{1 s}$ has the same phase as $\sigma_{n=2}$ for the 3s3p resonance. Our qualitative, as well as quantitative (Table II), results are in complete agreement $w i$ th recently published data on ${ }^{0} 1 s{ }^{12}$ Similarly, because $\sigma_{t}$ is just the sum of $\sigma_{1 s}$ and $\sigma_{n=2}$, the total cross section also must have the same phase for this resonance. Quantitatively, the situation for $\sigma_{t}$ is summarized in Table IV. This qualitative interpretation is in conflict with the phase of $\sigma_{1 s}$ over the $3 \mathrm{~s} 3 \mathrm{p}$ resonance that is required by the shape of $\sigma_{t}$ measured by Ohez and Ederer ${ }^{20}$ combined with the shape of $\sigma_{n=2}$ from the 
present results (the latter being in agreement with the fluorescence measurements of Woodruff and Samson ${ }^{10}$ over the same region). The results from Dhez and Ederer :iould require $\sigma_{1 s}$ and $\sigma_{n=2}$ to have opposite phases for this resonance because the effect on $\sigma_{t}$ measured by them is small enough that when the two partial cross sections are added to get the total cross section, their effects would have to cancel. This requires that $\sigma_{1 s}$ reach a maximum below the resonance energy and a minimum above that energy. This conciusion was first reached by Woodruff and Samson, ${ }^{10}$ but is inconsistent with our results, as well as the direct measurement of $\sigma_{1 s^{\circ}}{ }^{12}$ Because this cancellation does not occur, the strength of the effect on $\sigma_{t}$ (as measured by $\rho^{2}$ ) must be significantly larger than that reported by Dhez and Ederer. This discrepancy may be explained by reference to Table I in Ref. 20. This table lists the Shore parameters for a series of five transmission scans taken at three different pressures; 50,90 and 120 torr. Calculating $q$ and $p^{2}$ for each scan shows $a$ definite pressure dependence of $\rho^{2}(0.009$ at 120 torr, 0.013 at 90 torr, and 0.018 at 50 torr). Exactly what can cause such a pressure effect on $\rho^{2}$ is unclear.

Our value of $q$ also disagrees with the previous result. While this may be due to our experimental uncertainties, it also could be the result of the normalization procedure used in the earlier work. By normalizing the data at $177.22 \AA$, which is an energy near the center of the resonance, that point is forced to lie on the background curve, fixing the shape of the resonance with respect to the background cross 
section and thus affecting $q$.

The present results are also to be compared with previous estimates of the resonance parameters. Fano and Cooper ${ }^{21}$ estimated $q$ and $\rho^{2}$ to be 1.7 and 0.01 , respectively. Calculations by Senashenko and Wague ${ }^{22}$ using the diagonalization approximation yielded $q=1.31$ and $\rho^{2}=0.019$. Both of these calculations disagree with the larger value of $\rho^{2}$ inferred here. For the first estimate, however, it may be possible to trace one reason for this disagreement. In estimating $q$, Fano and Cooper assumed that the matrix elements involving the 1 sep continuum state for the $3 \mathrm{~s} 3 \mathrm{p}$ resonance are not significantly different than similar matrix elements for the 2s2p resonance. This assumption seems to imply, at least, that $\sigma_{15}$ has the same phase for both the $2 s 2 p$ and the $3 s 3 p$ resonances. However, ${ }^{0}$ Is has opposite phases for these two resonances. The effect on the resulting values of $q$ and $p^{2}$ for the total cross section is not clear. One other calculation ${ }^{43}$ of $o_{t}$ for the $3 \mathrm{~s} 3 \mathrm{p}$ resonance has also been performed that reproduces the correct qualitative shape of the resonance, but no parameters were extracted because of the small number of points evaluated.

The remainder of this subsection will illustrate some of the quantitative results that can be derived from the measured parameters (Table I) and the parameters inferred for $\sigma_{1 s}$ and $\sigma_{t}$ (Tables II and IV). We wish to stress that caution should be exercised concerning the actual values presented below, but the procedures described are useful in general for autoionization phenomena. 
The oscillator strength, $f$, for the $353 p$ resonance can be odtained from the Fano parameters for the total cross section. It is given by ${ }^{49}$

$$
f=\left(0.195 R y^{-1} M b^{-1}\right) q^{2} \rho^{2} \sigma_{t} \Gamma,
$$

with $\Gamma$ expressed in Rydbergs and $o_{t}$ expressed in Mb. Using the values in Table IV, we find $f=2.0 \times 10^{-4}$, which agrees well with the previous estimate 21 of $1.2 \times 10^{-4}$. A similar, but possibly more descriptive, expression for $f$ is obtained ${ }^{50}$ by replacing $q^{2}$ in Eq. (16) with $\left(q^{2}-1\right)$. The result for this case is $-8.1 \times$ $10^{-5}$, indicating that $a_{t}$ in the vicinity of the $3 \mathrm{~s} 3 \mathrm{p}$ resonance exhibits a net loss in oscillator strength compared to the background cross section. The latter value of $f$ is more descriptive of autoionization in the sense that it is a measure of the spectral repulsion part of the autoionization profile.

Useful information also may be derived from the partial cross sectons. Although, as we have pointed out, it is generally impossible to determine all three unknowns in Eq. (13), the simplicity of the helium system allows us to do so in a manner similar to the method described in an earlier paper. ${ }^{39}$ The following discussion is 1 imited to the $3 \mathrm{~s} 3 \mathrm{p}$ resonance, but sinsilar results are expected for the nigherlying resonances. For $\mathrm{He}^{+}(1 \mathrm{~s})$ production, there are only two outgoing channels, $\mu: 1 \operatorname{sep}_{1 / 2}$ and $1 s \in p_{3 / 2} \cdot 40$ The dipole and coulomb matrix elements for these two channels help determine two of the $a_{\mu}$ parameters: $\alpha_{1 s c p_{1 / 2}}$ and $a_{1 s c p_{3 / 2}}$ of course, both of these channels 
are present in the $\mathrm{He}^{+}(1 \mathrm{~s})$ peak $(\mathrm{m}=1 \mathrm{~s})$. In this case, however, because the spin-orbit interaction in the ep continuum is small, ard because $\beta_{1 s}$ is identically 2.0 over the resonance, the Scinartz inequality, Eq. (13), becomes an equality. Equations (10), (11) and (13) and the Starace parameters in Table II may then be used to obtain Re(a) $1 s, I m\langle a\rangle_{1 s}$ and $\left(|a|^{2}\right\rangle_{1 s}$. The solution involves a complicated quadratic equation for $\operatorname{Re}(\alpha)_{1 s^{*}}$. The solution with $\left(|\alpha|^{2}\right)_{1 s}>2$ is dismissed, because it would require that the total cross section have $\rho^{2}>1$. The parameters for the correct solution are given in Table $v$. It is also possible to determine the non-averaged quantities, $\operatorname{Re}\left(\alpha_{1 \operatorname{se} p_{j}}\right), \operatorname{Im}\left(\alpha_{1 \operatorname{sep}}\right)$ and $\left|\alpha_{1 \operatorname{sep}}\right|_{j}^{2}$, where $j$ can have the values $1 / 2$ or $3 / 2$, because our earlier assumption that Eq. (13) is an equality means that the matrix elements for the two outgoing channels in the is peak are identical for the dipole and Coulomb interactions. The averaged quantities for the $1 \mathrm{sep}$ final state in Table $V$ are then just averages of identical quantities.

From Eqs. (15) and (16) in Ref. 39, we can determine the partial linewidths, $\Gamma_{1 s}$ and $\Gamma_{n=2}$, for the two final states. Both of these partial widths are included in Table $V$. The large difference in the partial widths illustrates why the effect of the resonance on $\sigma_{1 s}$ is small relative to the effect on $\sigma_{n=2}$, even though $\sigma_{0}(1 s)$ is an order of magnitude larger than $\sigma_{0}(n=2)$. The partial widths can be interpreted as an additional measure $\left(\left\langle|a|^{2}\right)_{m}\right.$ is the other one) of the strength of the resonance effect on an individual final state. As we did with the a parameters for the 15 level, we can break down the 
contributions to $\Gamma_{1 s}$ into partial widths for each outgoing channel, being careful to account for the multiplicities of the two outgoing channels. The results are $\Gamma_{1 \operatorname{sep}_{1 / 2}} / \Gamma=0.004$ and $\Gamma_{1 \operatorname{sep}_{3 / 2}} / \Gamma=0.009$. The partial widths (in percent) derived here agree very well with previous calculations. 22,32

Because we have already found $\left\langle|a|^{2}\right\}_{15}$, we can use Eq. (16) in Ref. 38 to find $\left\langle|\dot{\alpha}|^{2}\right\rangle_{n=2}$. Having done this, $c_{1}(n=2)$ and $c_{2}(n=2)$ for the $353 p^{-}$resonance from Table I can be used with Eqs.

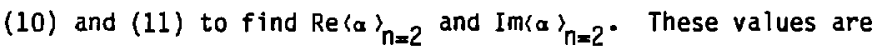
also presented in Table $V$. We note that for the a-parameters for $\sigma_{n=2}$, Eq. (13) appears to be a true inequality. Because the satellite peak containing the $2 s$ and $2 p$ final states includes seven possible outgoing channels, no further information can be obtained. A check of the $a_{\mu}$ parameters can be made as described in Ref. 39. The results in Table $V$ satisfy this check to well within the statisticai errors, suggesting that no major systematic errors are present in the data analysis.

Some interpretation of the $\alpha_{\mu}$ parameters can be made, keeping in mind that the actual values may not be very accurate. The positive

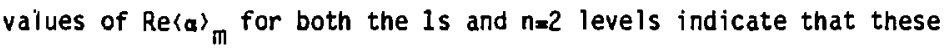
two resonance profiles should have the same phase over the resonance, as we have suggested. The approximately zero values for $I_{m}(a)$ is and $I m\{a\rangle_{n=2}$ show that the $a_{\mu}$ parameters are essentially real numbers. This result seems fortuitous, because it probably does not imply, as discussed by Combet Farnoux, ${ }^{37}$ that interchannel coupling in the 
continuum is weak. In fact, because the $n=2$ peak is a satellite of the 1s peak, interchannel coupling in this case is important (see Sect. B). It might prove interesting to measure the a parameters for the individual final states, $2 s$ and $2 p$. The strong coupling between $2 \mathrm{sep}$ and $2 \mathrm{pes}$ may result in a parameters that are complex. A detailed flourescence experiment, similar to that done by Woodruff and S.alson, ${ }^{10}$ could measure these parameters.

To this point, we have only derived parameters which depend on several of the dipole and Coulomb matrix elements. It is possible, however, for the case of the $3 s 3 p$ resonance, to determine directly the squares of three of the matrix elements, including all of those describing autoionization into the lsep continuum. The Coulomb matrix elements $\left|\left\langle 3 s 3 p|V| 1 s \varepsilon p_{j}\right\rangle\right|^{2}$, with $j=1 / 2$ or $3 / 2$, can be obtained from Eq. (7) for the partial decay width, $\Gamma_{1 \mathrm{~s}}=0.0023 \mathrm{eV}$, by properly accounting for the multiplicity of the $1 s \in p_{j}$ states. The dipole matrix elements for continuum absorption, $\left|\left\langle 1 s \in p_{j}|\vec{r}| l s^{2}\right\rangle\right|^{2}$, can be determined from $a_{1 s}$ as described in Ref. 21 and again considering the multiplicities. The dipole matrix elements can also be expressed as oscillator strengths. The dipole matrix element for the discrete transition $1 s^{2} \rightarrow 3 s 3 p$ can be determined ${ }^{21}$ from the oscillator strength, $f$, derived above. All of these results are listed in Table VI, aiong with estimates of two of the matrix elements by Fano and Cooper. ${ }^{21}$ Their results agree with ours to within a factor of 2 . One additional parameter can also be derived. The square of the term in brackets in Eq. (13) can be determined by using $\left\langle|a|^{2}\right\rangle_{1 \mathrm{~s}}$ from 
Table $V$ and the matrix elenents in Table VI. We find this term to have the value $36 \mathrm{Mb} / \mathrm{Ry}^{2}$.

A review of the results of this subsection is useful, Quantitatively, we have presented parameters defining the resonance profiles of $\sigma_{n=2}$ and $R_{21}$. From these results, we have inferred the qualitative behavior of $\sigma_{1 s}$ and $\sigma_{t}$ for the $3 \mathrm{~s} 3 \mathrm{p}$ resonance only, concluding that the phases for $\sigma_{n=2}, \sigma_{1 s}$, and $\sigma_{t}$ are the same for this resonance. This result agrees with recent photoemission data, but disagrees with an earlier photoabsorption measurement. Exercising caution for the quantitative results, we have determined paraneters that quantify the qualitative behavior of $\sigma_{1 s}$ and $\sigma_{t}$, in order to illustrate methods for extracting information from measurements of autoionization phenomena. The results, such as a parameters and dipole matrix elements, which are obtained in this way can be useful in describing the aspects of interchannel coupling in the continuum 37 and for comparison with theoretical calculations of resonance behavior. Values for individual matrix elements may prove particularly helpful as a guide to determining appropriate wavefunctions and other parameters for calculations. As a final point, we again wish to stress that while the results of this experiment are not sufficiently accurate to provide a complete and quantitative interpretation, we have attempted to document fully one of the first measurements of this kind. 


\section{RESULTS - ASYMMETRY PARAMETERS}

The data in Fig. 8 mark the first detailed measurement of a satellite asymmetry parameter over autoionization resonances. The accolupanying parameters in Table III vary only slightly over the members of the Rydberg series, as first predicted by $0 i 11.51$

Because the angular distributions of all of the helium photoemission peaks (there are only 2) were measured in this experiment, it is possible to determine $B_{t}$ from

$$
B_{t}=\frac{{ }_{15}+R_{21}{ }^{\beta} n=2}{1+R_{21}},
$$

with $\beta_{1 s}=2$, and $R_{21}, \sigma_{n=2}$ and $\beta_{n=2}$ given by Eqs. (10) and (15) for the $3 \mathrm{~s} 3 \mathrm{p}$ resonance only. The resulting $\beta_{t}$ has the same mathematical form as $B_{n=2}$, and the Kabachnik-Sazhina parameters describing it are given in Table VIi. The off-resonance value of $\beta_{t}$ is approximately 1.8, with deviations due to autoionization of on $1 y \sim 0.1$, as expected because the dominant is channel shows no effect in $\beta_{15^{\circ}}$. It is hoped that these results, as well as those for $\beta_{n=2}$, will spur furtiner theoretical development regarding the detailed behavior of angular distributions of individual photoemission lines in the vicinity of autoionization resonances. Complete understanding of this phenomenon awaits further theoreticai and experimental work.

In Sect. $B$ we were able to derive the ratio $R=\sigma_{2 p} / \sigma_{2 s}$ from the measured off-resonance $\beta_{n=2}$ data and calculated values of $\beta_{2 p}$. 
We are unable to do this in the resonance region because the resonance behavior of $\beta_{2 p}$ is unknown, but certain qualitative statements can be made concerning the effects on $R$ of the $3 s 3 p$ resonance. For the following discussion, the reader is referred to the deconvoluted curves in Figs. 6 and 8.

Figure 6 shows that the $n \times 2$ partial cross section drops nearly to zero at the minimum of the $3 \mathrm{~s} 3 \mathrm{p}$ resonance. From Fig. 5 , the background value of $R$ at $70 \mathrm{eV}$ is -2.2 . To account for the minimum in $\sigma_{n=2}$, both $\sigma_{2 p}$ and $\sigma_{2 s}$ must be going through a minimum at the energy of the minimum in $a_{n=2}$. Furthermore, both of these minima occur on the low-energy side of the $3 \mathrm{~s} 3 \mathrm{p}$ resonance. In other woros, we can conclude that both $\sigma_{2 p}$ and $\sigma_{2 s}$ are affected by the resonance and that they have the same phase. The question then arises of whether or not these effects manifest themselves in an effect on $R$. If $R$ is left unaffected, then the measured change i $B_{n=2}[$ see Eq. (2)] must be due solely to changes in $B_{2 p}$ (assuming that $B_{2 s}$ is always 2.0 , just like $\left.\beta_{1 s}\right)$. However, examination of the minimum in $\beta_{n=2}$ on the high-energy side of the resonance shiwws that even if $B_{2 p}$ is -1 at this energy, a valuse of $R=2.2$ is not large enough to yield [see Eq. (3)] the deconvoluted value of $\beta_{n=2}=-0.25$ from $F i g .8$. The values of $R=2.2$ and $B_{2 p}=-1$ yield $B_{n=2}=-0.06$. The uncertainty in the minimum of the deconvoluted curve for $\beta_{n=2}$ is $\sim 0.1$. Therefore, our value of $s_{n=2}=-0.25$ suggests that $R$ shows a positive deviation from its background value of 2.2 on the high-energy side of the $3 \mathrm{~s} 3 \mathrm{p}$ resonance. Because $R$ must have the same shape as the satellite 
branching-ratio over an autoionization resonance, it must reach a minimum on the low-energy side of the $3 \mathrm{~s} 3 \mathrm{p}$ resonance as wel1. No limits can be placed on the value of this minimum. The conclusion then, is that $R$ drops to a minimum on the low-energy side and rises to a maximum on the high-energy side of the $3 \mathrm{~s} 3 \mathrm{p}$ resonance. This behavior is most likely caused by $\sigma_{2 p}$ and $\sigma_{2 s}$ having similar profiles, but with $\sigma_{2 p}$ reaching its minimum at a slightly lower energy than $\sigma_{2 s}$. This implies that the Starace parameters, $c_{1}(2 p)$ and $C_{2}(2 p)$, are slightly larger than $C_{1}(2 s)$ and $C_{2}(2 s)$, respectively. He expect the higher members of this series to have similar effects on $R$, because partial cross sections tend to retain the same shape over a Rydberg series. 21

\section{CONCLUSIONS}

The photoionization of helium to the $n=2$ excited state of the helium ion has provided several interesting results. The off-resonance measurements of ${ }_{n=2}$ have shown additional clear evidence that the $n=2$ satellite is mainly comprised of the $2 p$ final state near threshold. Furthermore, the strong energy dependence of $R$ has given some insight into the understanding of electron correlation in atomic systems: the helium case being especialiy useful because of its relative simplicity.

For the $\mathrm{He}^{+}(\mathrm{n}=2)$ satellite, the partial cross-section, branching-ratio, and asymetry-parameter behavior has been measured over the major Rydberg series leading to the $n=3$ thrcshold. These 
angular-distribution measurements are the first of their kind for a satellite line. Parameters describing all of these resonance effects have been presented. From the angular-distribution results, the qualitative behavior of $R$ over the resonances has been inferred. The qualitative behavior of the total and is cross sections has been determined for the $3 s 3 p$ resonance. The results have mixed agreement with previous measurements, and more work at higher resolution on the total cross section of helium above the $n \geq 2$ thresholds is recommended to verify our conclusions. The 1s cross-section results have been used in an illustrative way to indicate how information about autoionization behavior in photoernission can be extracted. 
where $L_{t}$ is the length of the flight path of the TOF analyzers $(\sim 23$ $\mathrm{cm})$, and $\mathrm{C}$ is a constant. This method works at SSRL because the synchrotron radiation is pulsed as a result of the bunched structure of the electrons in the storage ring. The pulses are narrow $\{\sim 0.3$ nsec) and spaced far enough apart in time (195-780 nsec depending on the running mode of SPEAR) to allow electrons of most energies to be collected between consecutive pulses with moderate-to-good time resolution. The limitation on the TOF analyzer resolution at low electron kinetic energies is the uncertainty in the flight path (and hence the flight time) because of the finite width $(3 \mathrm{~mm})$ of the photon beam at the interaction region. For the grasshopper monochromator, the resolution was typically $2.5 \%$ of the kirietic energy of the electrons for the $0^{\circ}$ analyzer, and 3.5-4.0\% for the $54.7^{\circ}$ detector. At high kinetic energies ( $>100-200 \mathrm{eV})$, other contributions to the resolution become important, and the poor overall analyzer resolution is prohibitive to doing well-resolved experiments.

The TOF method is well-suited to gas-phase photoelectron spectroscopy because it collects nearly all energies of photoelectrons simultaneously, providing an excellent signal-to-noise ratio for many experiments. Simultaneous measurement of photoelectron peak intensities at two angles yields asymmetry parameters (see Eq. 4, Chapter I) that are independent of variations in the photon flux and gas pressure. Peak intensities at $54.7^{\circ}$ (the 'magic angle'), for which $P_{2}(\cos \theta)$ vanishes, are directly proportional to photoionization cross sections after normalization to photon flux and gas 
15. V.L. Jacobs and P.G. Burke, J. Phys. B $\underline{5}, L 67$ (1972).

16. T.N. Chang, J. Phys. B $\underline{13}, L 551$ (1980).

17. J.A. Richards, Honours Thesis, Monash University (1981).

18. K.A. Berrington, P.G. Burke, W.C. Fon, and K.T. Tayior, J. Phys. B 15, L603 (1982).

19. J.A. Richards and F.P. Larkins, J. Electron Spectrosc. 32, 193 (1983).

20. P. Dhez and D.L. Ederer, J. Phys. B 6 , L59 (1973).

21. U. Fano and J.W. Cooper, Phys. Rev. 137, A1364 (1965).

22. V.S. Senashenko and A. Wagué, J. Phys. B 12, L269 (1979).

23. S.T. Manson, J. Electron Spectrosc. 9, 21 (1976).

24. J.A. Richards and F.P. Larkins (private communication).

25. T. Ishihara, J. Mizuno, and T. Watanabe, Phys. Rev. A, 22, 1552 (1980).

26. H.P. Kelly, Phys. Rev. A $\underline{6}, 1048$ (1972).

27. R.L. Brown, Phys. Rev. A 1, 341 (1970).

28. J.M. Bizau, F.J. Wuilleumier, D.L. Ederer, P. Dhez, S. Krummacher, and V. Schmidt (unpublished).

29. C.H. Greene, Phys. Rev. Lett. 44, 869 (1980).

30. C.H. Greene (private communication).

31. R.P. Madden and K. Codling, Astrophys. J. 141, 364 (1965).

32. D.R. Herrick and 0. Sinanoğlu, Phys. Rev, A 11, 97 (1975).

33. U. Fano, Phys. Rev. 124, 1866 (1961).

34. B.W. Shore, Phys. Rev. 171, 43 (1968).

35. A.F. Starace, Phys. Rev. A 16, 231 (1977). 
36. L.C. Davis and L.A. Feldkamp, Phys. Rev. B 15, 2961 (1977); 23, $6239(1981)$.

37. F. Combet Farnoux, Phys. Rev. A $\underline{25}, 287$ (1982).

38. P.C. Kemeny, J.A.R. Samson, and A.F. Starace, J. Phys. B 10, L201 (1977).

39. P.H. Kobrin, U. Becker, S. Southworth, C.M. Truesdaie, D.W. Lindle, and D.A. Shirley, Phys. Rev. A 26, 842 (1982).

40. Although the $1 \mathrm{se} \mathrm{p}_{1 / 2}$ and $1 \mathrm{sep}_{3 / 2}$ final states are not resolved in this work, they have to be considered here for the later discussion because of their different multiplicities. Ignoring this point can lead to incorrect interpretation of the data.

41. N.M. Kabachnik and. I.P. Sazhina, J. Phys. B $\underline{9}, 1681$ (1976).

42. G. Herzberg, Molecular Spectra and Molecular Structure I. Spertra of Diatomic Molecules (Van Nostrand, New York, 1950).

43. S. Ormonde, W. Whitaker, and L. Lipsky, Phys. Rev. Lett. 19, 1161 (1967).

44. P.G. Burke and A.J. Taylor, J. Phys, $8 \underline{2}, 44$ (1969).

45. R.S. Oberoi, J. Phys. B $\underline{5}, 1120$ (1972).

46. K.T. Chung and I. Chen, Phys. Rev. Let.t. $\underline{28}, 783$ (1972).

47. Y.K. Ho, J. Phys. B $\underline{12}, 387$ (1979).

48. Y.K. Ho, J. Phys. B $15, L 691$ (1982).

49. U. Fano and J.W. Cooper, Rev. Mod. Phys. 40, 441 (1968).

50. P.G. Burke and D.D. McVicar, Proc. Phys. Soc. (London) 86, 989 (1965).

51. D. Dill, Phys. Rev. A $\underline{7}, 1976$ (1973). 
Table I. Parameters for the $\mathrm{He}^{+}(\mathrm{n}=2)$ partial cross section for the first 4 members of the $1_{n}$ Rydberg series. The background cross section, $o_{0}$ (Mb), was taken to be $0.216-0.0017(E)$, where $E$ is the photon energy in eV. Numbers in parentheses represent statistical errors only.

\begin{tabular}{|c|c|c|c|c|c|c|}
\hline \multirow[b]{2}{*}{ Resonance } & \multicolumn{3}{|c|}{ Shore parameters (Mb) } & & \multicolumn{2}{|c|}{ Starace parameters } \\
\hline & & This work & $W S^{a}$ & & This work & $W S^{a}$ \\
\hline $1_{3}$ & $A$ & $0.120(2)$ & $0.081(14)$ & $c_{1}$ & $0.55(2)$ & $0.24(11)$ \\
\hline$E_{0}=69.917 \mathrm{eV}^{\mathrm{a}}$ & B & $-0.044(2)$ & $-0.065(8)$ & $c_{2}$ & $1.24(2)$ & $0.94(18)$ \\
\hline$\Gamma=0.178 \mathrm{ev}^{\mathrm{a}}$ & $\mathrm{c}$ & $0.097(1)$ & $0.086(7)$ & $o_{0}(M b)$ & $0.097(1)$ & $0.086(7)$ \\
\hline $1_{4}$ & A & $0.086(5)$ & $0.079(17)$ & $c_{1}$ & $0.35(5)$ & $0.23(13)$ \\
\hline$E_{0}=71.601 \mathrm{ev}^{\mathrm{a}}$ & B & $-0.061(5)$ & $-0.066(10)$ & $c_{2}$ & $0.92(5)$ & $0.92(21)$ \\
\hline$\Gamma=0.096 \mathrm{ev}^{\mathrm{a}}$ & C & $0.094(1)$ & $0.086(7)$ & $\sigma_{0}(M b)$ & $0.094(1)$ & $0.086(7)$ \\
\hline $1_{5}$ & $A$ & $0.080(7)$ & $0.088(21)$ & $c_{1}$ & $0.45(8)$ & $0.49(15)$ \\
\hline$E_{0}=72.181 e v^{a}$ & $B$ & $-0.051(7)$ & $-0.044(12)$ & $c_{2}$ & $0.86(8)$ & $1.02(26)$ \\
\hline$\Gamma=0.067 \mathrm{ev}^{\mathrm{a}}$ & C & $0.093(1)$ & $0.086(7)$ & $\sigma_{0}(M b)$ & $0.093(1)$ & $0.086(7)$ \\
\hline $1_{6}$ & $A$ & $0.080(f i x)$ & $0.085(28)$ & $r_{1}$ & $0.45(f i x)$ & $0.23(20)$ \\
\hline$E_{0}=72.453 \mathrm{ev}^{\mathrm{a}}$ & $B$ & $-0.051(f i x)$ & $-0.066(16)$ & $\mathrm{C}_{2}$ & $0.86(f i x)$ & $0.99(34)$ \\
\hline$\Gamma=0.038 \mathrm{ev}^{\mathrm{a}}$ & $c$ & $0.093(1)$ & $0.086(7)$ & $\sigma_{0}(M b)$ & $0.093(1)$ & $0.086(7)$ \\
\hline
\end{tabular}

Woodruff and Samson, Ref, 10. 
$-109-$

Table II. Parameters for the $\mathrm{He}^{+}(1 \mathrm{~s})$ partial cross section for the $3 \mathrm{~s} 3 \mathrm{p}$ resonance. The background cross section, $\sigma_{0}(M b)$, was taken to be $2.87-0.0283(E)$, where $E$ is the photon energy in lV. Numbers in parentheses represent statistical errors only.

\begin{tabular}{cc}
\hline Effective Fan parameters ${ }^{a}$ & Starace parameters ${ }^{a}$ \\
\hline$q=1.1(3)$ & $C_{1}=1.01(3)$ \\
$\rho^{2}=0.046(30)$ & $C_{2}=0.10(7)$ \\
$\sigma_{0}=0.892(20) \mathrm{Mb}$ & $\sigma_{0}=0.892(20) \mathrm{Mb}$ \\
\hline $\mathrm{a}_{0}=69.917(12)$ aV and $\Gamma=0.178(12)$ el from Ref. 10.
\end{tabular}


Table III. Parameters for $\beta_{n=2}$ for the first 4 members of the $1_{n}$ Rydterg series. The background value, $Z$ (Mb), was taken to be $2.28+0.0103(E)-0.00061\left(E^{2}\right)$, where $E$ is the photon energy in $\mathrm{eV}$. Numbers in parentheses represent statistical errors on $7 y$.

\begin{tabular}{|c|c|c|c|c|c|c|}
\hline \multirow[b]{2}{*}{$n$} & \multicolumn{3}{|c|}{ Fit parameters (Mb) } & \multicolumn{3}{|c|}{ XS parameters $(M b)^{a}$} \\
\hline & $x$ & $Y$ & $z$ & $x^{\prime}$ & $Y^{\prime}$ & $Z^{\prime}$ \\
\hline
\end{tabular}

$$
\begin{array}{lllllll}
3 & -0.030(6) & -0.037(5) & 0.021(1) & 0.021(1)-0.030(6) & -0.016(5) \\
4 & -0.031(9) & -0.037(9) & 0.036(2) & 0.036(2)-0.031(9) & -0.001(9) \\
5 & -0.031(14) & -0.040(14) & 0.041(3) & 0.041(3)-0.031(14) & 0.001(14) \\
6 & -0.031(f i x) & -0.040(f i x) & 0.043(3) & 0.043(3) & -0.031(f i x) & 0.003(f i x)
\end{array}
$$

$a_{\text {Kabachnik and Sazhina, Ref. } 41 .}$ 
Table IV. Parameters for the total cross section of helium for the $3 s 3 p$ resonance, derived from the results in Tables $I$ and $I I$. The numbers in parentheses represent statistical errors only.

\begin{tabular}{|c|c|c|c|c|c|}
\hline & \multicolumn{2}{|c|}{ Fano parameters } & & \multicolumn{2}{|c|}{ Shore parameters (Mb) } \\
\hline & This work & $D E^{a}$ & & This work & $D E i$ \\
\hline$q$ & $0.84(30)$ & $1.36(20)$ & A & $0.18(8)$ & $0.032(6)$ \\
\hline $0^{2}$ & $0.11(3)$ & $0.012(3)$ & B & $-0.032(56)$ & $0.010(5)$ \\
\hline${ }_{0}(M b)$ & $0.989(20)$ & $0.957(30)$ & c & $0.989(20)$ & $0.957(30)$ \\
\hline$r(e v)$ & $0.178(12)^{b}$ & $0.132(14)$ & & & \\
\hline$E_{0}(E V)$ & $69.917(12)^{b}$ & $69.919(7)$ & & & \\
\hline
\end{tabular}


Table $V$. a-parameters and partial linewidths for the $3 s 3 p$ resonance.

\begin{tabular}{|c|c|c|c|c|}
\hline Final state & $\operatorname{Re}\langle a\rangle m$ & $\operatorname{Im}\langle\alpha\rangle m$ & $\left\langle|a|^{2}\right\rangle m$ & $\left(\Gamma_{m} / \Gamma\right) \times 100$ \\
\hline $1 \mathrm{sep}$ & $0.023(21)$ & $-0.03(4)$ & $0.0016 \begin{array}{l}+0.0020 \\
-0.0016\end{array}$ & $1.3+1.7$ \\
\hline $\left.\begin{array}{l}2 s \in p \\
2 p e s\end{array}\right\} n=2$ & $0.99(15)$ & $0.2(3)$ & $1.1(3)$ & $98.7^{+1.3}-1.7$ \\
\hline
\end{tabular}


Table VI. Matrix elements for the $3 s 3 p$ resonance.

\section{Amplitude}

Matrix element This work

Fano and Cooper2I

$\left|\left\langle 3 \mathrm{~s} 3 \mathrm{p}|\overrightarrow{\mathrm{r}}| 1 \mathrm{~s}^{2}\right\rangle\right|^{2}$

$1.1 \times 10^{-3} \mathrm{Mb}$

$6.8 \times 10^{-4} \mathrm{Mb}$

$\left|\left\langle 1 s \in p_{j}|\vec{r}| 1 s^{2}\right\rangle\right|^{2^{a, b}}$

$0.10 \mathrm{Mb} / \mathrm{Ry}$

$\left|\left\langle 3 s 3 p|V| 1 \operatorname{sep}_{j}\right\rangle\right|^{2^{b}}$

$4.5 \times 10^{-6} R y$

$4.7 \times 10^{-6} \mathrm{Ry}^{\mathrm{C}}$

The corresponding oscillator strength, $\mathrm{df} / \mathrm{dE}$, is $0.0014 \mathrm{eV}^{-1}$.

$b_{j}=1 / 2,3 / 2$.

"The value given by $F$ ano and Cooper is for the sum over all the channels contributing to the $1 \mathrm{~s}$ peak. We have divided their value by 6 for comparison. 


$$
-114-
$$

Table VII. Kabachnik-Sazhina parameters for the angular distribution of the total photoelectron flux from helium for the $353 p$ resonance. The numbers in parentheses represent statistical errors only.

$$
\begin{array}{ll}
X^{\prime}=1.81(4) & A^{\prime}=0.99(2) \\
Y^{\prime}=0.15(13) & B^{\prime}=0.18(8) \\
Z^{\prime}=1.79(7) & C^{\prime}=0.96(6)
\end{array}
$$




\section{FIGURE CAPTIONS}

Fig. 1. Energy-level diagram for helium. $D I$ = direct ionization. AI - autoionization. The energy scale above the break is expanced four times relative to the energy scale below the break.

Fig. 2. TOF photoelectron spectrum of helium taken with the $54.7^{\bullet}$ detector at a photon energy of $80 \mathrm{ev}$. The peak labels indicate the principal quantum number of the single-electron final state of the ion.

Fig. 3. Branching ratio, $R_{21}=\sigma_{n=2} / \sigma_{1 s}$, for the $\mathrm{He}^{+}(n=2)$ satelite relative to the $1 \mathrm{~s}$ main line. Experimental results; solid circles - present results; solid square - Samson, Ref. 4; open circle - Krause and Wuilleumier, Ref. 6; $X$ - Wuilleumier et al., Ref. 8. Theoretical curves; long-short dashed Salpeter and Zaidi, Ref. 13; dashed-dotted - Jacobs, Ref. 14; solid - Jacobs and Burke, Ref. 15; long-dashed - Chang, Ref. 16; short-dashed - Richards, Ref. 17. Where applicable (Refs. 14-17), we have plotted only the velocity results for consistency.

Fig. 4. Asymmetry parameter of the $\mathrm{He}^{+}(\mathrm{n}=2)$ satellite as a function of photon energy. Experimental results; solid circles present results; open circles - Bizau et a1, Ref. 9; $x$ Schmidt et al., Ref. 11; open squares - Morin et al., Ref。 12. Some error bars have been omitted for clarity. Theoretical curves; solid - Jacobs and Burke, Ref. 15; dashed Chang from Bizau et al., Ref. 9. Also shown are calculations 
of the asymmetry parameter for the $2 p$ final state $b y$ ijacobs and Burke (Ref. 15) and Chang (Ref. 9). The velocity forms of the calculations have been plotted in all cases.

Fig. 5. The subshell branching ratio, $R=\sigma_{2 p} / \sigma_{2 s}$, for the $\mathrm{He}^{+}(n=2)$ satellite as a function of photon energy. Exrerimental results; solid circles - present results; solid squares Woodruff and Samson, Ref. 10; open circles - Bizau et al., Ref. 9; X - Schmidt et a1., Ref. 11; open squares - Morin et al., Ref. 12. Some error bars have been omitted for clarity. Theoretical curyes; dashed-dotted - Jacobs, Ref. 14; solid Jacobs and Burke, Ref. 15; long-dashed - Chang, Ref. 16; short-dashed - Richards and Larkins, Ref. 19; long-short dashed - Berrington et al., Ref. 18. From Berringtor et ai. we show the length form of their calculation, which the authors predict to be more accurate than the velacity form. The remainder of the curves are velocity forms.

Fig. 6. Partial cross section of the $\mathrm{He}^{+}(\mathrm{n}=2)$ satellite in the resonance region below the $n=3$ threshold, scaled to Marr and West (Ref. 1) at $68.9 \mathrm{eV}$. The solid curve is a fit to the data using the form of Eq. (9). The dashed curve is the same fit with the monochromator brondening of $0.17 \mathrm{eV}(0.43 \mathrm{~A})$ removed. Fig. 7. Branching ratio, $R_{21}=\sigma_{n=2} / \sigma_{1 s}$, for the $\mathrm{He}^{+}(\mathrm{n}=2)$ satellite relative to the $1 \mathrm{~s}$ main $l i n e$ in the resonance region below the $n=3$ threshold. The solid and dashed curves are fits to the data with and without monochromator broadening, 
respectively, as described in the text.

Fig. 8. Asymmetry parameter of the $\mathrm{He}^{+}(\mathrm{n}=2)$ satellite in the resonance region below the $n=3$ threshold. The solid curve is

a fit to the data using the form of Eq. (15). The dashed curve is the same fit with the monochromator broadening removed by the method described in the text. 


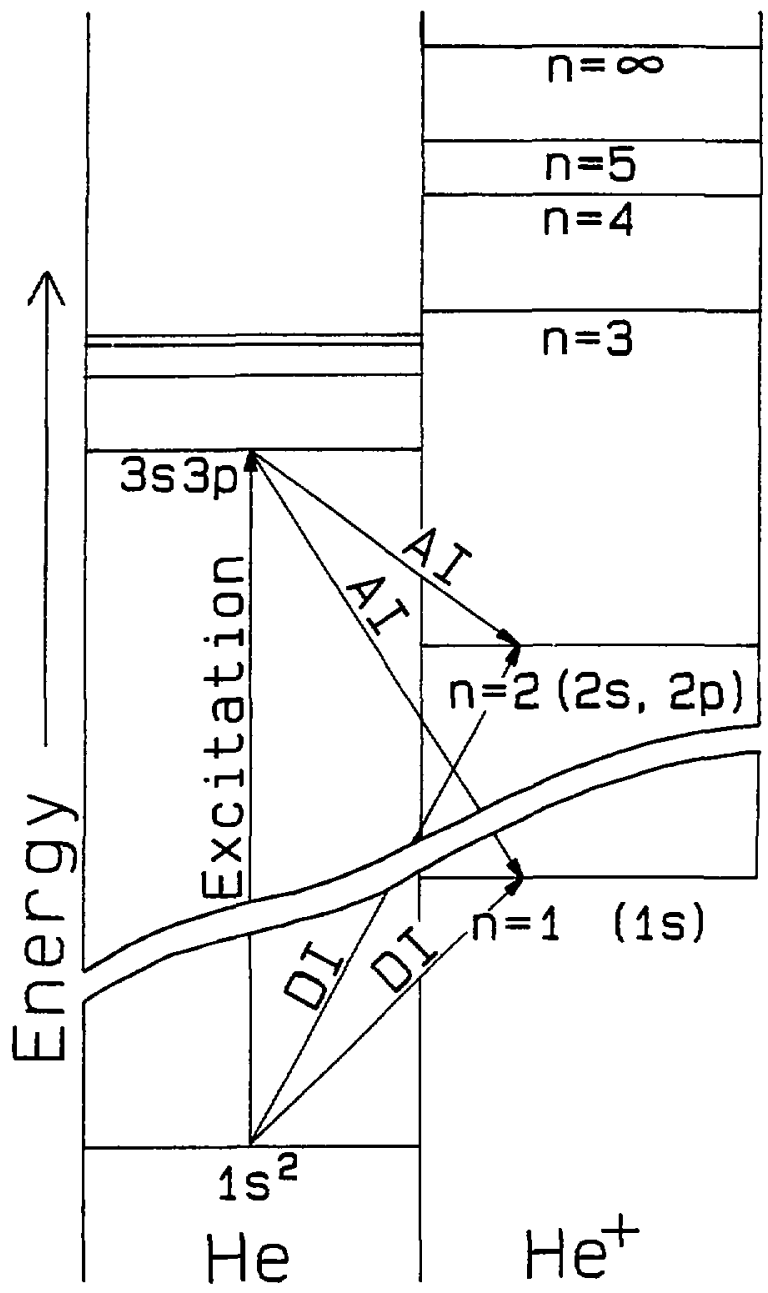

XBL $834-9460$

Figure 1 


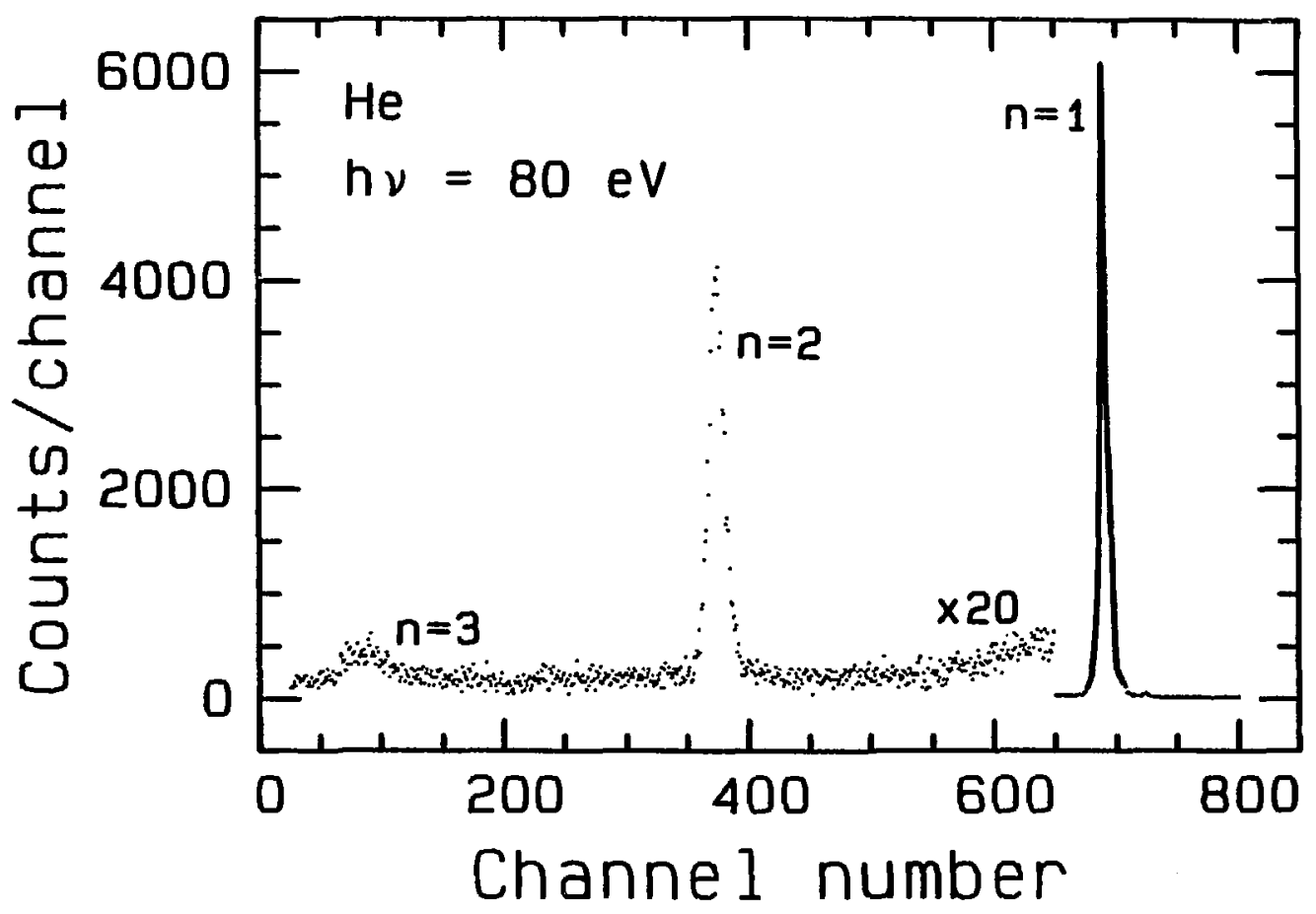




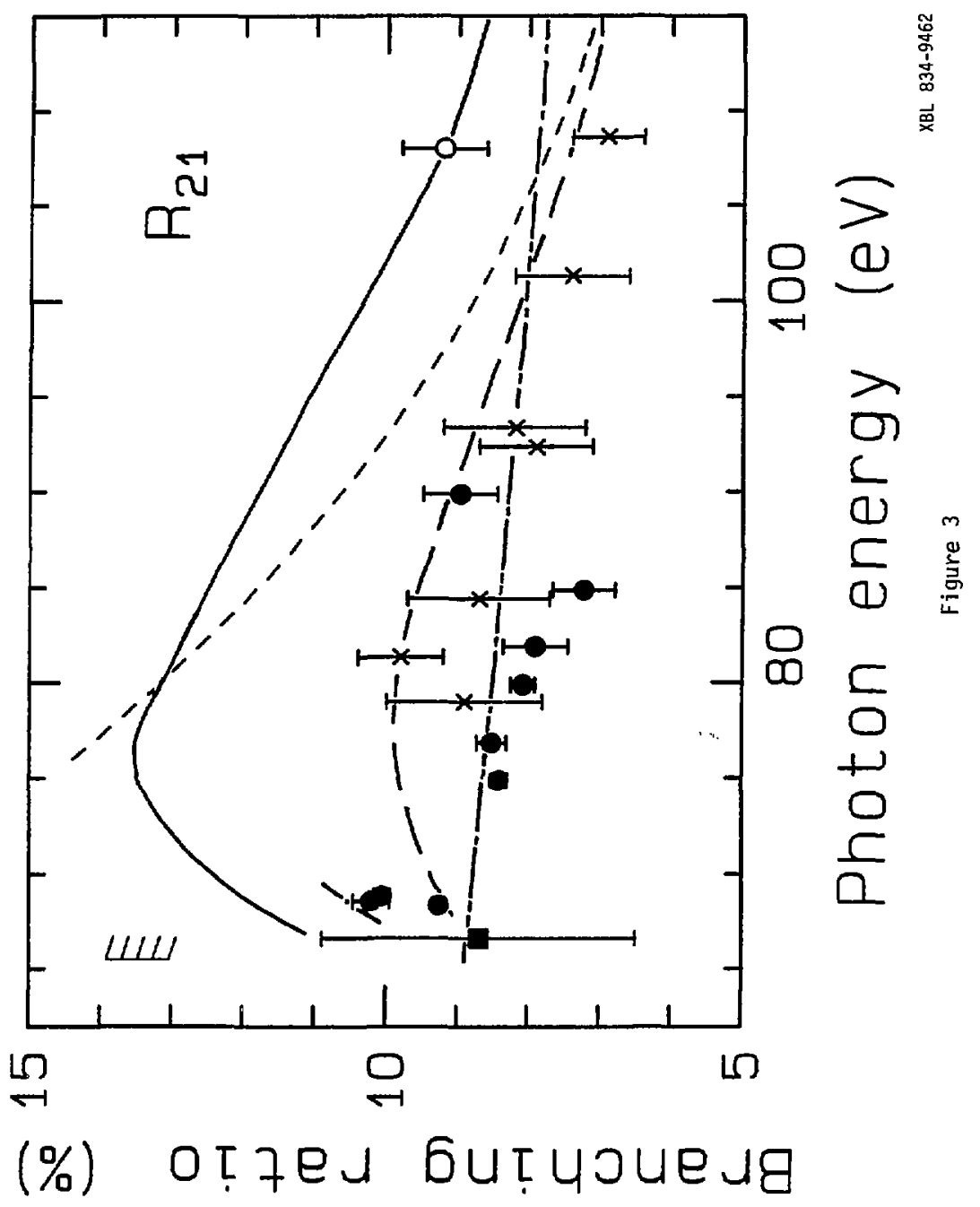




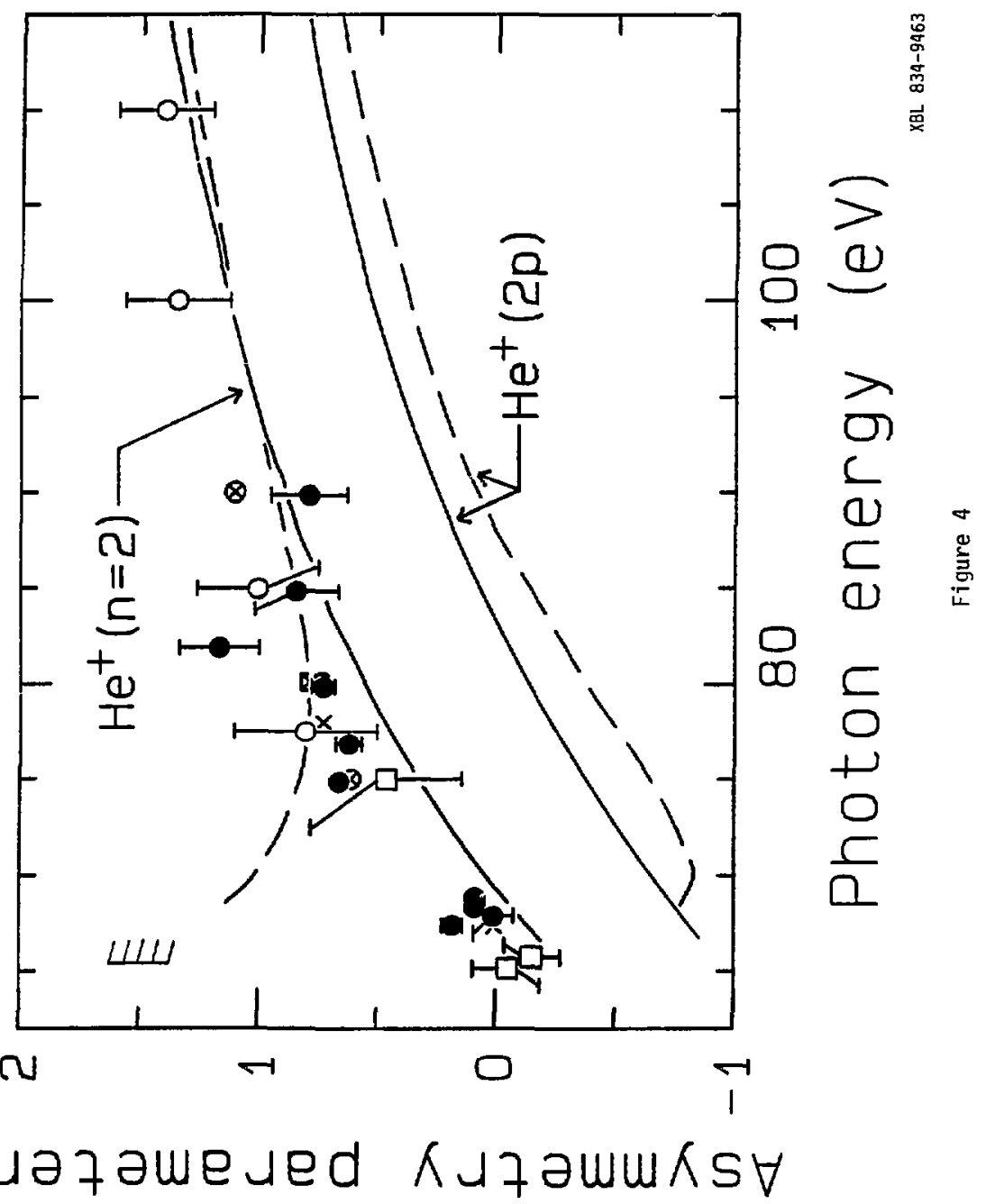




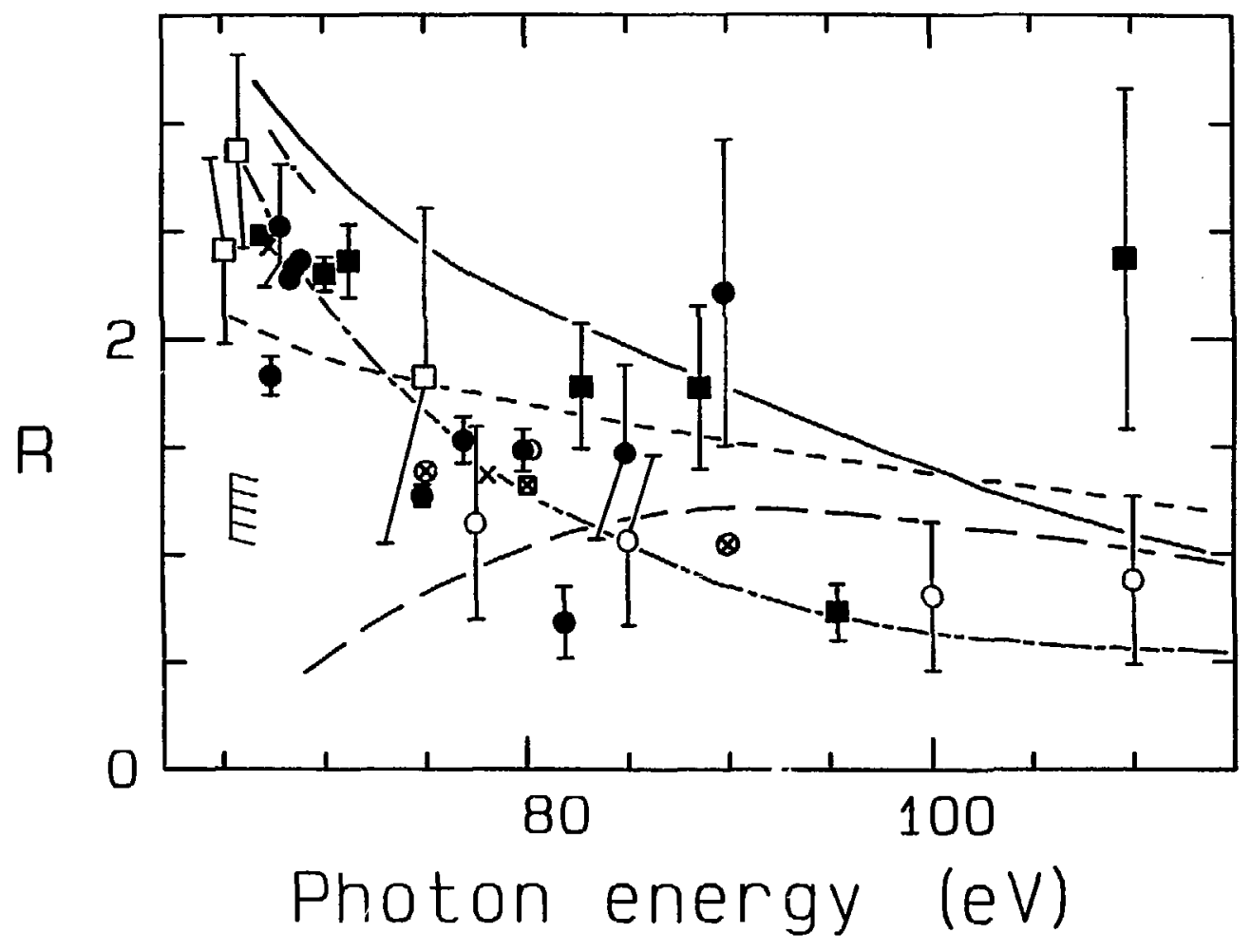

Figure 5 


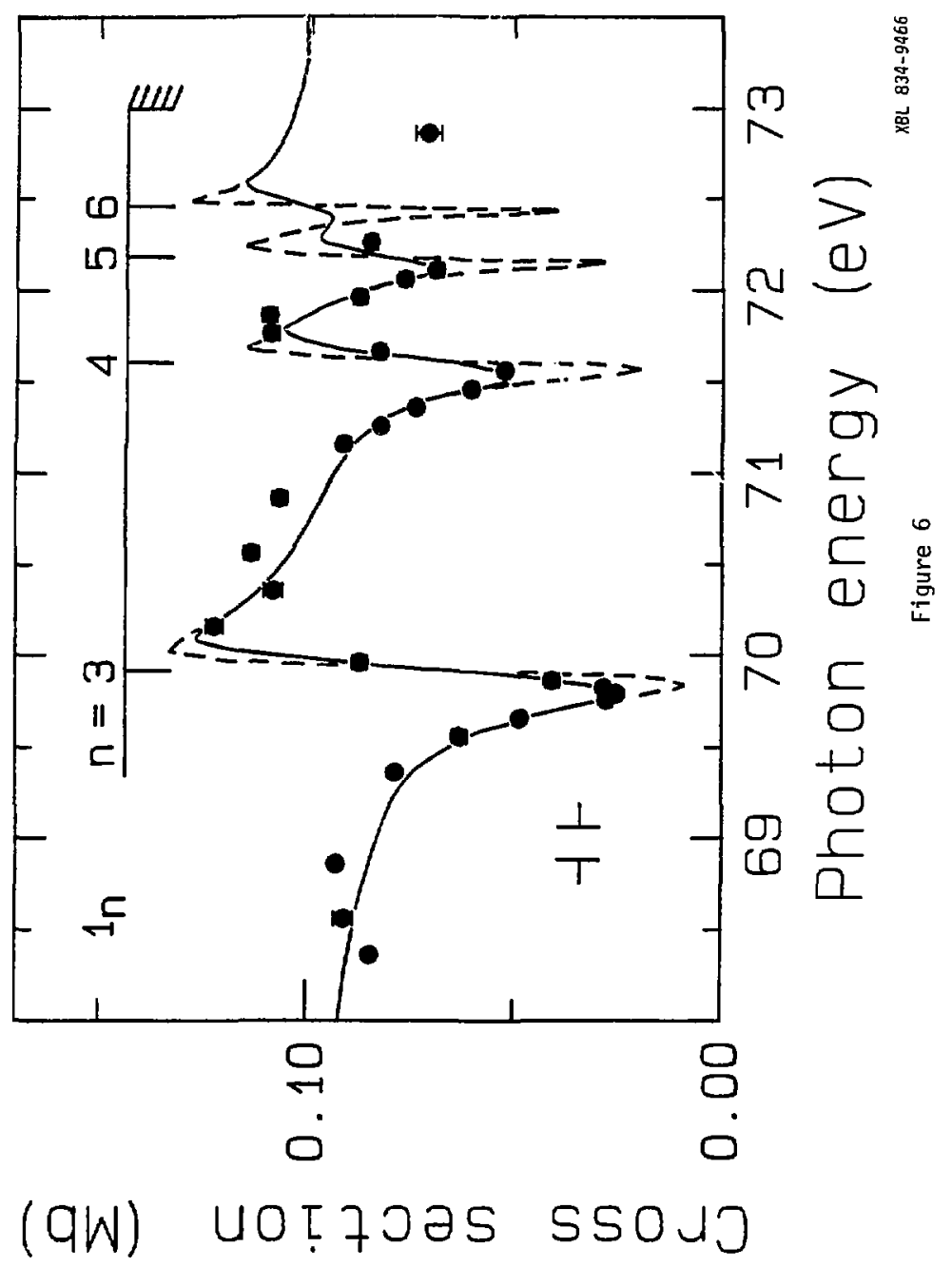




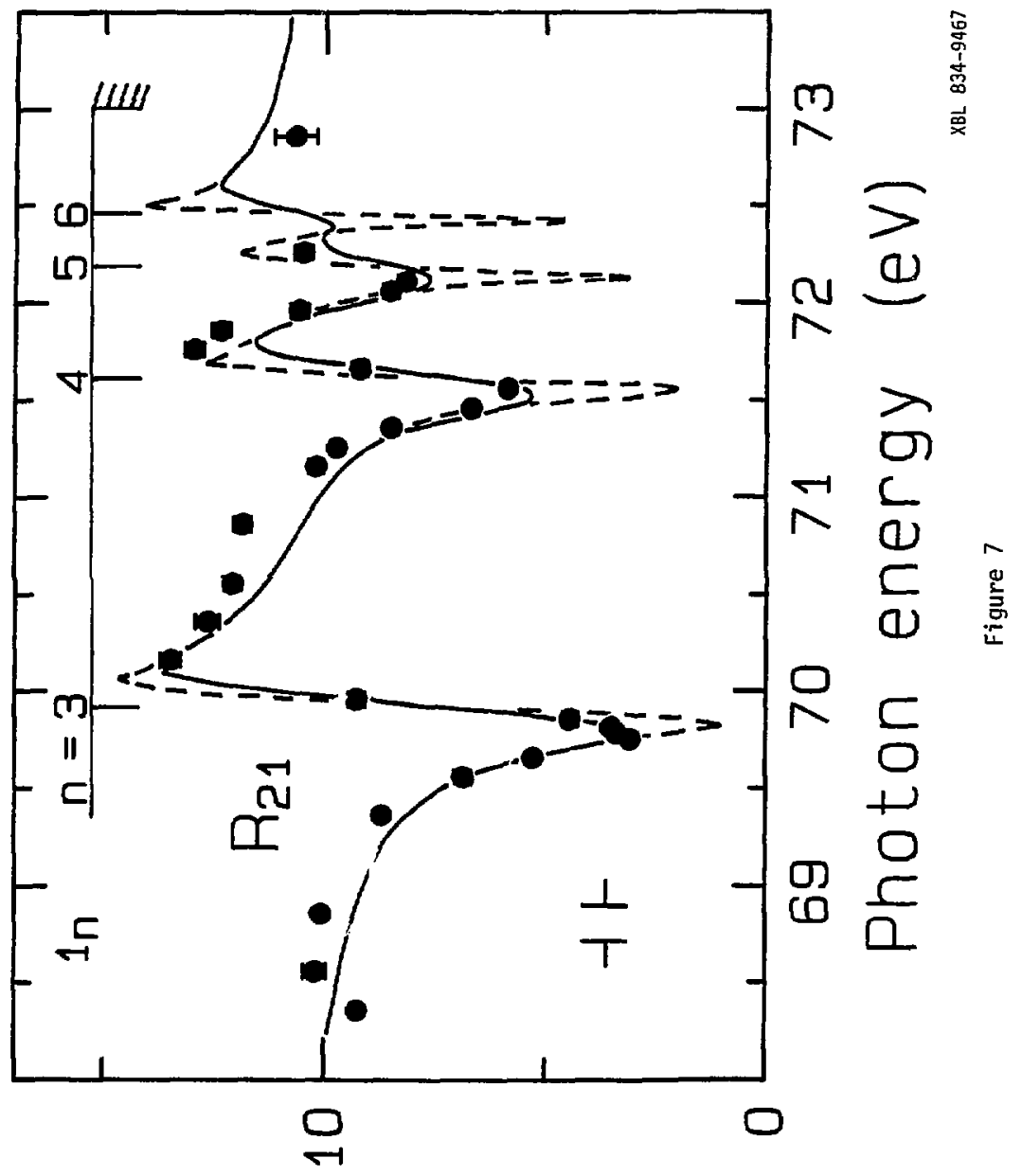

(\%) otgen buțuneug 


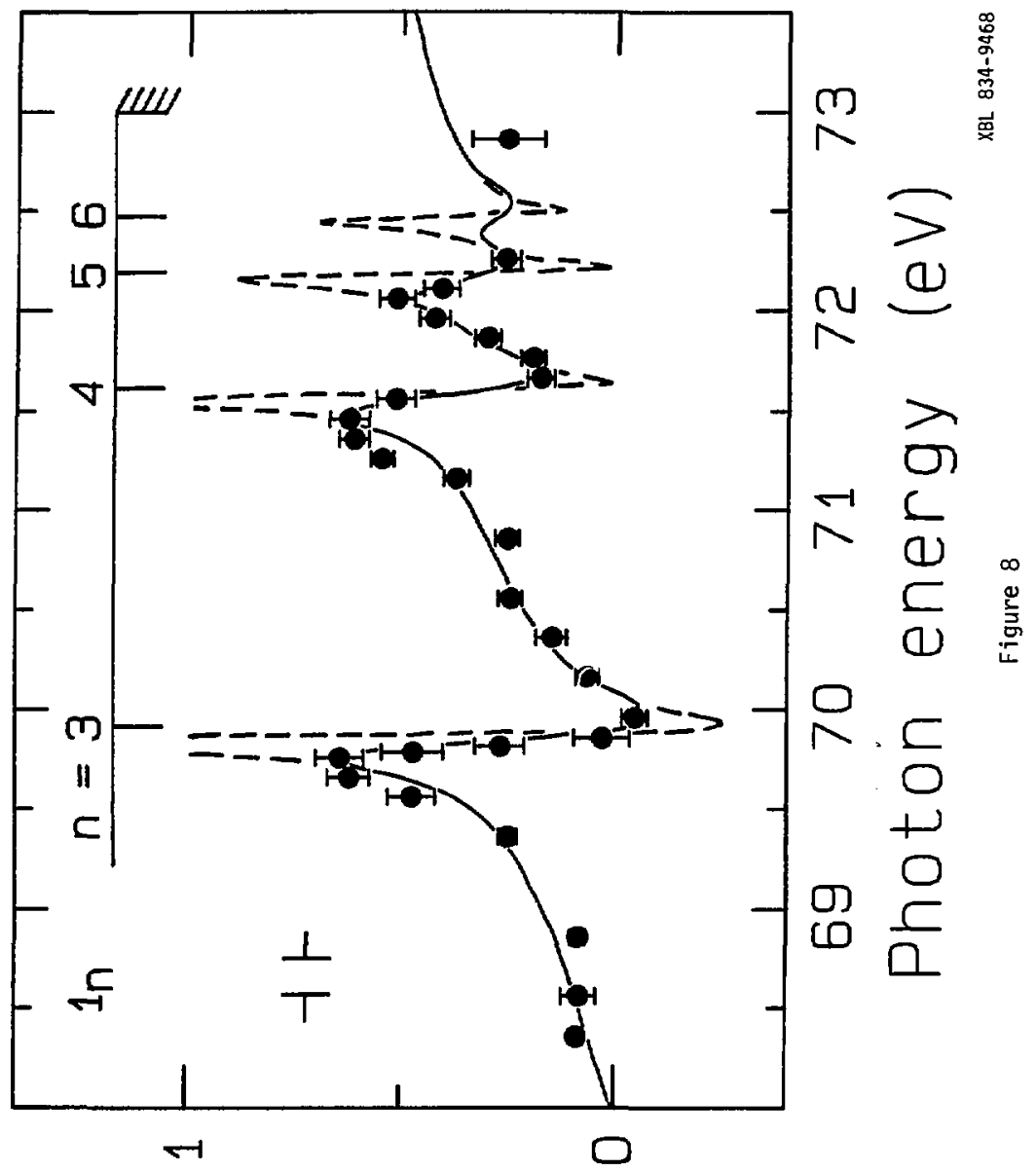

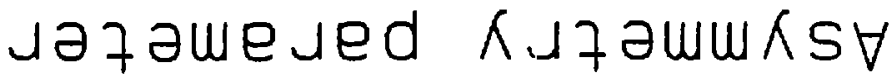




\section{ACKNOWLEDGEMENTS}

I wish to thank my research director, Dave Shirley, for providing a conducive atmosphere within which to pursue my interests. His scientific insight and willingness to allow me to follow my own path were especially appreciated.

All of the work in this dissertation was a collaborative effort with several people. The most significant contributions were provided by Steve Southworth, Paul Kobrin, and Carlton 'Trues' Truesdale; my colleagues for most of my time at Berkeley. Valuable assistance also has been given by Trish Ferrett, Phil Heimann, Uwe Becker, Bill Brewer, Hans Kerkhoff, and Shige Owaki. Special thanks to Paul Kobrin for making those long shifts at SSRL a little less unbearable.

I also benefited from the experience of Dennis Trevor and Jim Pollard, older students who always had the answers. The remainder of the Shirley group, especially Wini Heppler and Barbara Moriguchi, also has been helpful.

My parents and family have been very supportive throughout my graduate career. Their encouragement, caring and love has helped to keep me going. I especially want to thank Steve, Carolyn, Ron, Maxine, Loren, and Trish; my closest friends during the last 5 years. 
This repor was done with support from the Department of Energy. Any conclusions or opinions expressed in this repor represent solely those of the author(s) and not necessarily those of The Regents of the University of California, the Lawrence Berkeley Labosatory or the Department of Energy.

Reference to a company or product name does not imply approval or recommendation of the producl by the University of Califormia or the U.S. Department of Energy to the exclusion of others that may be suitable. 